\title{
ANALYSING IMPACT OF SENSOR COUPLING ON MEASUREMENT REPRESENTATIVENESS OF WALL SURFACE TEMPERATURE
}

\author{
by \\ Sadaf Mansour \\ Bachelor of Architecture, \\ Ryerson University, 2012 \\ A Major Research Project \\ Presented to Ryerson University \\ in partial fulfillment of the \\ requirements for the degree of \\ Master of Building Science \\ in the program of \\ Building Science \\ Toronto, Ontario, Canada, 2017
}

CSadaf Mansour, 2017 


\section{Declaration}

I hereby declare that I am the sole author of this MRP. This is a true copy of the MRP, including any required final revisions.

I authorize Ryerson University to lend this MRP to other institutions or individuals for the purpose of scholarly research.

I further authorize Ryerson University to reproduce this MRP by photocopying or by other means, in total or in part, at the request of other institutions or individuals for the purpose of scholarly research.

I understand that my MRP may be made electronically available to the public. 


\section{Abstract}

Analyzing Impact of Sensor Coupling on Measurement Representativeness of Wall Surface Temperature.

Master of Building Science,2017

Sadaf Mansour

Building Science

Ryerson University

Poor thermal-coupling between sensor and surface is one of the most important factors causing inaccuracy in measurement. Different methods had been suggested by scientists to solve this issue. Embedding the sensor into an object was one of these methods. The goal of these simulations was to assess the impact of sensor coupling on measurement representativeness of wall surface temperature. For this purpose, a cylindrically-shaped sensor was embedded into the internal surface of the wall assembly. The levels of tightness varied from $10 \%$ to $90 \%$, which corresponded respectively from very loose to very tight conditions. Also, in this process the impact of other factors such size and materiality of the sensors' accuracy were evaluated and discussed briefly.

In this study, the results proved that as the sensor decoupled more from the surrounding environment, more accurate data was generated from it. Also, the results from the simulations signified the importance of the temperature difference between the wall surface and the indoor air temperature. The temperature 
difference had a direct relationship with sensor accuracy and measurement representativeness, where smaller temperature difference was associated with higher accuracy. 


\section{Acknowledgments}

I would like to express my sincere gratitude and deep regards to my supervisor, Dr. Zaiyi Liao, and my second reader, Dr. Ramani Ramakrishnan for their constant motivation, monitoring and guidance throughout my research project. In fact, this is a new beginning for professionals like us to extend technology beyond boundaries in the building science industry.

My prime motivation behind this, is my loving family, Mom, Dad and specially my little sister, Mahtab, this journey without their support would have been impossible. 


\section{Contents}

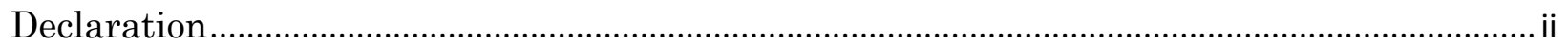

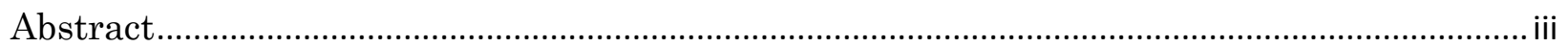

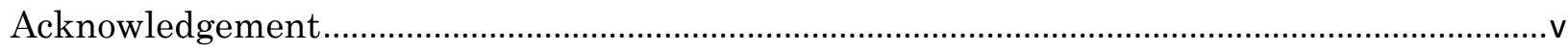

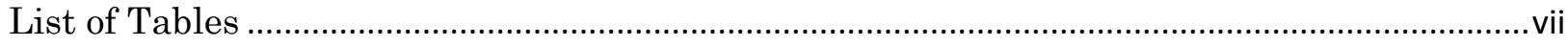

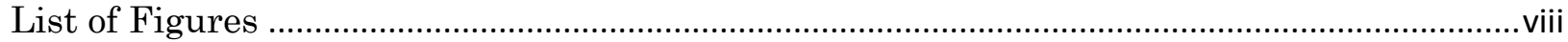

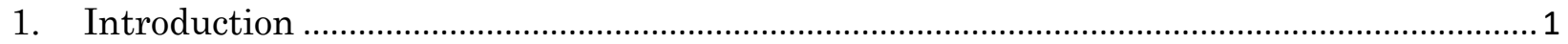

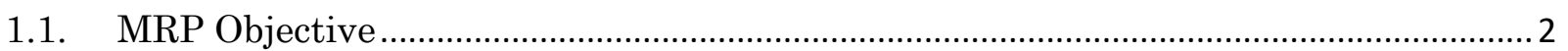

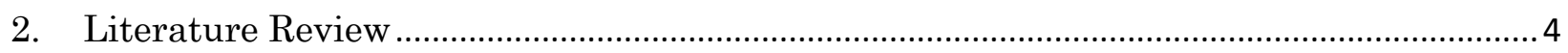

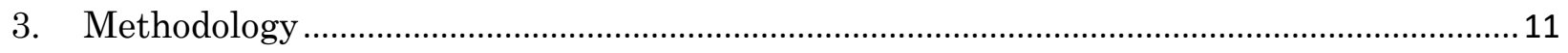

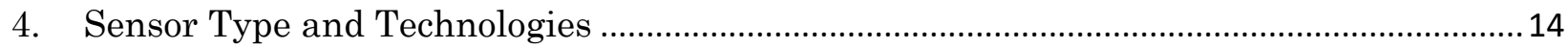

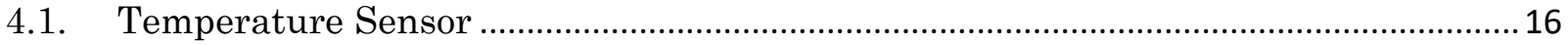

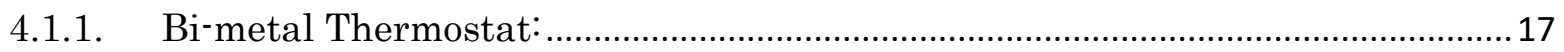

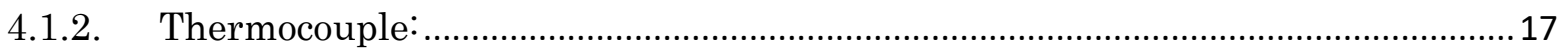

4.1.3. Resistance Temperature Detectors (RTD) ...................................................... 18

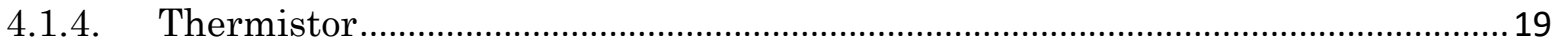

4.1.5. Advantages and Disadvantages of NTC vs. Thermocouple \& RTDs..................20

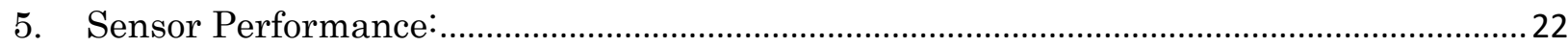

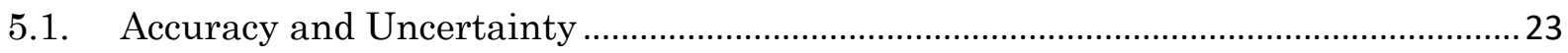

5.2. Equilibrium and Thermal Coupling .................................................................. 23

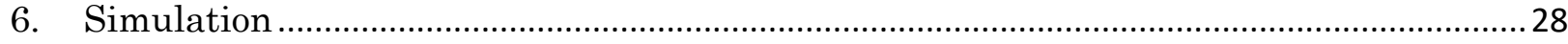

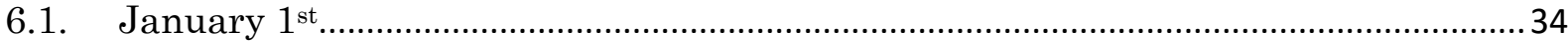

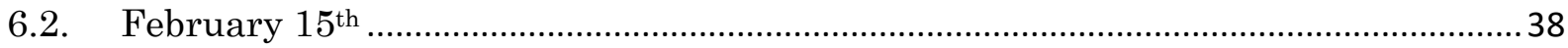

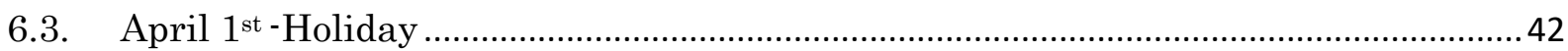

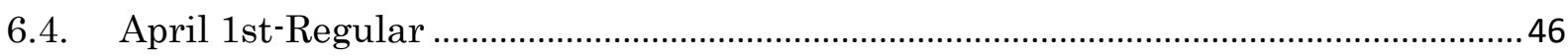

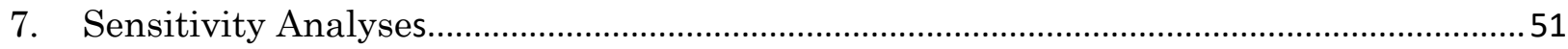

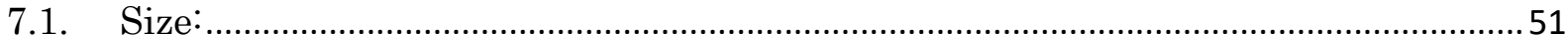

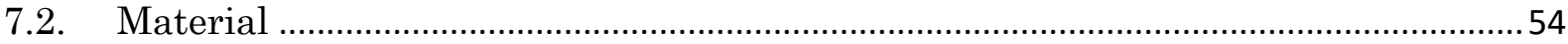

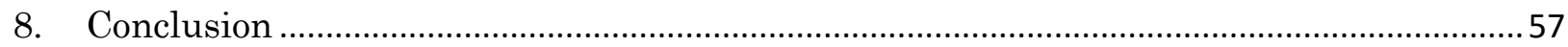

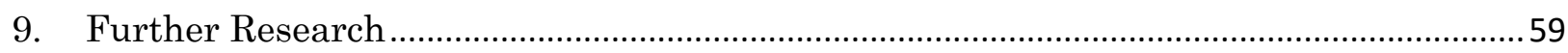

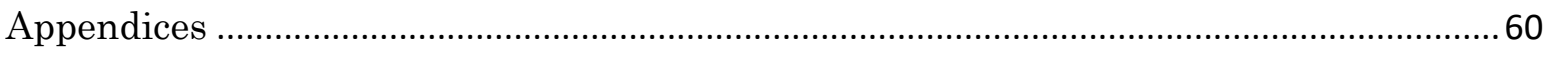

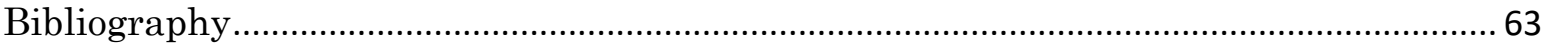




\section{List of Tables}

Table 1

Table 2

Table 3-Sensor's classification (refer to Appendix 1)

Table 4

Table 5

Table 6- Comparison of popular sensor metals

Table 7 36

Table 8

Table 9. 45

Table 10 48

Table 11 50

Table 12

Table 13 53

Table 14. 55

Table 15 .56 


\section{List of Figures}

Figure 1- Radiation can change indoor surface temperature ........................................2 Figure 2- Imbedded temperature sensor (a) and surface temperature sensor with an active

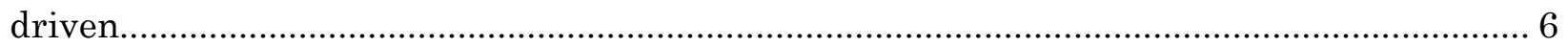

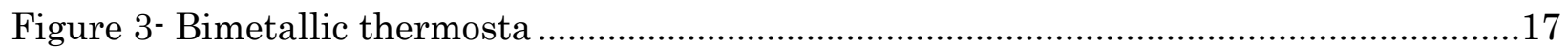

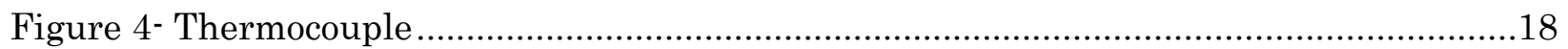

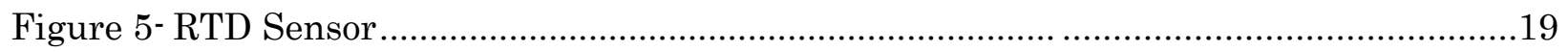

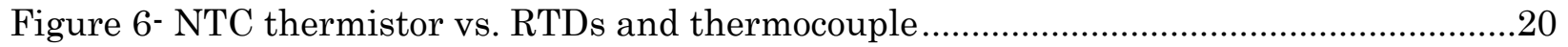

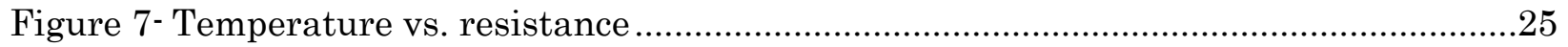

Figure 8- Resistance between sensor, environment and object. ....................................26

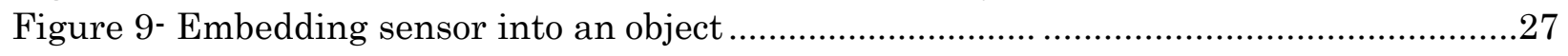

Figure 10- Simulink construction detail.................................................................,29

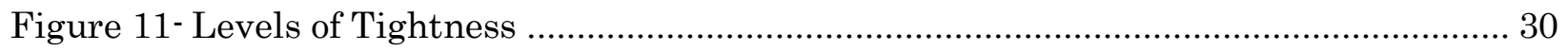

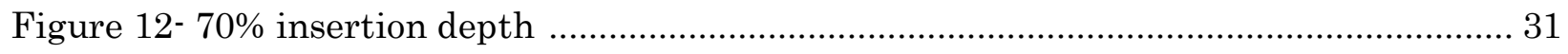

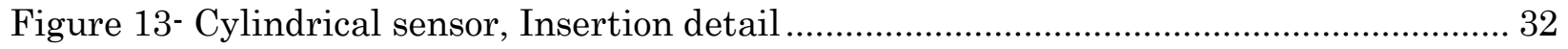

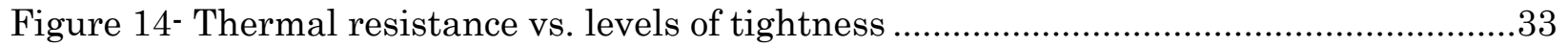

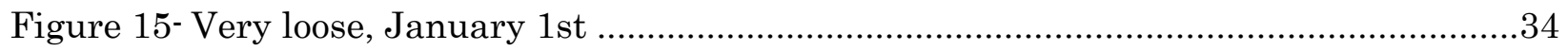

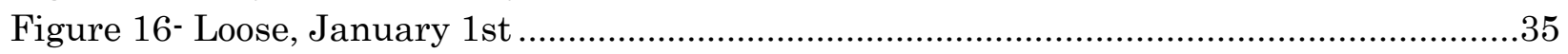

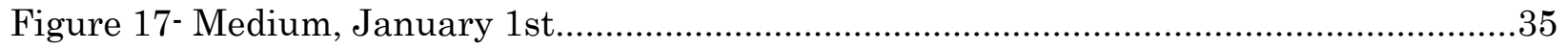

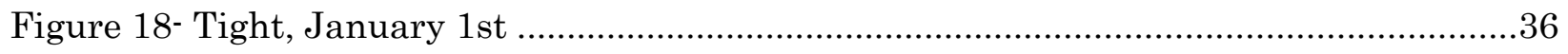

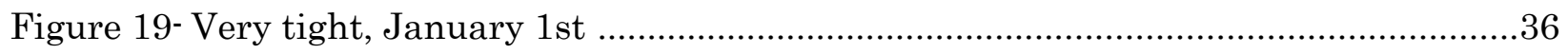

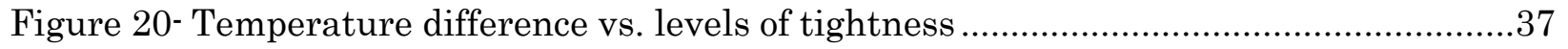

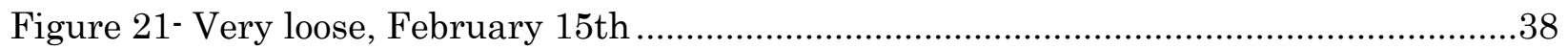

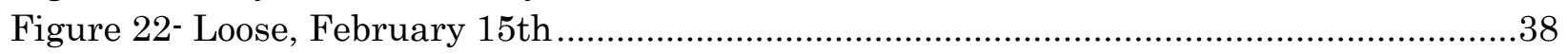

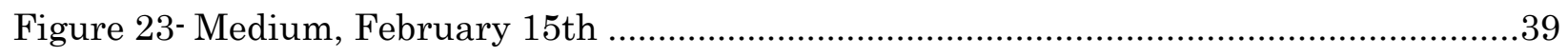

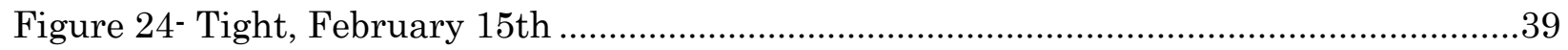

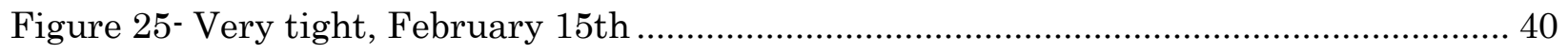

Figure 26- Temperature difference between sensor and surface. Jan. $1^{\text {st }}$ vs. Feb. $15^{\text {th }} \quad \ldots . . .41$

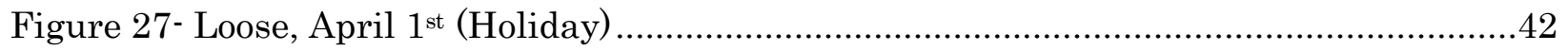

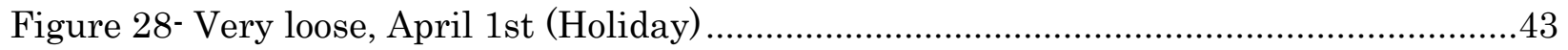

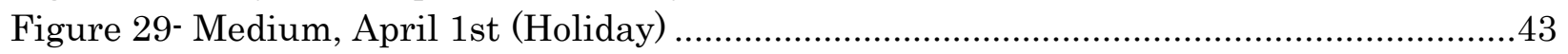

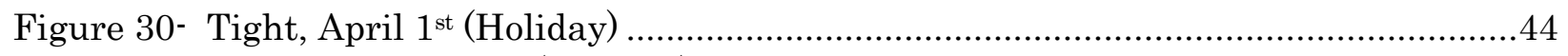

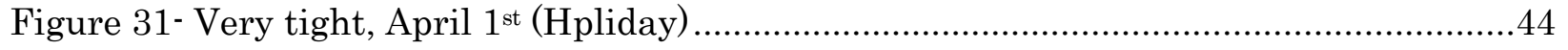

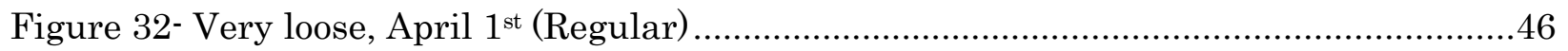

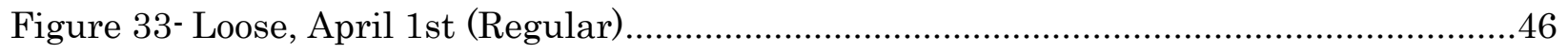

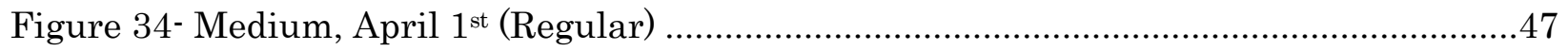

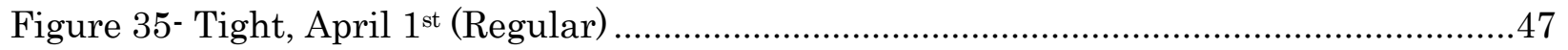

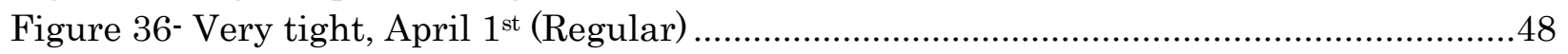

Figure 37- Comparison between Jan. 1 ${ }^{\text {st }}$ Feb. 15 , April 1 $1^{\text {st }}$ Holiday and Regular ...............49

Figure 38 - Size optimization, January 1st ...................................................................... 52

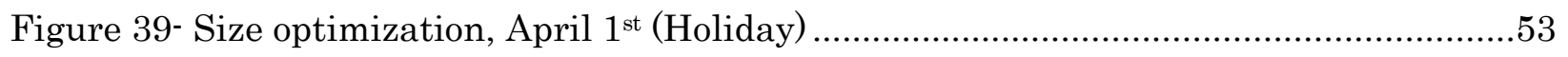

Figure 40- Material optimization, January 1st ..........................................................54

Figure 41-Material optimization- April 1 ${ }^{\text {st }}$ (Holiday) ....................................................55 


\section{Introduction}

This major research project focussed on measurement accuracy of interior wall surface temperature. This type of measurement is not entirely beneficial by itself but its study can be important for future scientific research and industrial uses. However, due to the many extraneous factors, it is often very difficult to obtain measurement results representative enough for the intended purpose. Following are two factors impacting the accuracy of the measurement:

1. Installing a sensor on the surface will distract the temperature distribution on the wall surface, no matter how small the sensor is.

2. The sensor is partially attached to the surface and partially exposed to the ambient air, so the sensor output does not only reflect the surface temperature.

In this study, the assumption was that the surface was homogenous, meaning that installing the sensor on the surface does not distract the temperature distribution. While the aim of this research project was to investigate how the second factor above, the poor thermal-coupling, impacted the measurement accuracy.

Analysing the internal surface temperature of an envelope system was beneficial for measuring the occupant thermal comfort relationship, as well as identifying defects such as thermal bridges and wall assembly deficiencies (improper insulation installation, low insulation R-value, insulation deterioration, etc.). For this purpose, temperature sensor was introduced in this study. Sensors can increase efficiency, accuracy, and speed of measurement systems. Several studies have been carried-out to evaluate the different methods and levels of accuracy and representativeness of indoor air temperature. One of these methods was installing several sensors in different parts of the room and averaging 
indoor air-temperatures (Figure 1). However, this measurement will not be hundred percent accurate and installing several sensors will not resolve this issue and be practical. This research project focussed on measuring wall surface temperatures by applying different levels of tightness between a sensor and the wall surface.

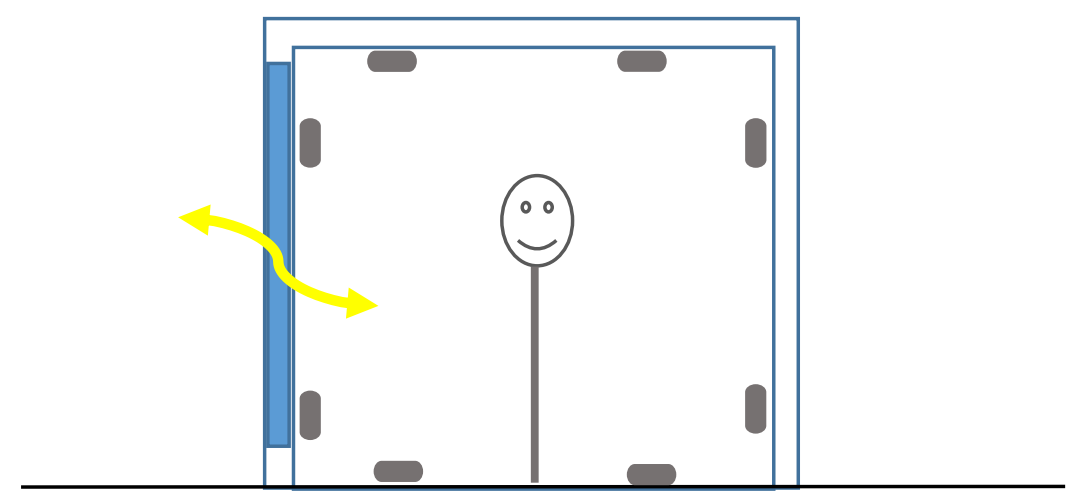

Figure 1- Radiation can change interior wall surface temperature and impact on occupant thermal comfort.

\subsection{MRP Objective}

Different external and internal factors impact on sensors accuracy and "measurement representativeness". The measurement representativeness refers to discrepancy between temperature shown by sensor and actual wall surface temperature.

$$
\Delta T_{\text {Surface }}=\left[T_{\text {actual surface }}-T_{\text {measured surface }}\right]
$$

In reality, the temperature shown by sensor is not 100 percent identical as the wall surface temperature. Other factors such as room air temperature (ambient air) influence on the accuracy.

$$
\left(T_{\text {Sensor }} \neq T_{\text {Wall surface }}\right)
$$

Therefore, a system is needed to accurately monitor indoor air temperature and wall surface temperature to control both energy efficiency and the indoor thermal quality of 
built environment. As discussed, the focus of this project is providing representative measurement of wall surface temperature based on different level of tightness. In this path understanding of different types of thermal sensors and their performance, optimisation methods as well as investigating the factors that influence on sensor's accuracy is essential. 


\section{Literature review}

As it was explained earlier the sensor technology is getting more popular. As the result in the last two decades many studies done in this field. The goal of most of these researches were improving the measurement representativeness, minimising data uncertainty, maximising measurement accuracy and increasing sensor response time. One of the practical subject in this field is measuring the surface temperature. In order to evaluate the surface temperature and its representativeness, it is important to understand the existing weaknesses and drawbacks of temperature sensor. In this literature review the first few articles and books focus on the understanding of temperature sensor, performance and area of inaccuracy while the last few article focus on measurement representativeness and mathematical method.

In a research conducted by David Culbertson in 2001, he had studied various contacted type surface temperature sensor and he pointed out at several drawbacks associated with them. One of the weaknesses in all these sensors was at the thermocouple junction. The junction must be in direct contact with the surface to be able to measure temperature accurately. In this research, Culbertson indicated that improper lap joints, where one of the thermocouple material overlap the other, prevents junction from uniformly contacting the surface. This ununiform contact result in substantial thermal resistance and as the consequence errors introduce in to the system.

Also, he introduced the electrical insulating layer between the thermocouple junction and the junction holding apparatus as another weakness in most of contact type surface temperature. The insulating layer which is electrically insulated, contains a substantial thermal mass. This mass provides a thermal loading effect on the junction. All this process cause measurement error in system and a slowing down the response time. For fixing this 
weakness, junction requires undesirably long period of contact with the surface to provide accurate thermal measurement.

In another study done by Richard Whit in 1987, he investigated and analysed different types of sensors. The focus of this research paper was providing a practical way to comparing sensors and obtaining a comprehensive overview of them. White divided sensors in to three categories, physical, chemical and biological. Also, he finely subdivided hierarchical categories used by abstracting journals. The sensor classification in this research include six tables.

1. Table I list alphabetically most measurands for which sensors

2. Table II is focused on technical aspect. Primarily sensor characteristic such as sensitivity, selectivity and speed of response.

3. Table III is focused on detection means used in sensors

4. Table IV is interested primarily to technologists involve in sensor design

5. $\mathrm{V}$ is related to materiality and fabrication

6. Table VI is sensor field of application

One of the valuable source for analysing sensor's accuracy performance is handbook of modern sensor by Jacob Fraden, 2010. In the chapter sixteen of this book he focused on temperature sensors. He introduced poor coupling as one of the main reason of inaccuracy in result. "Any sensor, no matter how small, will disturb the measurement site and thus cause some error in temperature measurement. Thus, it is an engineering task to minimize the error by an appropriate sensor design and a correct measurement technique of which the coupling between the sensor and object is most critical" (Fraden, 2010). 
In this study, he tries to prove the importance of coupling and size of the sensor in thermal equilibrium by using mathematical model. He claims that the size of sensor has direct relationship with speed of response time. The smaller the sensor the faster the response time. Also, in another part of the research he used mathematical model to investigate the relationship between poor thermal coupling and thermal resistance. "If a sensor is coupled not only to the object whose temperature is measured, but to some other items as well, an error is introduced" (Fraden, 2010). "After the moment of coupling or after the object temperature changes, the sensor's temperature at any moment of time is $T_{\text {Sensor }}$, while the object has true temperature $T_{\text {object }}$. Almost never these two temperatures are equal. The goal of the equilibrium measurement is to bring $T_{\text {Sensor }}$ as close to $T_{\text {object }}$ as possible" (Fraden, 2010).

He suggests that the best way to decouple the sensor from environment is embedding the sensor into the object. Also, he was added to fill the cavity preferably with a thermal grease, epoxy, or other method and use the thermally bond the sensor with the cavity walls but as it is obvious having cavity inside the wall is not always practical. In this case using
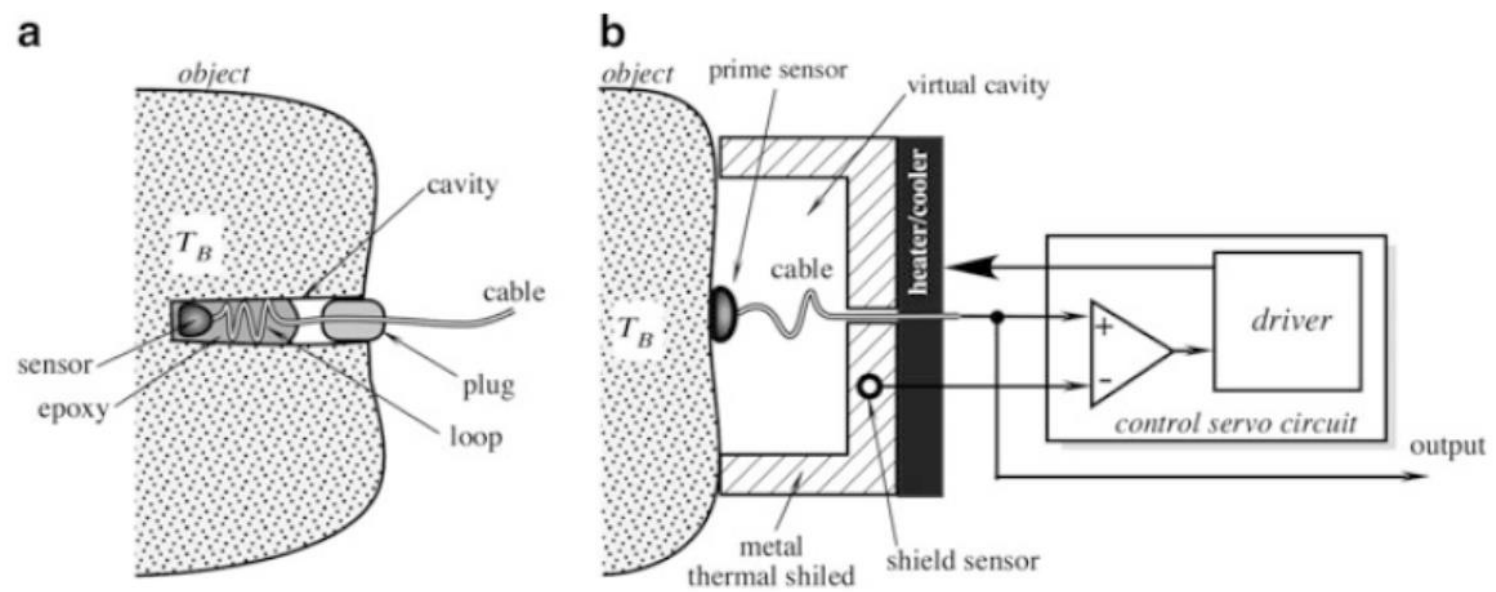

Figure 2- (a) Imbedded temperature sensor in to the object, (b) surface temperature sensor covered up with a shield (Fraden, 2010) 
protective envelope was suggested. this protection can be either thermal cover or glass shield or any other material with high thermal conductivity.

Also, another study conducted by Edward Coy in 2010. He focused on the same topic, the benefits of embedding sensor into the object. In this research study, he was used experimental model to prove effectiveness of this method on measurement of heat flux. Also, he analysed the accuracy of the results by using two sensors, one embedded into the object and one at the surface. He strongly believed on this quote: "Do not relying on surface temperature measurements but embed the sensors within the wall where they are protected from erosion" (Coy, 2010). Also, he suggests using more well calibrated sensor as these devices can control each other's performance and generate more accurate result.

As noted above many scientists such as, Farden and Coy came up with different solutions to minimise uncertainty and increase measurement representativeness on the surface temperature measurement. Hiraka et al. in 2008, studied contacted and non-contacted temperature sensor as well as advantages and disadvantages of each category. Table 1 is showing these in detail. In this process Hiraka et al. came up with the new idea of hybridtype thermal sensor which combines advantages of both system. "This system is composed of two main components: a metal film that contacts the object and an optical sensor which is used to detect the radiance of rear surface of metal film. If the emissivity of rear surface of the film is known in advance, the true temperature of the metal film can be derived from the radiance signal detected by the optical sensor after correcting for the known emissivity of rear surface of the film. Under a thermally steady condition between the object and metal film, the true surface temperature of the object can be ascertained from the temperature reading of the rear surface of metal film, in spite of a possible emissivity change of measure object" (Hiraka, Shinagawa, Gogami, \& Luchi, 2008). 
Comparison of contacted and non-contacted sensor

advantages disadvantages

Contacted sensor stable Cannot be applied to moving object

measurement

Can be deteriorated by time

Non-contacted Rapid response If the emissivity of object changes the radiation

sensor thermometry can no longer accurately measure the temperature of the object

Table 1

Another focus of this study was materiality and sensor fabrication. He strongly suggested silicon material in this invention as it is a good semiconductor and its response time is short.

Pavel Ripka and Alois Tipek are two other scientists that investigated thermal sensors feature and reliability in 2007. Chapter eight of modern sensor handbook is specified to temperature sensor. Ripka introduced the position of sensor, depth of insertion, thickness of the wall of sensor and insulation as main feature of designing a practical sensor. In this book, several subjects were studied such as relationship of voltage and temperature as well as resistance and temperature. As the temperature increase the voltage difference in sensor will rise too but the amount of voltage has direct relation with material used. In other hand with rising temperature in most type of thermal sensor resistance decrease. The amount resistance change is directly related to material used and level of tightness.

In another study conducted by Jon S. Wilson in 2005, he studied different types and technologies of temperature sensor and analysed advantages and disadvantages of each individual type. Also, he explains the application of each type. He introduces Platinum, Nickle, silver and copper as most popular metal used for RTD sensor design and compare their resistance vs temperature. Moreover, he emphasises the importance of calibration in measurement. "calibration can improve the accuracy of sensor by 10 times" (Wilson, 2005). 
As discussed above, most of the literature focused on sensor inaccuracy and method to improve that. While it is important to know about studies done so far to measure the representativeness of data. The following research are explaining the mathematical model in this field and how this method improved energy efficiency and occupant thermal comfort.

In a study conducted by Liao et al. two different temperature prediction model were presented, GA-BP (genetic algorithm back propagation) based model and physical-rule based adaptive neuro-fuzzy inferential sensor (ANFIS) model. The purpose of designing and comparing of these two system were to improve indoor temperature prediction models in the building as well as increasing energy efficiency of district heating. This paper introduces three performance measurement (RMSE, RMS and R2). "Root Mean Square Error (RMSE) which measures the mean deviation (error) of the predicted values to the measured indoor temperature values. The Maximum Relative Error (MRE) which is a measure for the largest error (or the farthest point) as well as goodness of fit (R2). R2 generally takes values between 0 and 1" (Liao \& Huang, 2012). In the testing period, Physical-Rule based prediction model presents a better performance than the other model.

In another study done by Liao et al. the performance of inferential sensor on estimate the average air temperature in multi-zone heating systems was investigated. The success in this experiment helps to improve the overall performance of heating systems in terms of energy efficiency and thermal comfort. In this study both experimental model and an inferential sensor model were developed. neural fuzzy method has been designed based on adaptive neuro-fuzzy inference system modeling which provide estimation of the average air temperature in multi-zone space heating systems. In this study model performance is measured using the root mean-square error (RMSE) and is defined as 


$$
\sqrt{\frac{1}{N} \sum_{i=1}^{N}\left[T_{\text {Observed }}-T_{\text {Predicted }}\right]^{2}}
$$

The average air temperature results estimated by using the developed model are strongly in agreement with the experimental results (Liao, Jassar, \& Zhao, 2009). 


\section{Methodology}

The selected methodology for this research project started by collecting academic literature, journals and articles to build background information. The next step was to provide some of the information by model and formula. The final step was to develop a computer model and simulation. For this purpose, Simulink which is a graphical programming mathematical environment for modeling, was selected. The simulation includes two parts:

- Assumption and settings

- Measurement method

\subsection{Assumption and settings}

The followings are the key design informations of the Simulink model:

\section{Existing design:}

A 3-bedroom house with a kitchen and a living room. The bedroom-1 is the focus of this project. This room was designed with an exterior wall. The wall assembly was designed based on OBC requirements which included finish stucco, EPS, gypsum board, wood stud framing and plaster. Also, as the simulation was done during the cold season, the room was designed with a heating radiator and a controller system. The controller system is responsible for adjusting temperature based on occupancy hours.

\section{Sensor Design:}

Designing the sensor was the most crucial part of this project. The proposed sensor is cylinder shape, $1 \mathrm{~mm}$ radius and $5 \mathrm{~mm}$ height. The selected material is copper with heat capacity of $0.385, / g *{ }^{\circ} \mathrm{C}$. The sensor was installed vertically in the middle height of 
internal wall surface and the level of tightness between sensor and wall vary from $10 \%$ to 90\% (very loose to very tight). The sensor generated data based on two variables, actual wall surface temperature and indoor air temperature (ambient air).

\section{Occupancy schedule:}

The occupancy behaviour plays an important role in this simulation as occupants leave the house between 9 am to $6 \mathrm{pm}$. This period is called setback hours which heating radiator works at minimum. Two hours prior to occupancy hour radiator starts to warm up. The indoor air temperature sets between $18^{\circ} \mathrm{C}$ and $23^{\circ} \mathrm{C}$ (comfort range). Also, the simulation model was programmed to have some pre-set days as holidays (which there are no occupancy at room during those days) in which the heating radiator works at minimum constantly.

\section{Simulation days and period:}

Three different days were selected for analysing the impact of sensor's tightness on performance of the system. Based on Canadian climate data January is the coldest month of the year and February is the second one. Therefore, three dates of January $1^{\text {st }}$, February $15^{\text {th }}$ and April $1^{\text {st }}$ were chosen to represent very cold, cold and mild temperature, respectively. The goal of selecting different days was to evaluate the system performance on different weather condition. The length of each simulation period was 24 hours.

\subsection{Measurement method}

Based on the academic literatures the suitable measurement method for these types of projects is "Root Mean Square Error" (RMSE) method which measures the mean 
deviation (error) of the predicted values (sensor's data) to the expected values (actual wall surface temperature).

$$
\text { Temperature Error (sensor and surface) }=\sqrt{\frac{1}{\mathrm{~N}} \sum_{\mathrm{i}=1}^{\mathrm{N}}\left[\mathrm{T}_{\text {predicted (sensor) }}-\mathrm{T}_{\text {Expected (surface) }}\right]^{2}}
$$

In this project the RMSE method generates two sets of data, column A and B (Table 2). the column A indicates the temperature difference between the sensor and the surface. As it is shown on the example chart, with increasing the level of tightness, RMSE gets smaller. This reduction indicates higher measurement accuracy while on column $\mathrm{B}$, the procedure is completely opposite. In column B with increasing the tightness level between sensor and surface, temperature difference between sensor and air is getting larger. The larger number indicates lower impact of ambient air on sensors accuracy and measurement representativeness. In fact, numbers are fluctuating between 0 and 1 , (high accuracy $0<$ number $\leq 1$ low accuracy)

\begin{tabular}{|c|c|c|c|c|}
\hline \multicolumn{5}{|c|}{ Example Chart } \\
\hline \multirow{7}{*}{ 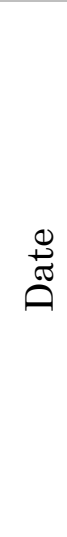 } & $\begin{array}{l}\text { Contact } \\
\text { level }\end{array}$ & $\begin{array}{l}\text { Contact } \\
\text { percentage }\end{array}$ & $\begin{array}{l}\text { Temp. difference between } \\
\text { sensor and air }{ }^{\circ} \mathrm{C}\end{array}$ & $\begin{array}{l}\text { Temp. difference between } \\
\text { sensor and surface }{ }^{\circ} \mathrm{C}\end{array}$ \\
\hline & $\begin{array}{l}\text { between } \\
\text { sensor \& } \\
\text { surface }\end{array}$ & & $\sqrt{\frac{1}{N} \sum_{i=1}^{N}\left[T_{S e n s o r}-T_{A i r}\right]^{2}}$ & $\sqrt{\frac{1}{N} \sum_{i=1}^{N}\left[T_{\text {Sensor }}-T_{\text {Surface }}\right]^{2}}$ \\
\hline & Very Loose & 90 & 0.27 & 0.59 \\
\hline & Loose & 70 & 0.56 & 0.31 \\
\hline & Medium & 50 & 0.71 & 0.17 \\
\hline & Tight & 30 & 0.80 & 0.08 \\
\hline & Very Tight & 10 & 0.86 & 0.02 \\
\hline
\end{tabular}

Table 2 


\section{Sensor type and technologies}

Different types of sensors are existing in market ranging from very simple to complex. The sensor's classification strongly depends on the purpose of use. In general sensors are divided in two categories

- Passive and active: a passive sensor is working independently from any additional energy source and directly generates an electrical signal in response to an external stimulus. While the active sensors require external power for operation. In this scenario, the sensors modify the signal to produce output signals

- Absolute and relative: "An absolute sensor detects a stimulus in reference to an absolute physical scale that is independent of the measurement conditions, whereas a relative sensor produces a signal that relates to some special case" (Fraden, 2010). One of the good example of relative sensor is thermocouple.

- Properties: another method of classifying sensors is based on its properties. several research done in this field and the following tables are dividing different characteristic of sensors (Table 3). 
Table 1.1 Sensor specifications

Table 1.2 Sensor material

Table 1.3 Detection means used in sensors

Table 1.4 Conversion phenomena

\begin{tabular}{ll}
\hline Sensitivity & Stimulus range (span) \\
\hline Stability (short- and long-term) & Resolution \\
Accuracy & Selectivity \\
Speed of response & Environmental conditions \\
Overload characteristics & Linearity \\
Hysteresis & Dead band \\
Operating life & Output format \\
Cost, size, weight & Other \\
\hline
\end{tabular}

\begin{tabular}{ll}
\hline Inorganic & Organic \\
\hline Conductor & Insulator \\
Semiconductor & Liquid gas or plasma \\
Biological substance & Other \\
\hline
\end{tabular}

Biological

Chemical

Electric, magnetic or electromagnetic wave

Heat, temperature

Mechanical displacement or wave

Radioactivity, radiation

Other

\begin{tabular}{ll}
\hline Physical & Thermoelectric \\
Photoelectric & Photomagnetic \\
Magnetoelectric & Electromagnetic \\
Thermoelastic & Electroelastic \\
Thermomagnetic & Thermooptic \\
& Photoelastic \\
& Other \\
& Chemical transformation \\
& Physical transformation \\
& Electrochemical process Spectroscopy \\
Other \\
Biochemical transformation, Physical \\
transformation \\
Effect on test organism Spectroscopy \\
Other
\end{tabular}

Table 3- Sensor's classification (refer to Appendix 1)

(White, 1987) 


\subsection{Temperature sensor}

Temperature have a significant effect on material at molecular level and it defined as a specific degree of coldness or hotness as reference to a specific scale. In fact, heat released from objects is consequence of molecular energy (vibration and friction). The higher heat energy the greater the molecular energy. Temperature sensor detect a change in physical parameter such as resistance or output voltage that corresponds to a temperature change. There are two basic types of temperature sensing:

- Contacted: Contacted temperature sensing requires the sensor to be in direct physical contact with media or object being sensed. It can be used to monitor the temperature of solid, liquid or gases over an extremely wide temperature range (Wilson, 2005)

- Non-contacted: Measurement interprets the radiant energy of a heat source in the form of energy emitted in infrared portion of the electromagnetic spectrum. This method can be used to monitor non-reflective solid and liquid but it is not effective with gases due to their natural transparency (Wilson, 2005).

Temperature sensor is divided to three categories (electro-mechanical, electronic and resistive) each of these families have unique characteristics and they are suitable for specific environmental condition. The following chart illustrates the three families and popular temperature sensors in each category. Also, the technology behind selected of these sensors will be explained further.

\begin{tabular}{|l|l|l|}
\hline \multicolumn{2}{|c|}{ Temperature Sensors } \\
\hline Electro mechanical & Electronic & Resistance device \\
\hline Bi-metal thermostat & Silicon sensors & Thermistor \\
Bulb and capillary & Infrared pyrometry & Resistive temperature \\
thermostat & Thermocouple & device (RTD) \\
\hline
\end{tabular}

Table 4 


\subsubsection{Bi-metal thermostat:}

In this system two metallic strips, with different coefficient of expansion are attached to each other at a base temperature. Any changes in temperature will cause the strip to expand differently. The compressed strip will experience circular bending (Figure 3). By measuring the expansion and contraction and comparing it to normal situation we can measure the temperature differences although tis system is not very accurate. This sensor categorizes as relative and passive.

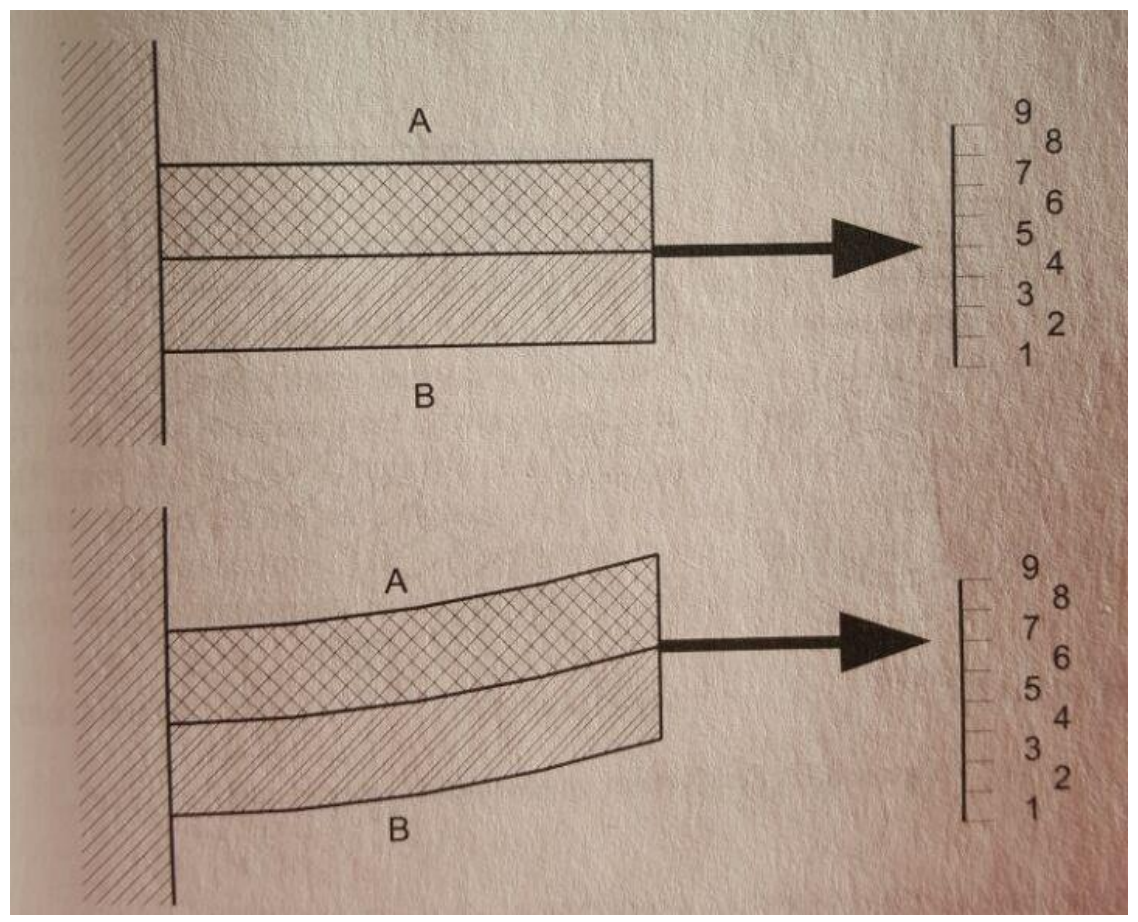

Figure 3- Bimetallic thermostat

Different expansion of metal strip A \& B. (Ripka \& Tipek, 2007)

\subsubsection{Thermocouple}

Thermocouple are basically formed two electrical conductors, alloys or dissimilar metal which are joined at one end of a circuit. One junction is in temperature T1 and the other at temperature T2 (Figure 4). Thermocouple generates a voltage that is proportional to the temperature difference. This action is called Seebeck effect. The voltage generated in this 
performance is depends on the material used and temperature difference between $\mathrm{T} 1$ and T2.

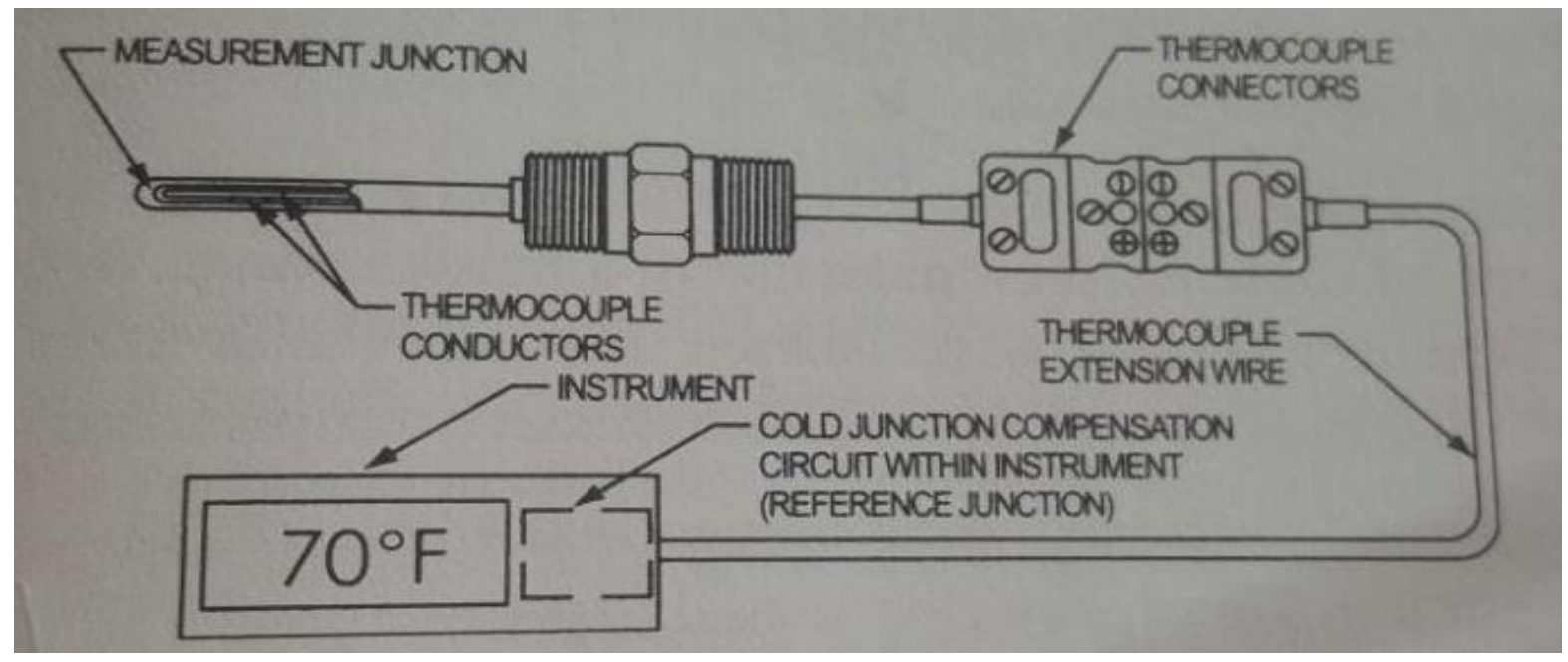

Figure 4- Thermocouple (Wilson, 2005)

All thermocouples have a "hot junction" (measurement) and "cold junction" (reference).

"One end of the conductor (the measurement junction) is exposed to the process temperature and the cold junction can be either a reference junction that is maintained at $0^{\circ} \mathrm{C}$ or electronically compensated meter interface" (Wilson, 2005). One of the advantages of thermocouple over other thermal sensor is the wide temperature range. Also, the key point for keeping accurate result is move the cold joints to a place where the ambient temperature is as stable as possible.

\subsubsection{Resistance temperature detectors (RTD)}

To measure or control temperature, resistive temperature device employ a change in electrical resistance. The RTDs devise are include a sensing element, connection wires which link the measurement instrument to sensing element and finally a support for positioning the element in the process. "The metal sensing element is an electrical resistor that changes resistance with temperature. The element usually contains a coil of wire or 
conductive film with conductors etched or cut into it. It is usually housed in ceramic and sealed with ceramic cement or glass" (Wilson, 2005) (Figure 5).

The correct position of the sensing element is significant. "It must be located where it can reach process temperature quickly. Also, the devices should be adequately secured in high vibration and shock application. the wires between the element and instrument allow resistance measurement from great distances" (Wilson, 2005).

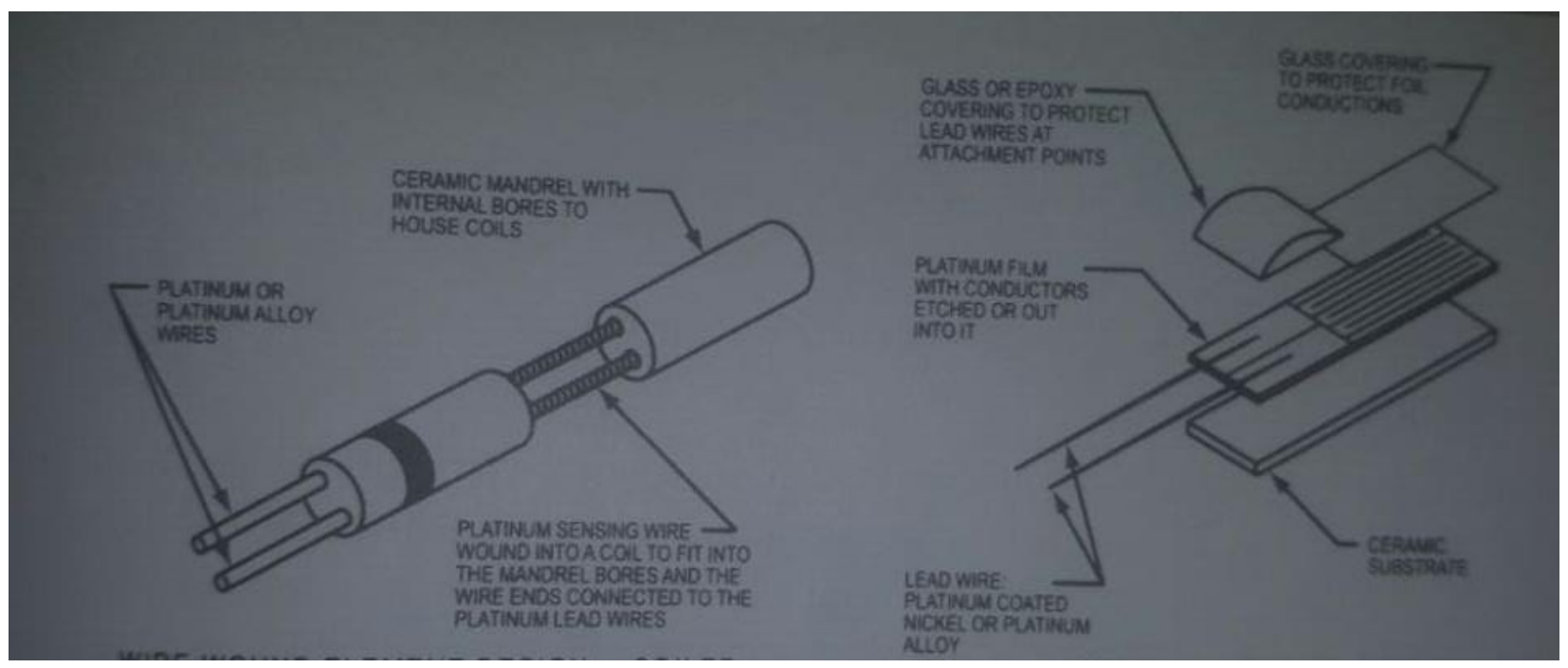

Figure 5 -RTD Sensor (Wilson, 2005)

\subsubsection{Thermistor}

Thermistor or thermally sensitive resistor almost have the same performance as RTDs. In this device, the electrical resistance changes in relation to the temperature. This device is typically consisting of a combination of two or three metal oxides that are sintered in a ceramic base material. Also, there is a lead wires which connects ceramic base to a semiconductor wafer or chip. The chip is covered with epoxy or glass.

There are two type of thermistor exist: positive temperature coefficient (PTC)and negative temperature coefficient (NTC). PTC devise show increase in resistance as temperature rises, while NTC devises show a decrease in resistance or negative slope when temperature 
increase. NTC has a higher degree of sensitivity as the changes in resistance is quite larger. Also, the unique feature of these type of sensor is the small size and rapid response to temperature changes but because of non-linear characteristics of thermistor the signal processing is very complicated.

\subsubsection{Advantages and disadvantages of NTC vs. thermocouple \& RTDs}

NTC thermistor, thermocouple and RTDs are among the most popular thermal sensors. The negative temperature coefficient thermistor (NTC) which is more popular than positive one (PTC) is made of semiconductor material. The feature of this device is as the resistance

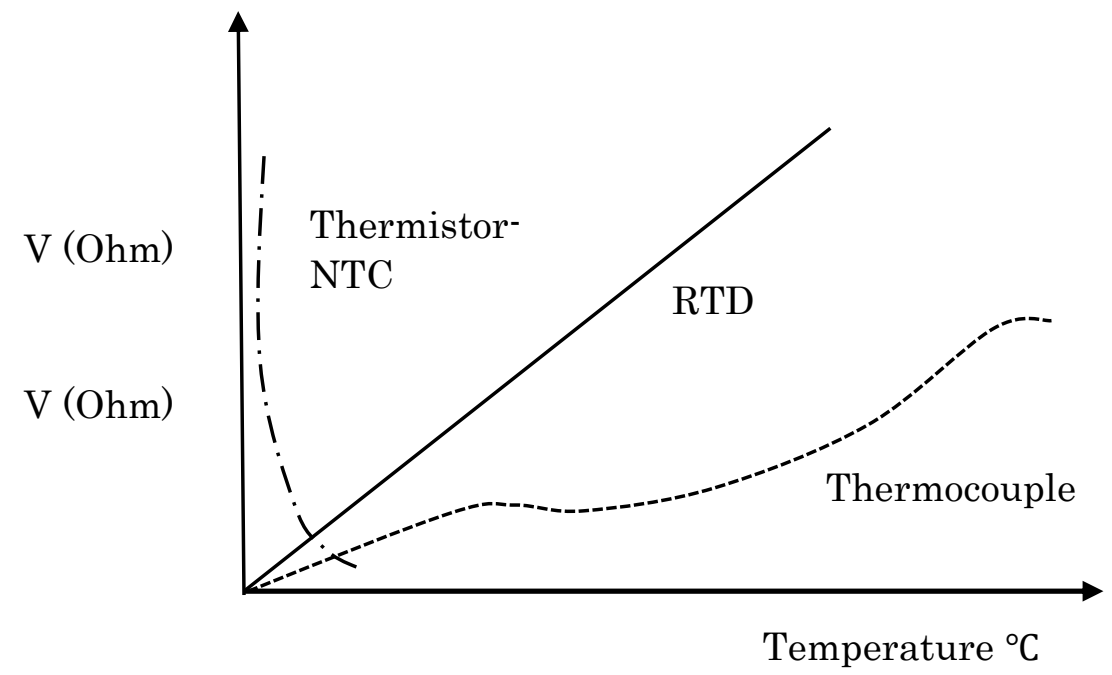

Figure 6--NTC thermistor vs. RTDs and thermocouple

decrease the temperature rises. Thermocouple is most versatile thermometer. It has widest range of temperature compare to other devices but it is not as accurate as thermistor and RTDs. In fact, thermistor is most sensitive and RTD is most accurate thermometer. The main disadvantages of the thermistor are strong non-linearity (Figure 6).

The table 5 illustrates the different characteristics of popular types of sensors, advantages and disadvantages. This classification helps to pick the right type for a specific use. 


\begin{tabular}{|c|c|c|c|c|c|}
\hline \multicolumn{6}{|c|}{ Sensors Advantages and Disadvantages } \\
\hline & Sensitivity & Temp. range & linearity & $\operatorname{cost}$ & Extra feature \\
\hline thermistor & Very high & $\begin{array}{l}\text { Limited } \\
-100^{\circ} \mathrm{C}-300^{\circ} \mathrm{C}\end{array}$ & nonlinear & low & $\begin{array}{l}\text { Fast thermal } \\
\text { response }\end{array}$ \\
\hline Thermocouples & medium & $\begin{array}{l}\text { Very high } \\
-200^{\circ} \mathrm{C}-2315^{\circ} \mathrm{C}\end{array}$ & nonlinear & low & $\begin{array}{l}\text { high Vibration \& } \\
\text { shock resistance }\end{array}$ \\
\hline RTDs & high & $-200^{\circ} \mathrm{C}-650^{\circ} \mathrm{C}$ & Linear & high & High temp. Stability \\
\hline Silicon & medium & $\begin{array}{l}\text { Max. } 200^{\circ} \mathrm{C} \\
\text { (Limited) }\end{array}$ & $\begin{array}{l}\text { Nearly } \\
\text { linear }\end{array}$ & medium & $\begin{array}{l}\text { Ideal for many } \\
\text { application }\end{array}$ \\
\hline
\end{tabular}

Table 5 


\section{Sensor performance:}

The sensor is the most important element on measurement system. If data is distorted or corrupted by the sensor, there is normally difficult or sometimes impossible to fix it. Any sensor, no matter how accurately calibrated, high quality and small it is, it will disturb the measurement site and thus cause some error in temperature measurement. This type of error can apply to any method of sensing (conductive, radiative and convective). Therefore, it is an important duty to minimize error by using correct measurement technic and an appropriate sensor design.

Sensors are often used to make quantifiable measurement, versus qualitative detection. Therefore, analysing the requirement of measurement help to determine the appropriate type of sensor and as the result more accurate data. First step in this process is understanding the quality of measuring object, form and characteristic. Second step is analysing the environment. Often environment impacts the most on measurement system and cause unpredicted errors. Indeed, Sensor and whole measurement system, respond to their environment, not just to measurand. The goal of this project is minimising environmental distraction and maximizing response to desire measurands. That's why appropriate design of sensor is challenging. The environmental factors are not only limited to temperature, pressure and vibration but also factors such as the mounting or attachment of sensor, electromagnetic effects and rate of environmental changes, impact the accuracy of result. A good example is, sensors with high tolerance to extreme temperature but inability to response in rapidly changing it. Third stage is considering the requirements for accuracy (uncertainty) of measurement. Ideal situation is achieving the lowest possible uncertainty. 
Last but not least, the system must be calibrated and traceable based on national standards organization. This part of process is important as without selecting a baseline the uncertainty of any measurement is unknown. Either the whole system must be calibrated or each part of measurement system must be calibrated and overall uncertainty calculated.

\subsection{Accuracy and uncertainty}

Understand the difference between accuracy and uncertainty is Crucial. "Uncertainty is generally defined as the largest expected error between actual and ideal output signals. Sometimes this is quoted as a fraction of the full-scale output or fraction of the reading. For example, a thermometer might be guaranteed accurate to within $5 \%$ full scale output. "Accuracy" is generally considered by metrologists to be a qualitative term, while "uncertainty" is quantitative. For example, one sensor might have better accuracy than another if its uncertainty is $1 \%$ compared to the other with uncertainty of $3 \%$ " Wilson, 2005).

\subsection{Equilibrium and thermal Coupling}

To improve measuring accuracy and certainty it is important to understand factors which impact the most on the system. Different researches done in this field. "One of the main issue is appropriate coupling between the sensor and object" (Fraden, 2010). The two basic

methods of signal processing as the temperature sensors expose to heat are equilibrium and predictive. "In the equilibrium method, a temperature measurement is complete when there is not significant thermal gradient exists between the measured surface and the sensing element inside the probe. In other words, a thermal equilibrium is reached between the sensor and object of measurement when energy exchange becomes negligible" (Fraden, 2010). While in the predictive method system works differently. As the system does not 
have enough time to reach the equilibrium therefore it predicts the number via a computation through the rate of the sensor's temperature change. In both process the sensor dimension (size) impacts on the response time. The smaller the sensor the faster the response time. Many factors impact on coupling between object and sensor.

1. One of the main reason is inappropriate junction design. Any non-uniform contact between junction and surface cause error in the whole system especially nonuniform lap joints and butt joints.

2. Another factor is use of an electrical insulating layer between junction and apparatus. "The insulating layer, although electrically insulating, contains a substantial thermal mass and thereby provides a thermal loading effect on the thermocouple junction, thereby resulting in thermal measurement error and a slow response time" (United State of America Patent No. 6257758, 2001).

3. The other factor that cause equilibrium issue is related to material selection. Different metals have different resistivity. The most popular metal used in thermal sensor are Platinum, nickel and copper. Platinum is one of the most popular metal which has temperature range of $-260^{\circ} \mathrm{C}$ to $750^{\circ} \mathrm{C}$, good linearity and stability. Also, copper is widely use in this industry. It is almost linear, but it has a lower resistance and it oxidize faster. Table 6 demonstrate the temperature range of these three metals. Following Image illustrates the relation of temperature versus resistance (Figure 7).

\begin{tabular}{|c|c|c|}
\hline Material & Span C & R100/R0 \\
\hline Platinum & -260 to 270 & 1.385 \\
\hline Nickel & -80 to 30 & 1.672 \\
\hline Copper & -200 to 260 & 1.427 \\
\hline
\end{tabular}

Table 6- Comparison of popular sensor metals 


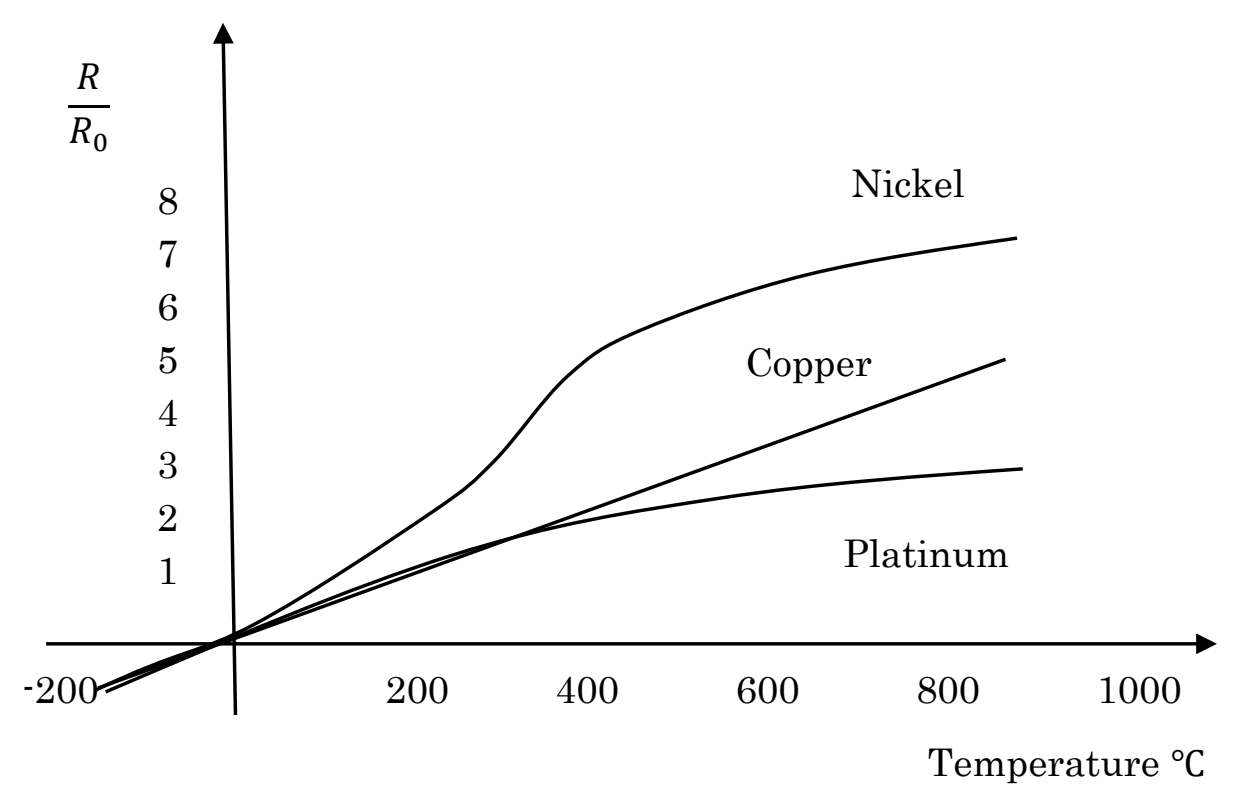

Figure 7- Temperature vs. resistance.

$\mathrm{R} 100$ is the resistance at $100^{\circ} \mathrm{C}$ and $\mathrm{RO}$ is resistance at $0^{\circ} \mathrm{C}$

4. Size of the sensor is another factor that impacts on equilibrium. Different studies shown that the smaller the sensor the faster response time and better decoupling from environment. Jacob Farden in "Handbook of modern sensors" was proved this fact with the help of mathematical model.

5. The other challenging factor that reduce representativeness of sensor measurement is presence of third party in contact with the sensor. Indeed, "the sensor is coupled not only to the object which temperature is measured, but also to other items" (Fraden, 2010). Some material such as adhesives impact the accuracy of result as they create heat transfer resistance between the object and the sensor. In many cases, even the surrounding air can cause this type of errors on the system. In fact, never at any moment of time the temperature between object $T_{B}$ and sensor $T_{S}$ are 100 percent equal. The goal of the equilibrium measurement is to bring $\mathrm{T}_{\mathrm{S}}$ as close to $\mathrm{T}_{\mathrm{B}}$ as possible. "In a typical temperature sensor one end of the cable is connected to the sensor while the other end is subjected to another temperature, for example, the 
ambient temperature $\mathrm{T}_{0}$ that may be quite different from that of the object. The cable conducts both an electric signal and some heat from or to the sensor" (Fraden, 2010). Figure 8 illustrates a thermal circuit which includes the object, sensor, environment, and thermal resistances $\mathrm{r} 1$ and $\mathrm{r} 2$.

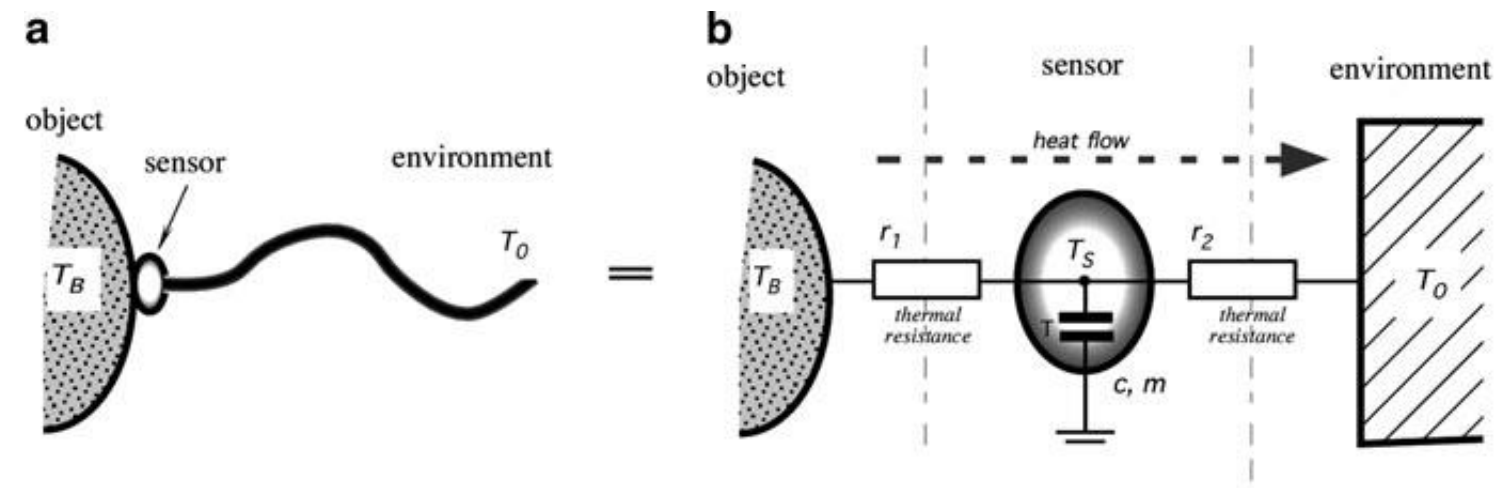

Figure 8- Resistance between sensor, environment and object (Fraden, 2010)

The existence of resistance ( $\mathrm{r} 1$ and $\mathrm{r} 2$ ) clearly shows that the temperature of sensor and object never can be equal but there are methods that help to increase accuracy and reduce uncertainty. Based on research done by Edward B. Coy With increasing the insertion depth the resistance between object and sensor is decrease. In his research, he was recommended to embed the sensor into a wall as the external surface is expose to environment and corrosion. "do not rely on surface temperature measurements but embed the sensors within the wall where they are protected from erosion" (Coy, 2010).

This means that for minimizing the measurement error, must improve thermal coupling between the object and the sensor and decouple the sensor from the surroundings as much as practical (Figure 9). Image below illustrate a sensor embedded into an object. as the sensor and part of cable is inside the hole, it was 
suggested to fill the empty space with insulation material to prevent heat lost. This way the measurement result become significantly more accurate.

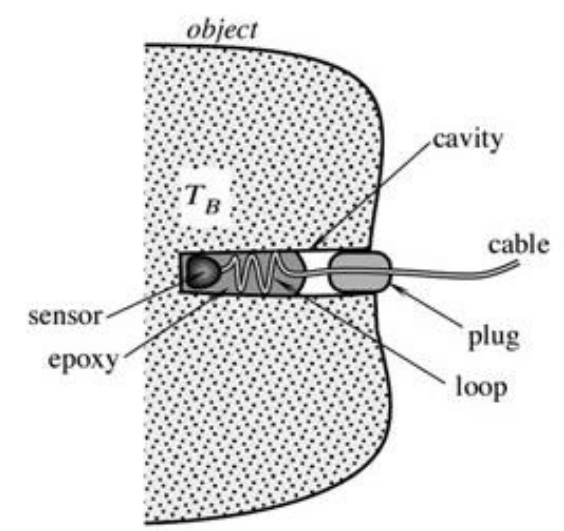

Figure 9- Embedding sensor into an object

To protect the cavity from heat lost preferably fill the whole with thermal grease, epoxy or other method (Fraden, 2010)

As mentioned above several factors directly impact on the accuracy of sensor's output such as, appropriate sensor design specially at the junctions, material selection, size and shape of the sensor and coupling. In this research project the impact of coupling on measurement representativeness will be investigated. 


\section{Simulation}

The proper coupling between sensor and surface is important as any deficiency can cause errors in measurement. The more the sensor decouple from surrounding environment, the more accurate data generates. This separation can be created by covering sensor into a package, glass shield or embedding it into the object. Embedding the sensor into the object is one of the most accurate method as part of the sensor or the whole will be in contact with the object. The drawback of this system is that it is not always practical. The main goal of this research project is evaluating and comparing sensor accuracy in different levels of tightness. How does the result change with embedding the sensor into the object? Are these differences noticeable? And does external weather condition impact on sensor accuracy? For this purpose, by the help of Matlab, an engineering software, and Simulink, a graphical programming environment for modeling, simulating and analyzing multidomain dynamic systems, a model (3-bedroom house) developed. The focus of this project is on the bedroom \#1 (B1).

Figure 10 illustrate the details of bedroom 1 . The red dotted line shows impact of exterior temperature and solar radiation on different layers of wall assembly, from exterior to interior. Also, the green dotted line shows temperature of interior partition wall, ceiling and floor. The internal wall surface temperature of subjected wall depends on different indoor surfaces temperature, room occupancy, Toronto climate data and type of the wall assembly. 


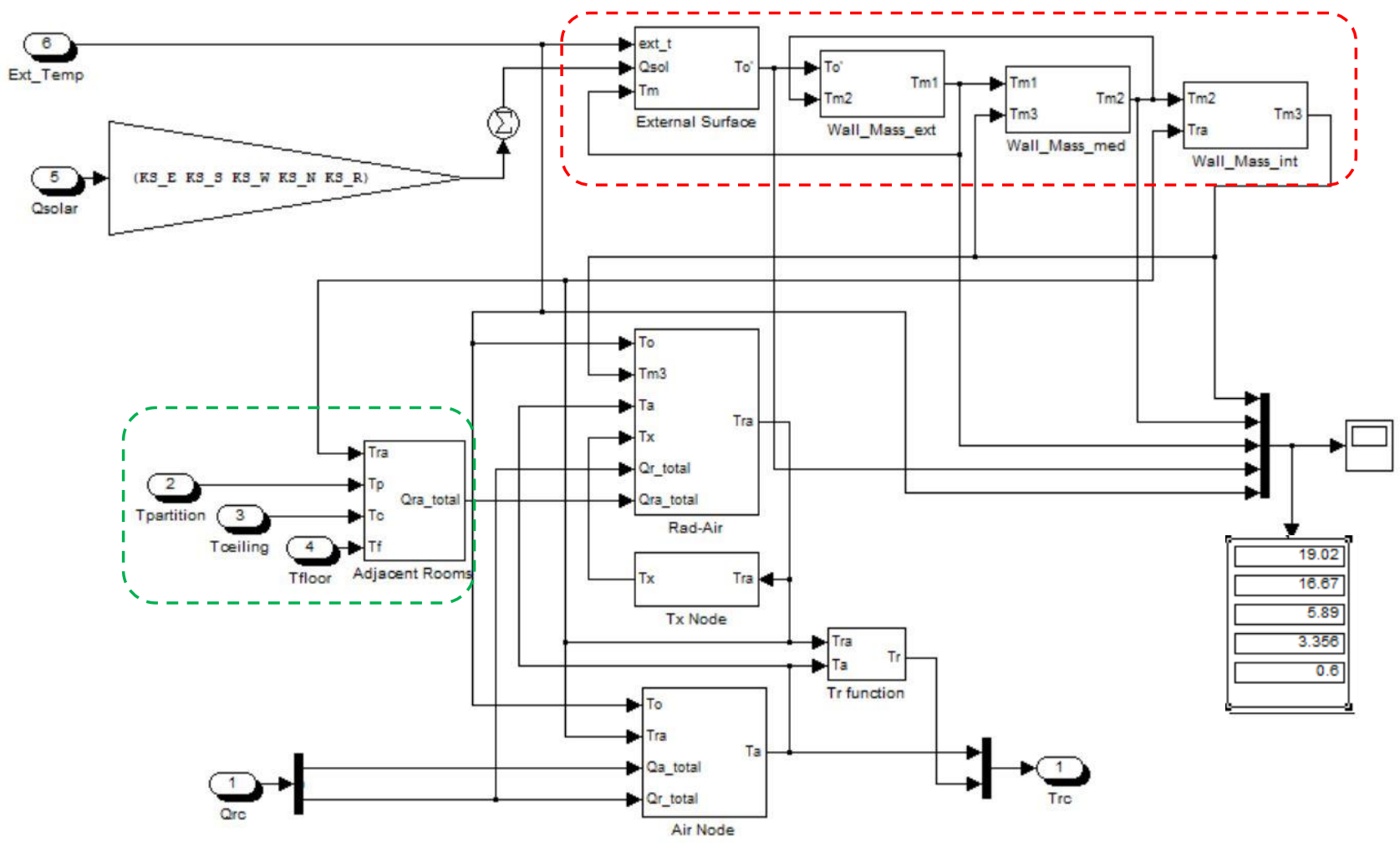

Figure 10- Simulink construction detail

The focus of this project is on sensor performance. In this project the room temperature (ambient air) and wall surface temperature designed as input and the sensor generates the data as the output. In this project, five different level of tightness studied (very loose coupling between sensor and interior wall surface to very tight (embedded sensor in to the wall) (Figure 11). The simulation is happening in three different days. From very cold, January $1^{\text {st }}$, cold, February $15^{\text {th }}$, and average, April $1^{\text {st }}$. the length of simulation is 24 hours (1440 minute). 


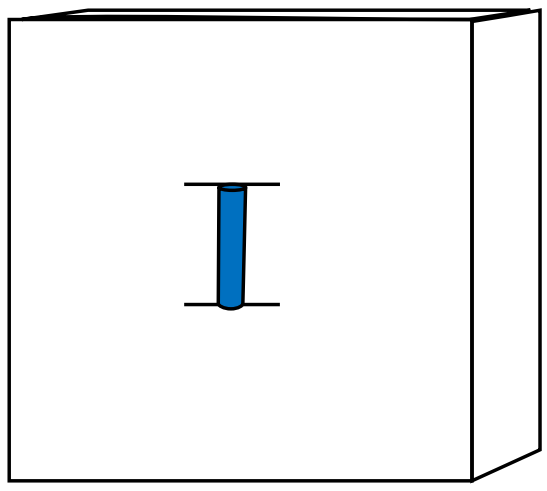

Coupling between wall surface and sensor

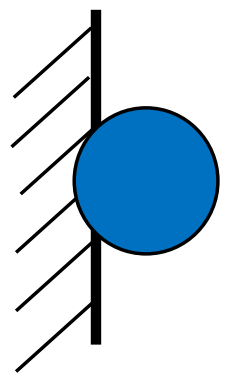

Shape $1-10 \%$

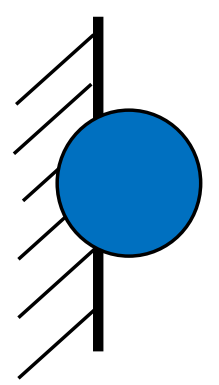

Shape $2-30 \%$

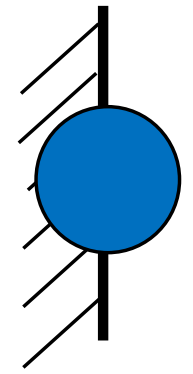

Shape $3-50 \%$

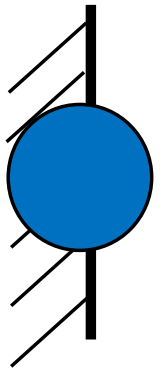

Shape $4-70 \%$

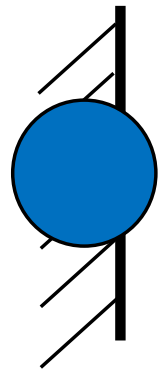

Shape $5-90 \%$

Figure 11-Level of Tightness

Before analyzing these five scenarios by Simulink, this experiment can be proven by mathematical model (Figure 12) In this model, A1 assumed to be the area between air and sensor and A2, area between sensor and wall. $\mathrm{R} 1$ and $\mathrm{R} 2$ respectively are showing thermal resistance air and wall ( $\mathrm{R}$ value). $\mathrm{R} 2$ can be varied based on different wall assemblies. The goal of this mathematical model is to prove that the larger contacted area between wall and sensor creates smaller resistance and as the result more accurate data. 


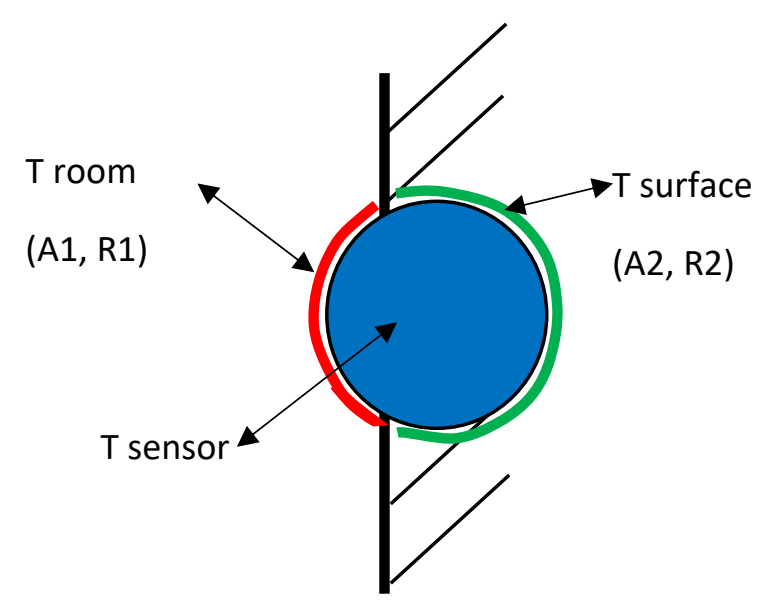

Figure $12-70 \%$ insertion depth

Heat transfer rate between sensor and air:

$$
Q_{1}=\frac{A_{1}}{R_{1}}\left(T_{\text {room }}-T_{\text {Sensor }}\right)
$$

Heat transfer from sensor to surface

$$
Q_{1}=\frac{A_{2}}{R_{2}}\left(T_{\text {Surface }}-T_{\text {Sensor }}\right)
$$

Heat energy balance of sensor

$$
C * \frac{d T_{\text {Sensor }}}{d t}=Q_{1}+Q_{2}
$$

Specific heat capacity formula is $C=\rho c v$ and $\mathrm{v}$, volume of cylinder is equal to $v=\pi r^{2} h$ therefore, the formula will be $C=(\rho c)_{\text {Copper }}\left(\pi r^{2} h\right)_{\text {cylender }}$. The result of this equation is a number which will be called "X". In the [5] and [4], $A_{1}$ and $A_{2}$ are calculating as follows 


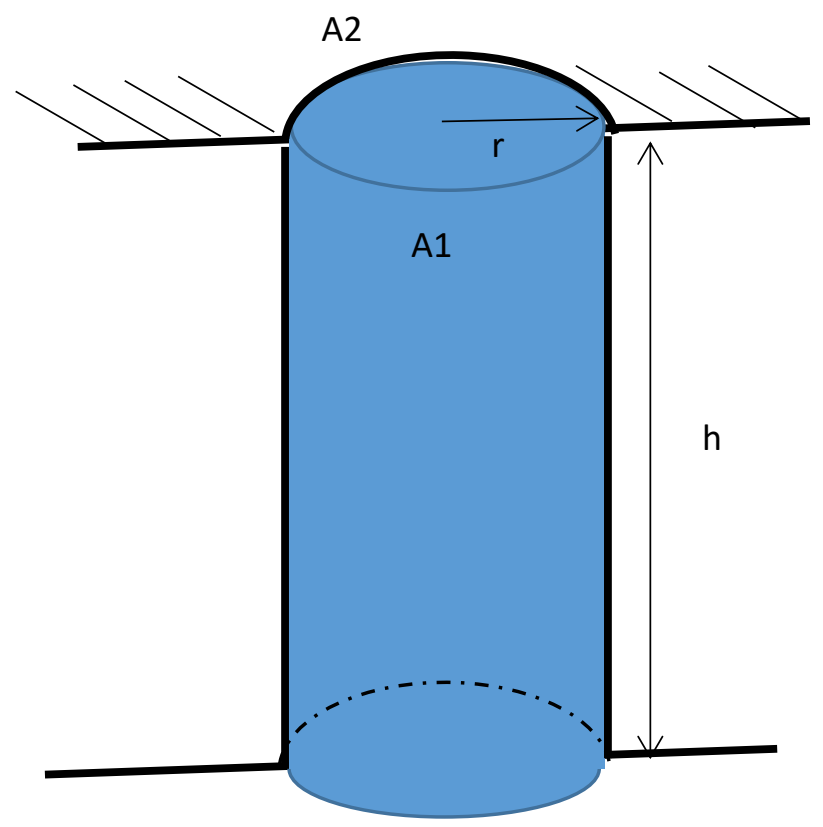

Figure 13- Cylindrical sensor, Insertion detail

$$
\begin{gathered}
A 1=\frac{1.5}{5}\left[2 \pi r^{2}+2 \pi r h\right] \\
A 2=\frac{3.5}{5}\left[2 \pi r^{2}+2 \pi r h\right] \\
A 1<A 2
\end{gathered}
$$

In this case $\mathrm{R} 1$ is air resistant $\left(\frac{m^{2} *^{\circ} \mathrm{C}}{W}\right)$. And $\mathrm{R} 2$ is object resistance which depends on wall material. In this formula if A1 (external surface) decrease toward zero (it means no contact between air and sensor) therefore, A2 will be 100 percent (embedding sensor into object). In this case, we can assume first part of formula will be equal to zero [7].

$$
\begin{gathered}
\mathrm{X}=\frac{\mathrm{A} 1}{\mathrm{R} 1}\left(\mathrm{~T}_{\text {room }}-\mathrm{T}_{\text {surface }}\right)+\frac{\mathrm{A} 2}{\mathrm{R} 2}\left(\mathrm{~T}_{\text {surface }}-\mathrm{T}_{\text {sensor }}\right) \\
\mathrm{X}=0+\frac{\mathrm{A} 2}{\mathrm{R} 2}\left(\mathrm{~T}_{\text {surface }}-\mathrm{T}_{\text {sensor }}\right) \\
\mathrm{X} * \frac{R 2}{\mathrm{~A} 2}=\left(\mathrm{T}_{\text {surface }}-\mathrm{T}_{\text {sensor }}\right)
\end{gathered}
$$


In this formula $\mathrm{A} 2 \approx 1$, and $\mathrm{X}$ is a number. Therefore, the temperature difference between surface and sensors has direct relationship with the amount of R2. As the sensor is embedded into object, the temperature of sensor and object tend to be very close although this number never can be equal. This temperature difference is called temperature error. The smaller temperature error proves better decoupling between sensor and environment therefore, smaller thermal resistance ( $\mathrm{R}$ value) between surface and sensor. As the resistance become smaller the data generated by sensor will be more accurate. Another way of measuring this error is by using "Root mean square error" (RMSE).

In fact, in this procedure other factors such as length of contact time, size and shape of sensor and sensor material are significant and impact on data accuracy. For example, selecting the appropriate sensor material or optimal shape of sensor can decrease resistance and increase heat transfer from object to the sensor. With proper design, depth of insertion and material data representativeness can be optimized. The following graphs is showing relationship between resistance and level of tightness (Figure 14).

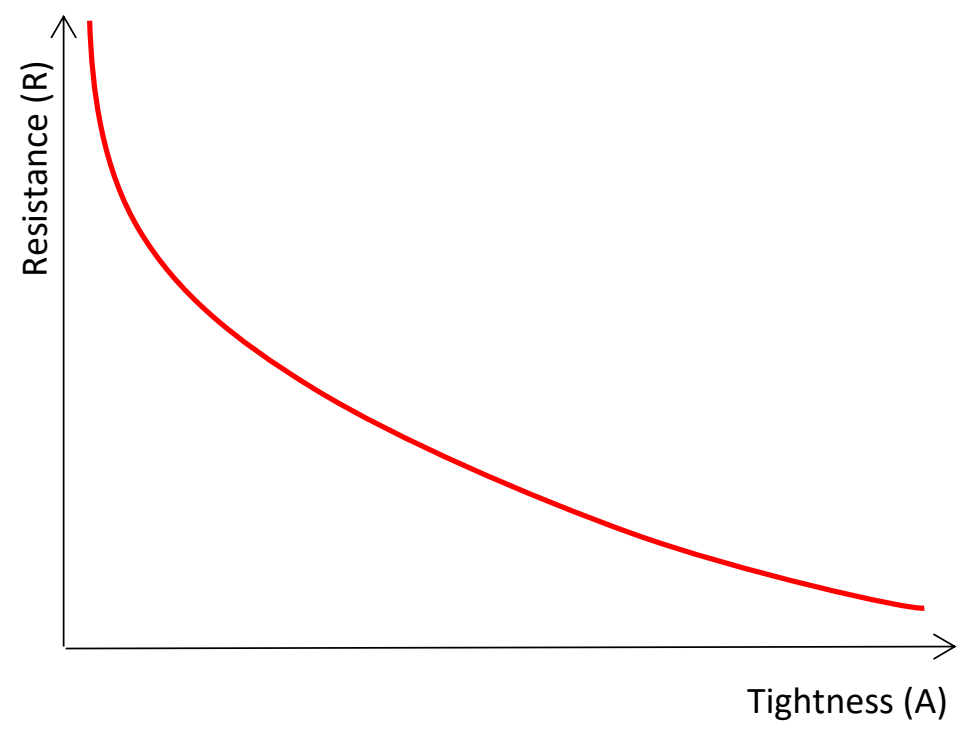

Figure 14- Thermal resistance vs. level of tightness 
The rest of this research project focus on five different levels of tightness between sensor and surface in three days with different weather condition. Each simulation includes 5 different graphs which each graph defined by three colors. Green is indication of indoor air temperature, red is expected wall surface temperature and blue is predicted temperature by sensor.

\subsection{January $1^{\text {st }}$}

This set of simulation done in January $1^{\text {st }}$ for duration of 24 hours. Based on Canada climate data January is coldest months in Ontario. The contact level between sensor and wall in figures 15 and 16 are $10 \%$ and $30 \%$. As graphs below are showing, the sensor temperature is highly under the influence of surrounding environment (room temperature). These graphs illustrate that the surface temperature during non-occupancy hours drops to $19^{\circ} \mathrm{C}$ and at occupancy hours it reaches to almost $22^{\circ} \mathrm{C}$.

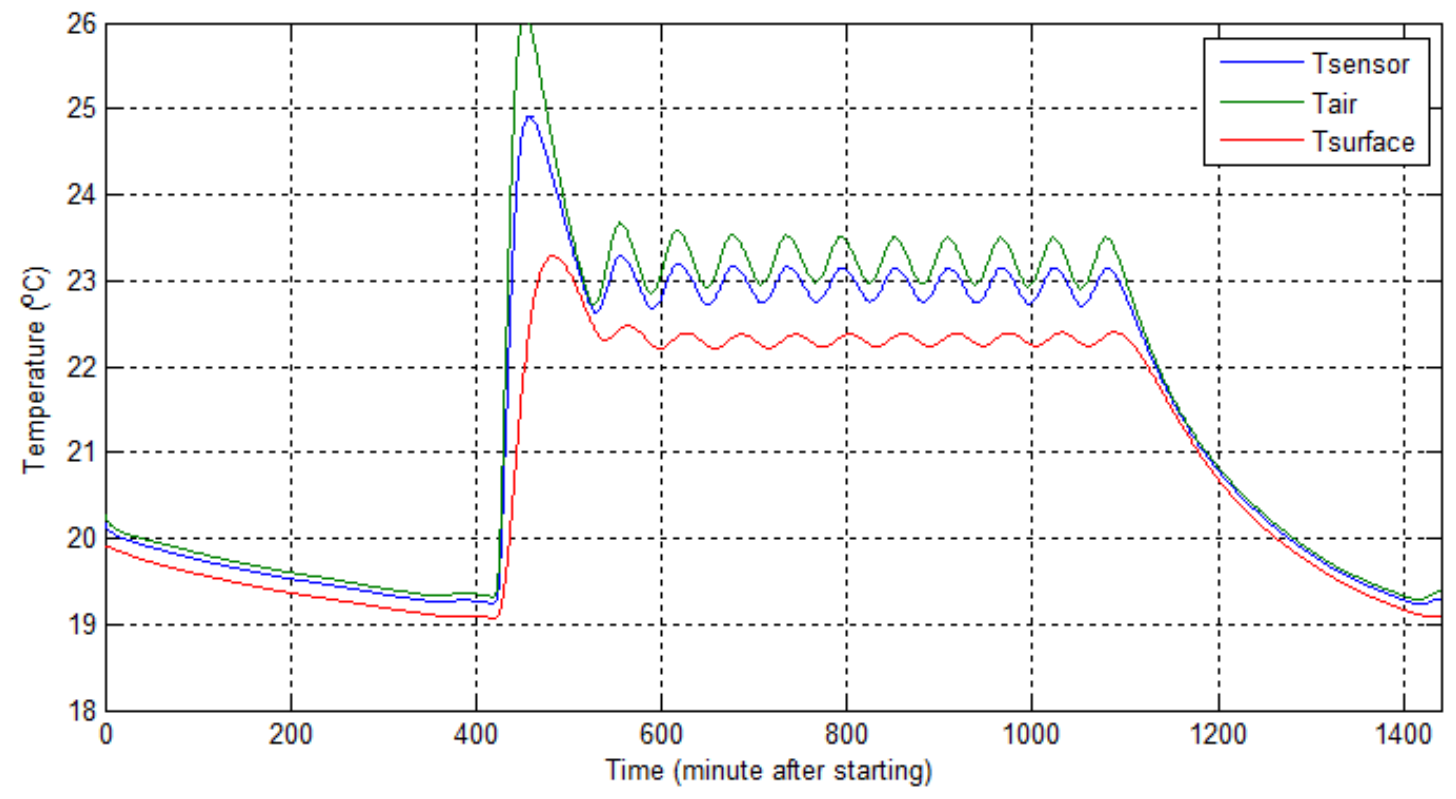

Figure 15--Very loose, January 1st 


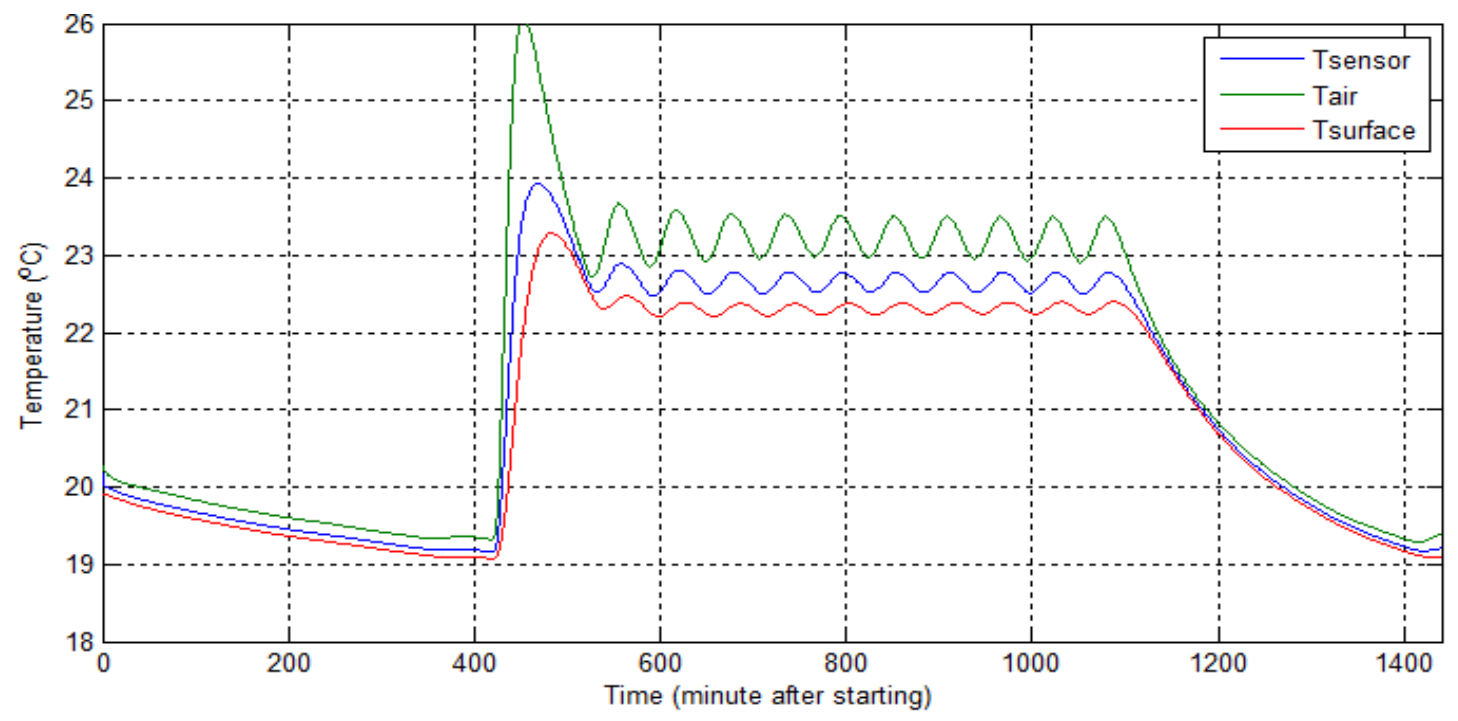

Figure 16-- Loose, January 1st

Figure 17, 18 and 19 are showing 50, 70 and 90 percent contact level. In all three situations, it is noticeable that the sensor performance improves. Figure 19 illustrates a very good decoupling of sensor from environment. Based on Simulink data, with increasing the level of tightness the temperature difference (FMSE) between the sensor and surface decrease while the temperature difference between the sensor and indoor air temperature increase. Table 7 and graph (Figure 20) are illustrating these results.

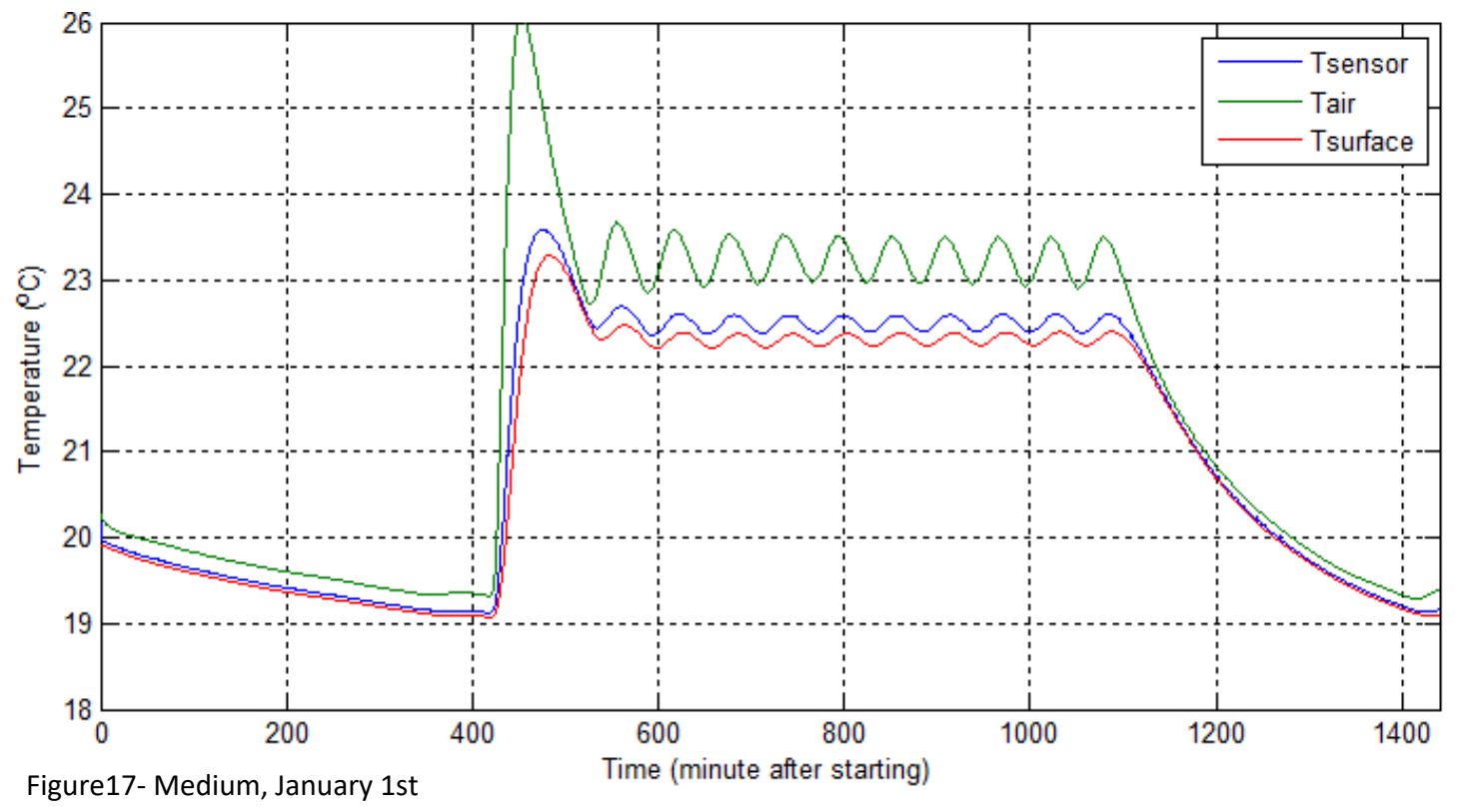




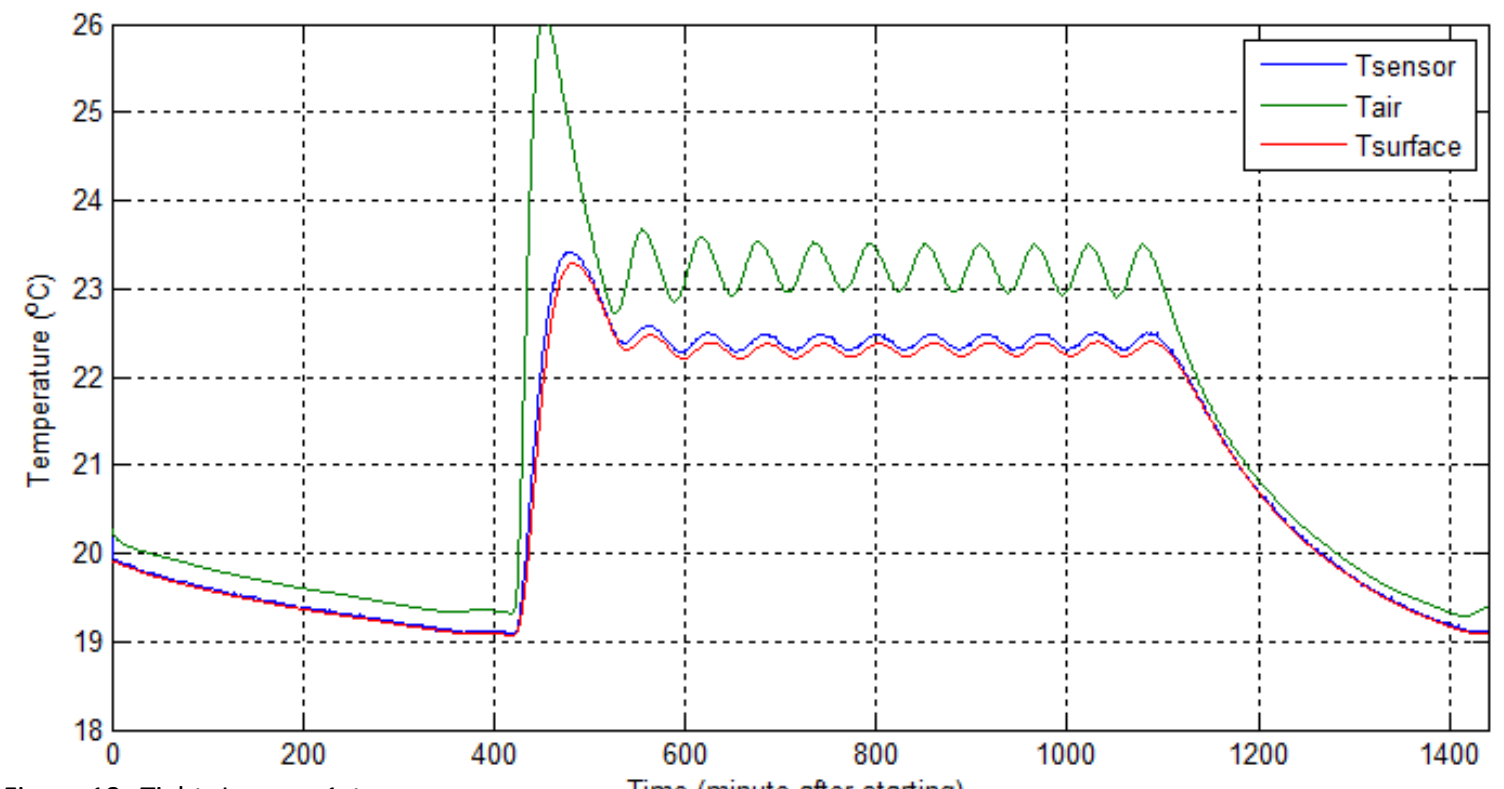

Figure 18--Tight, January 1st

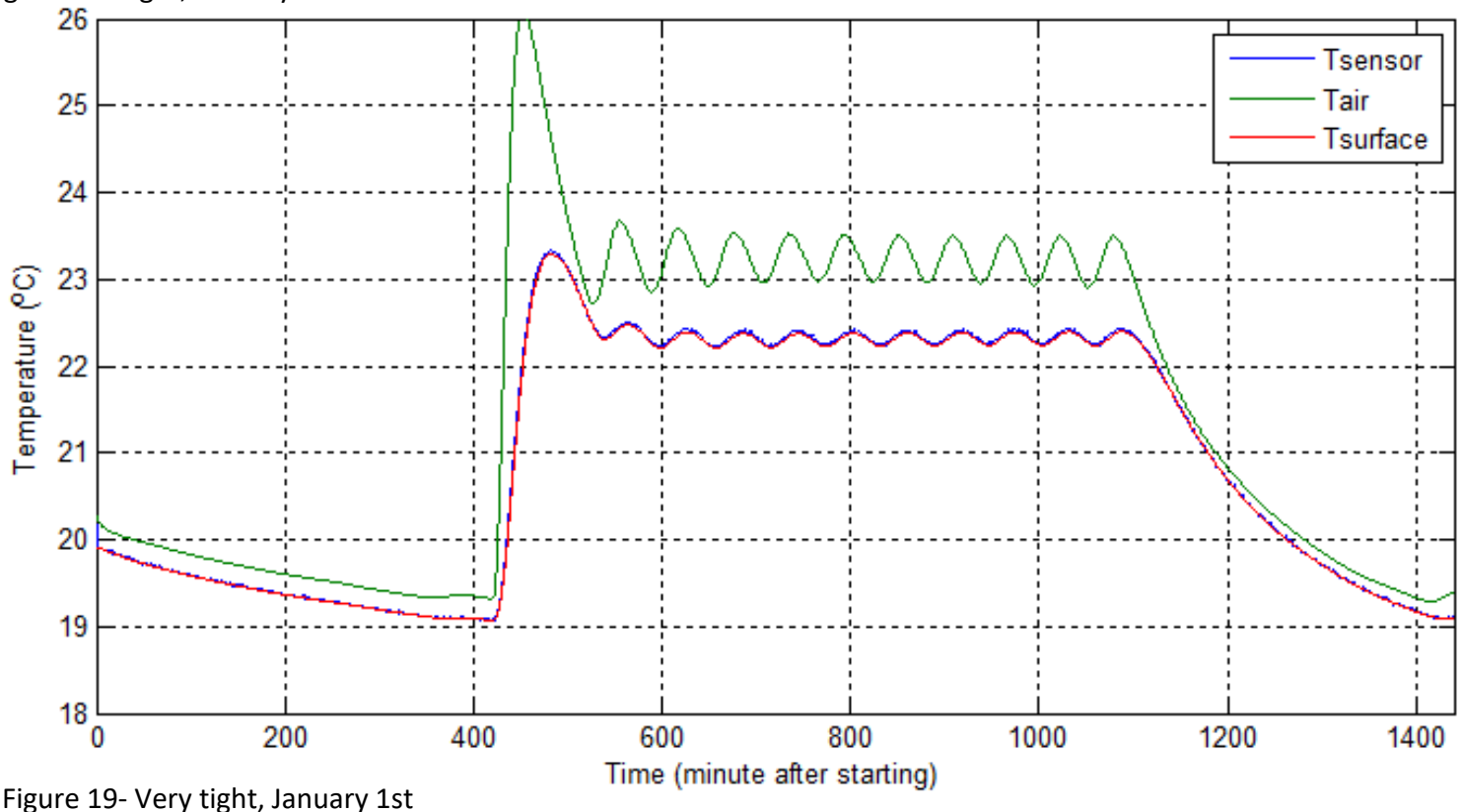

Figure 19- Very tight, January 1st

Table 7

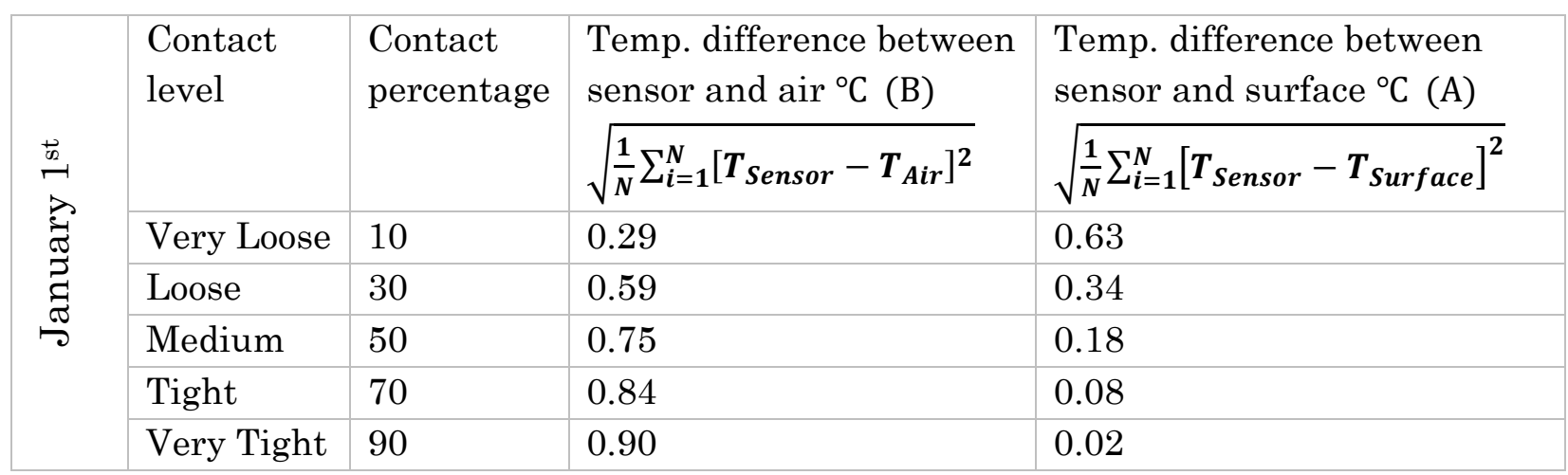


Figure 20 illustrates the opposite performance of column A and B in table 7. As the tightness between sensor and surface increase, the temperature difference between sensor and surface decrease. The gray line is indicating this movement. While the amount of temperature difference between air and sensor which is shown by blue line increase as a result impact of ambient air on sensor decrease.

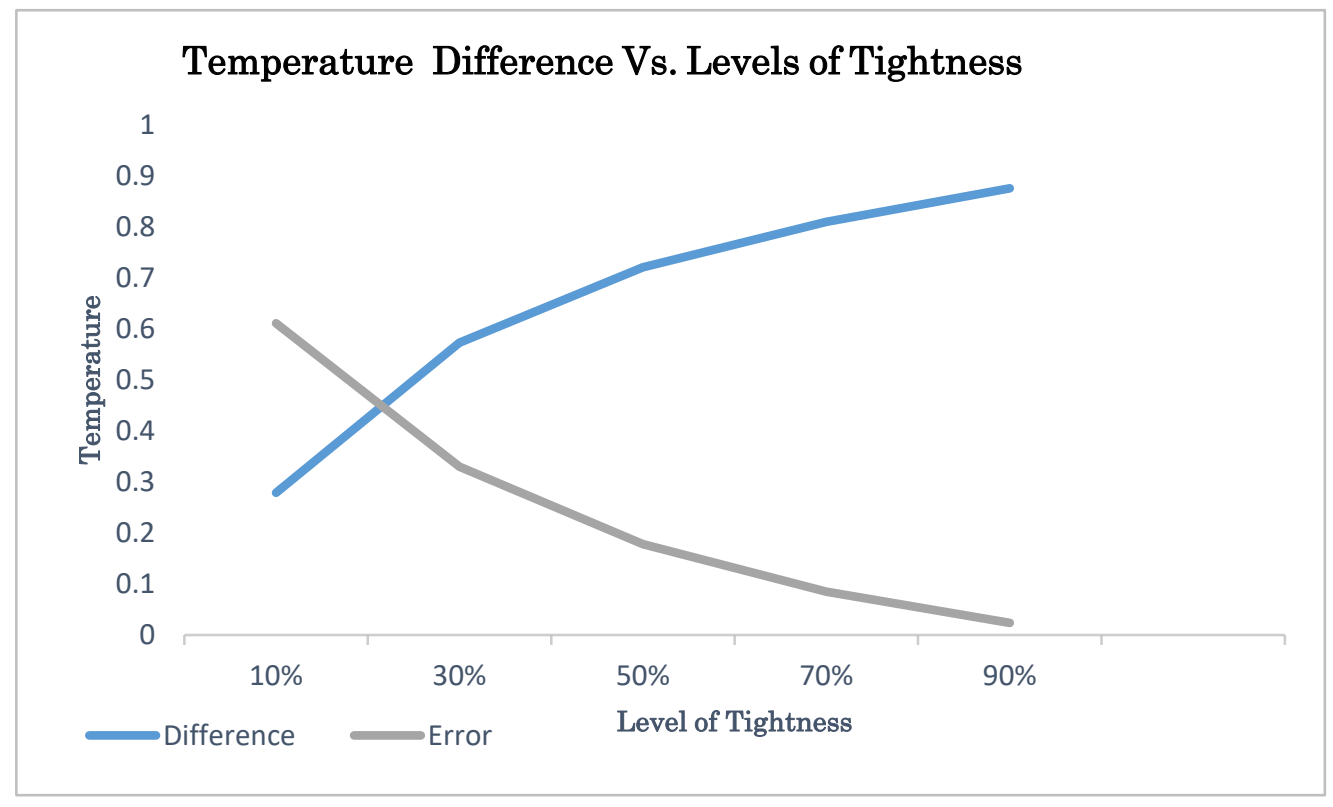

Figure 20-Temperature difference (column A\&B) Vs. levels of tightness

Gray: Temperature difference (sensor \& object). Blue: temperature difference (sensor \& environment) 


\subsection{February $15^{\text {th }}$}

The second set of simulation done in February $15^{\text {th }}$ with 45-day difference from the first simulation. Based on Canadian climate data February is second coldest month in the year, with average temperature of $-4.1^{\circ} \mathrm{C}$ (Norwegian meteorological institute, 2016).

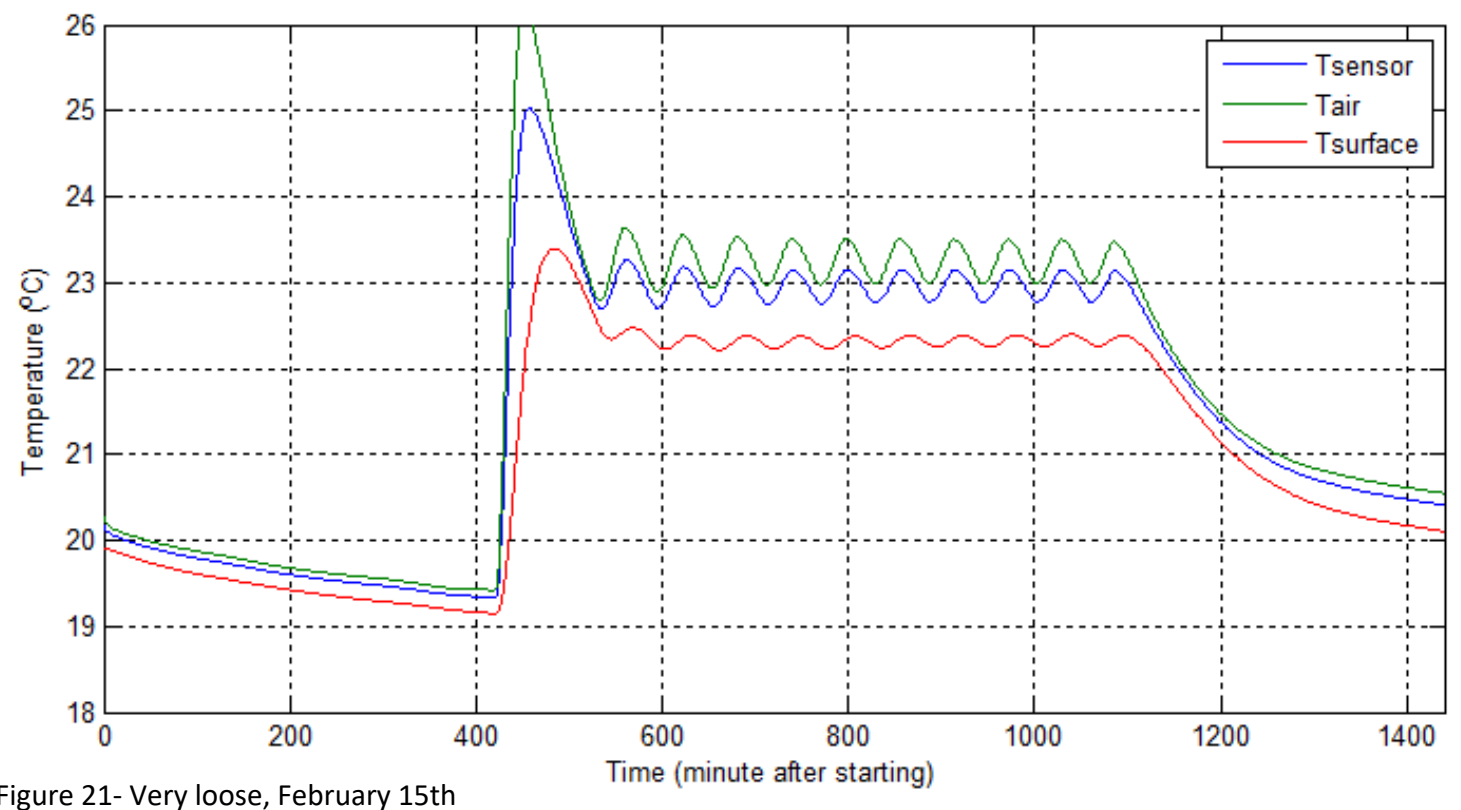

Figure 21- Very loose, February 15th

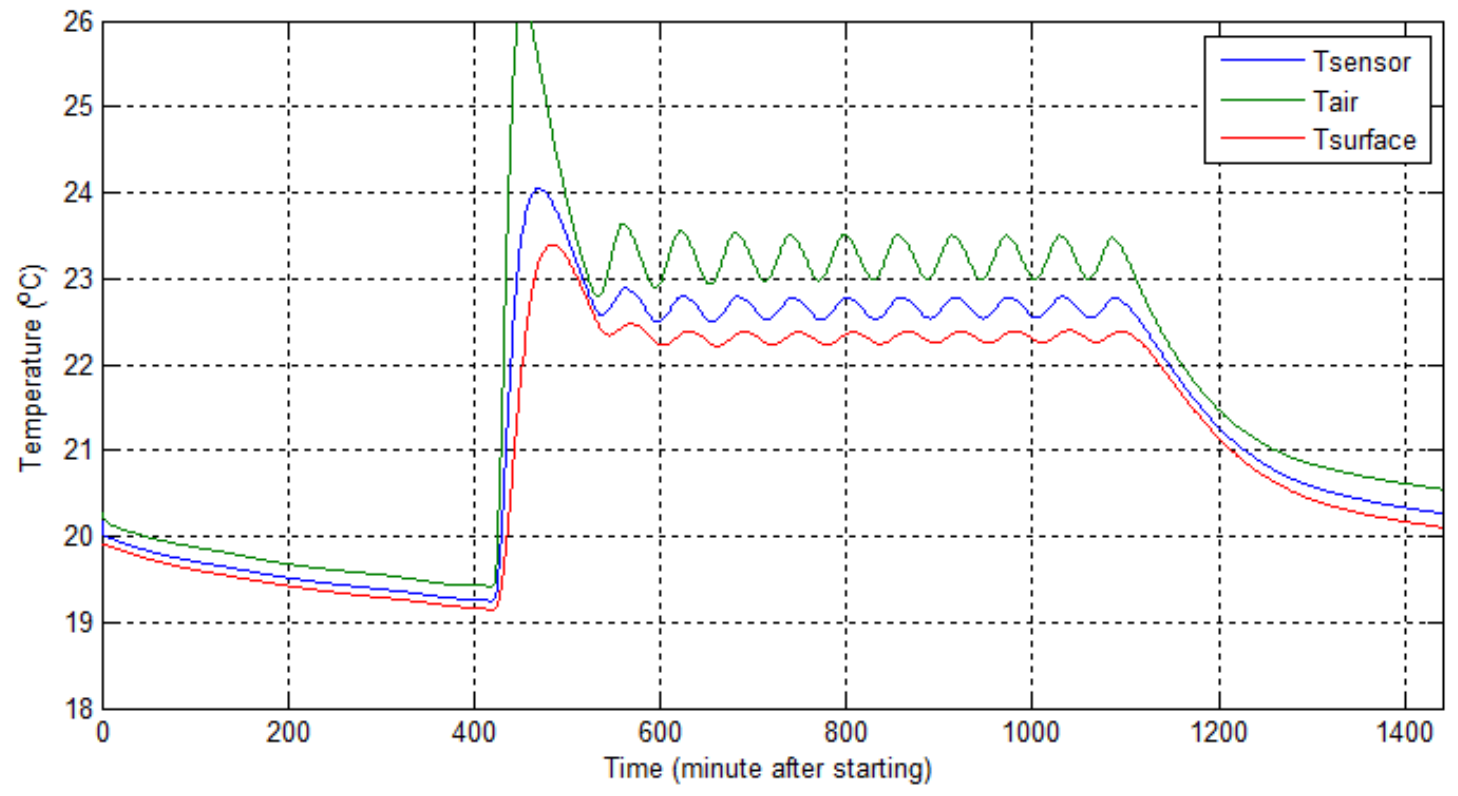

Figure 22- Loose, February 15th 
The graphs from January $1^{\text {st }}$ and February $15^{\text {th }}$ are very similar also the sensor's results are very close. In both sets of simulation with increasing the levels of tightness the blue line, sensor, moved toward red, wall surface.

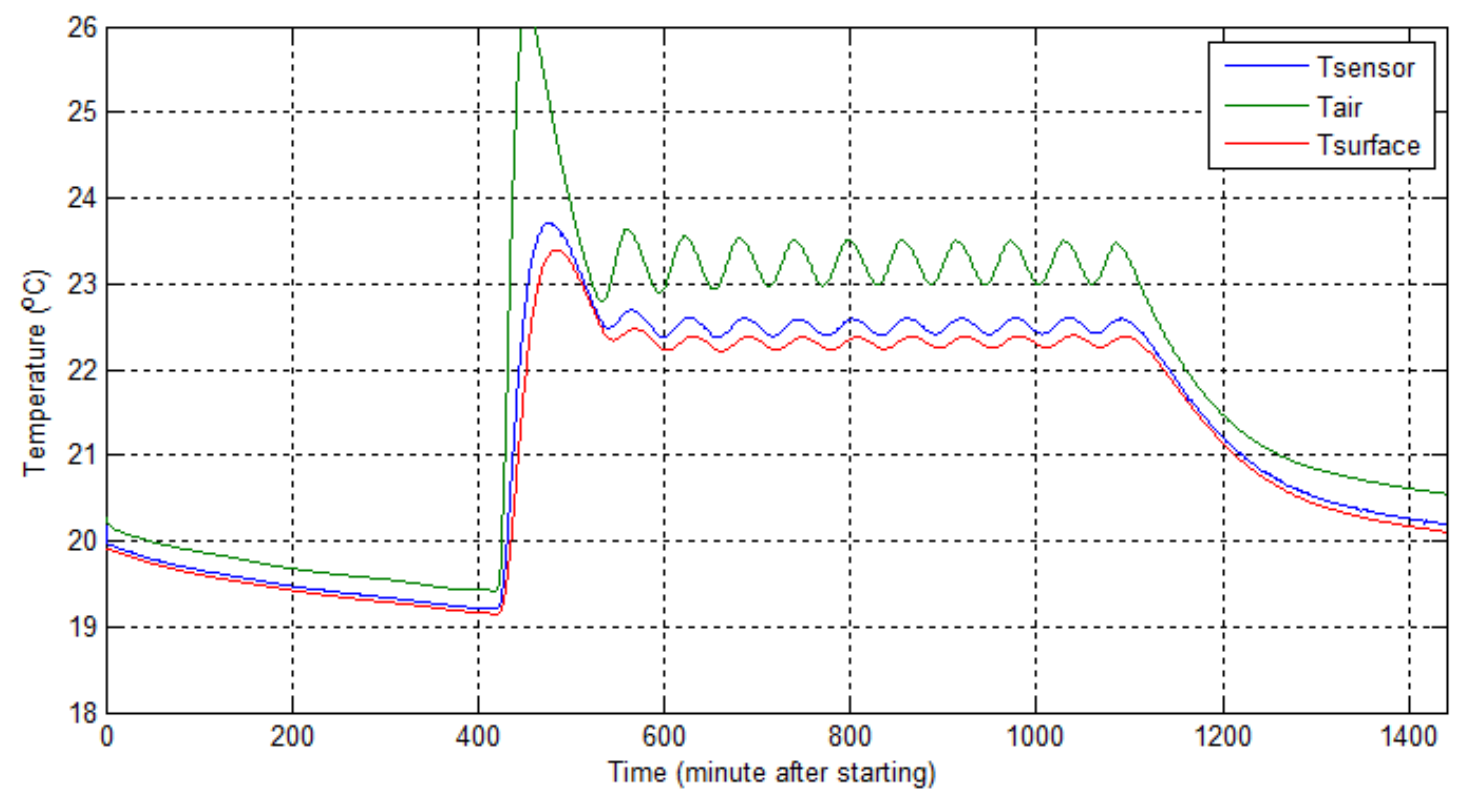

Figure 23-Medium, February 15th

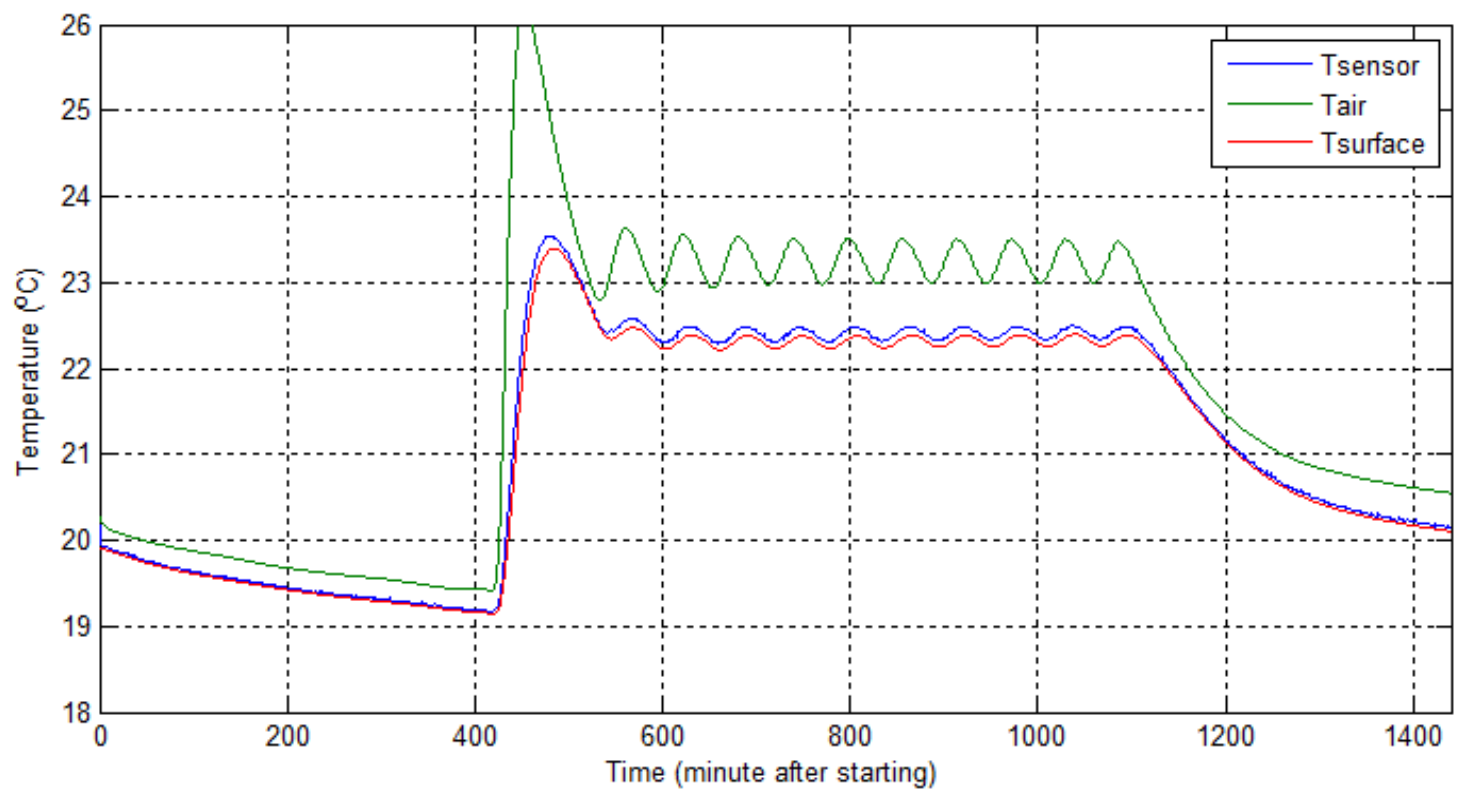

Figure 24- Tight, February 15th 


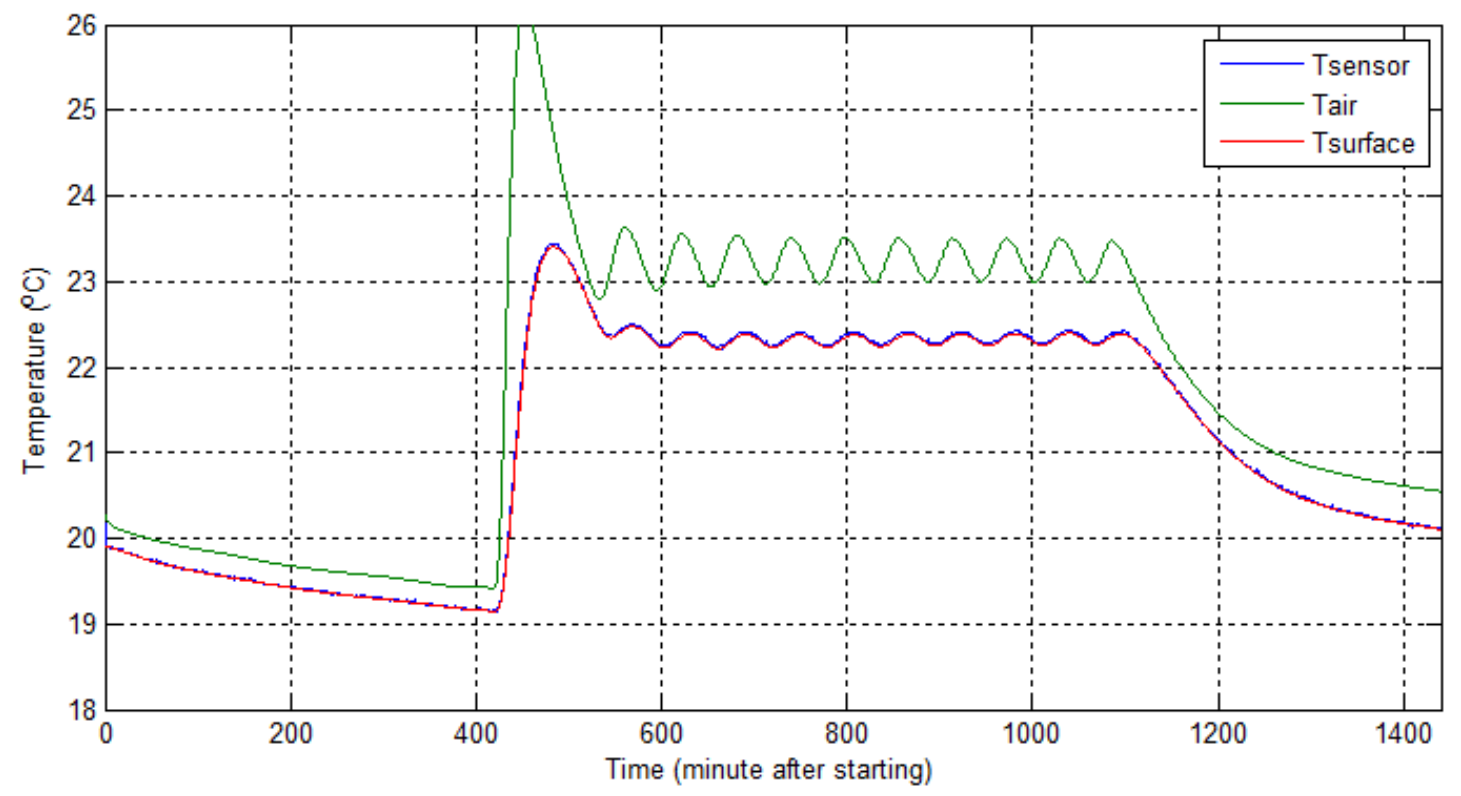

Figure 25- Very tight, February 15th

All simulation in the January $1^{\text {st }}$ and February $15^{\text {th }}$ are showing a big temperature difference between non-occupancy and occupancy hours. This temperature fluctuation is pointing out at system's energy saving mode. It means that in setback hours heating radiator works at minimum amount.

Table 8 is showing the root mean square errors (RMSE) for sensor and air as well as sensor and surface. As it is noticeable with increasing insertion depth, the temperature difference between surface and sensor decrease. This is indication of good performance of system and improvement at measurement representativeness.

\begin{tabular}{|c|c|c|c|c|}
\hline \multirow{7}{*}{ 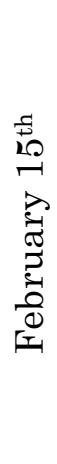 } & $\begin{array}{l}\text { Contact } \\
\text { level }\end{array}$ & $\begin{array}{l}\text { Contact } \\
\text { percentage }\end{array}$ & $\begin{array}{l}\text { Temp. difference between } \\
\text { sensor and air }{ }^{\circ} \mathrm{C}\end{array}$ & $\begin{array}{l}\text { Temp. difference between } \\
\text { sensor and surface }{ }^{\circ} \mathrm{C}\end{array}$ \\
\hline & & & $\sqrt{\frac{1}{N}} \sum_{i=1}^{N}\left[T_{\text {Sensor }}-T_{A i r}\right]^{2}$ & $\sqrt{\frac{1}{N} \sum_{i=1}^{N}\left[T_{\text {Sensor }}-T_{\text {Surface }}\right]^{2}}$ \\
\hline & Very Loose & 10 & 0.27 & 0.61 \\
\hline & Loose & 30 & 0.57 & 0.32 \\
\hline & Medium & 50 & 0.72 & 0.17 \\
\hline & Tight & 70 & 0.80 & 0.08 \\
\hline & Very Tight & 90 & 0.87 & 0.02 \\
\hline
\end{tabular}

Table 8 
The Figure 26 is comparing the temperature difference between sensor and surface in two dates of January $1^{\text {st }}$ and February $15^{\text {th }}$. As the graph is showing at 10 percent tightness the temperature difference between two dates are more visible while with moving toward 100 percent tightness the difference between error getting smaller. This means that after certain point of tightness, it is very difficult (impossible) to reach $0^{\circ} \mathrm{C}$ error.

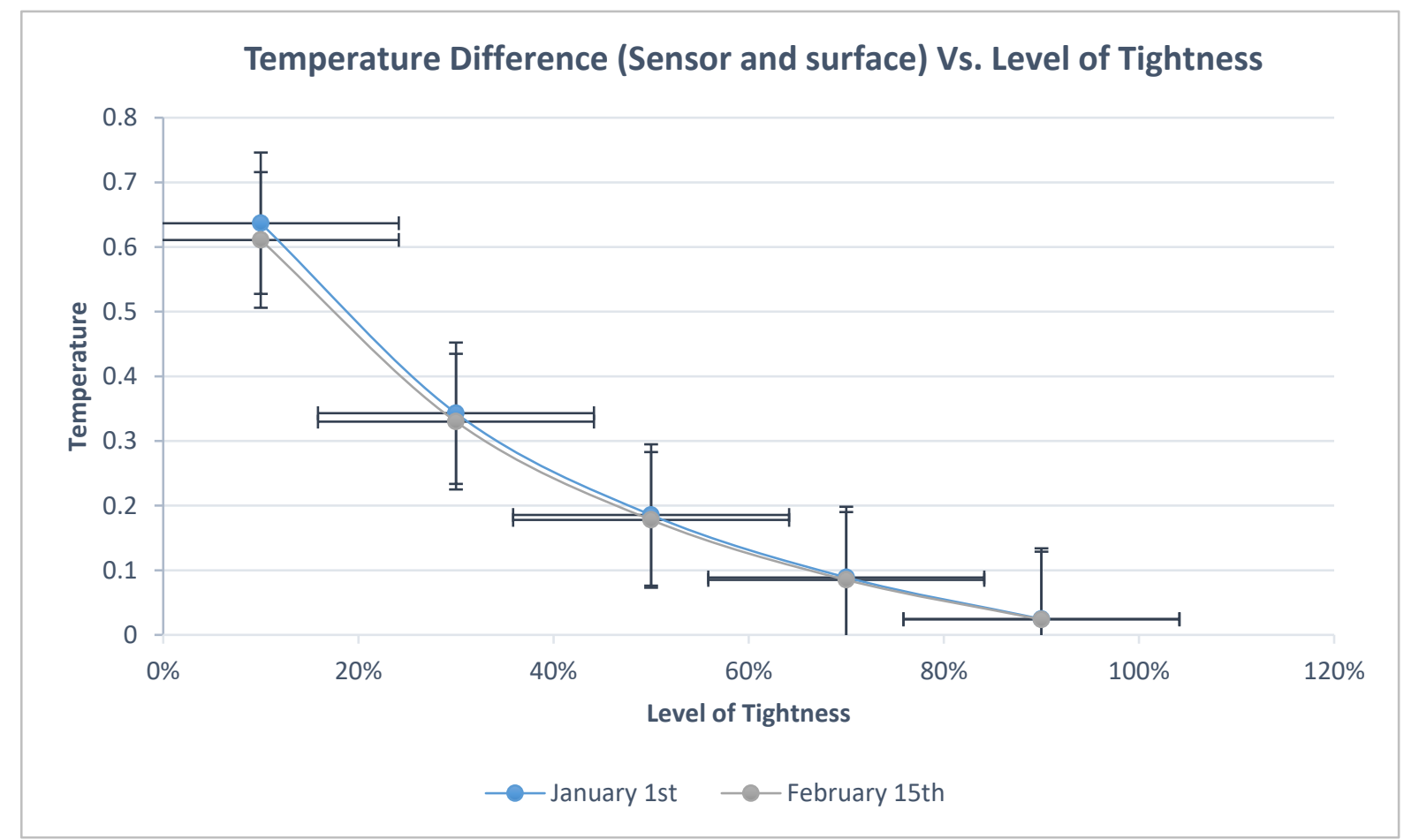

Figure 26- Temperature difference between sensor and surface, Jan. $1^{\text {st }}$ Vs. Feb. $15^{\text {th }}$ 


\subsection{April 1 $1^{\text {st }- \text { Holiday }}$}

The third set of simulation done in April $1^{\text {st }}$ with 45 days from second simulation.

Originally the April $1^{\text {st }}$ was set as a Holiday. Based on model's setting there is no occupancy in the room during holidays and the heating radiator system is working at the minimum during the day. Therefore, there is no warming up period, occupancy hours and the graph moves very smooth during the whole 24 hours. However still graphs follow the same rules as two other dates. With increasing the levels of tightness, the blue lines move toward the red line. Figure 27 to 31 are showing this procedure.

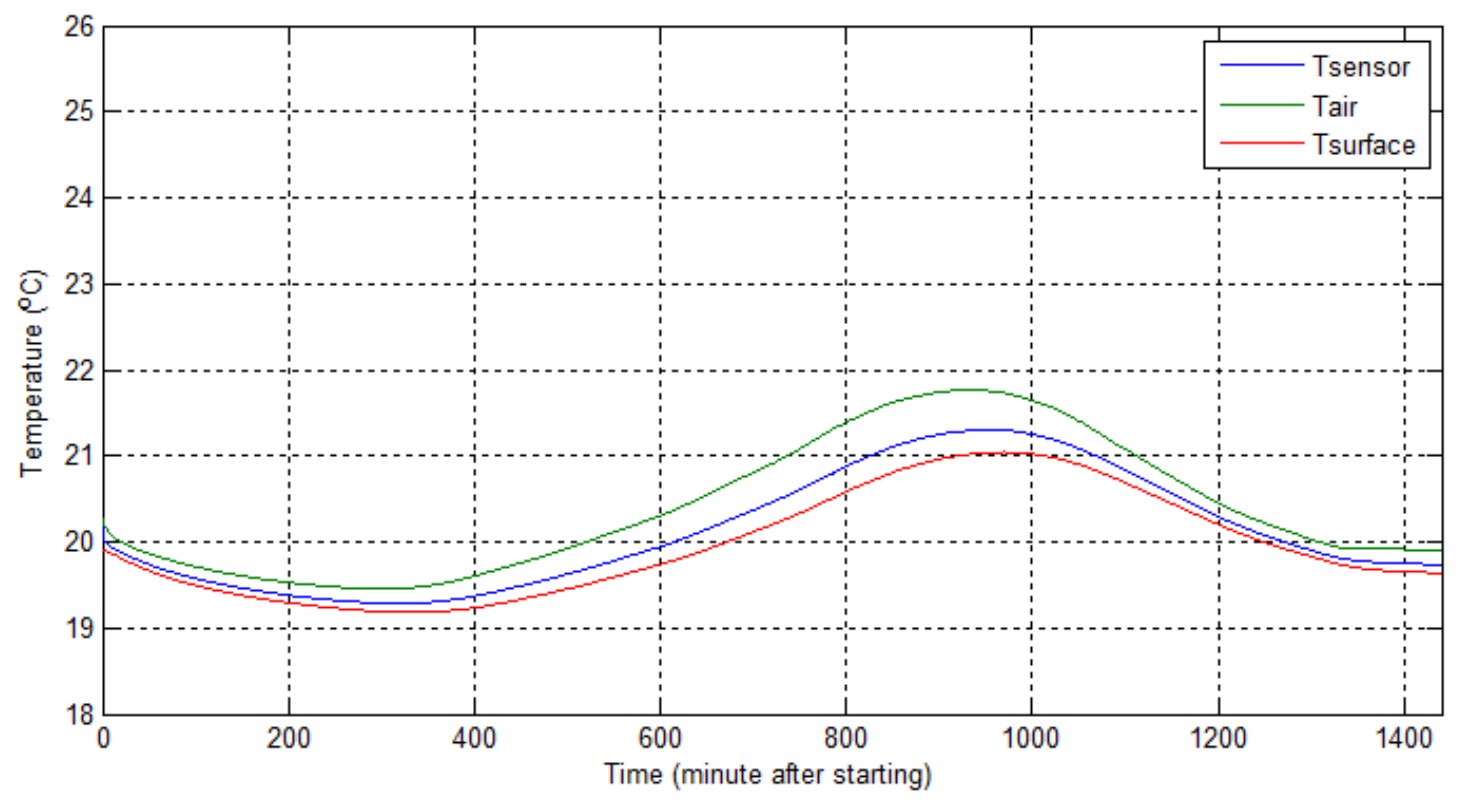

Figure 27- loose, April 1st (Holiday) 


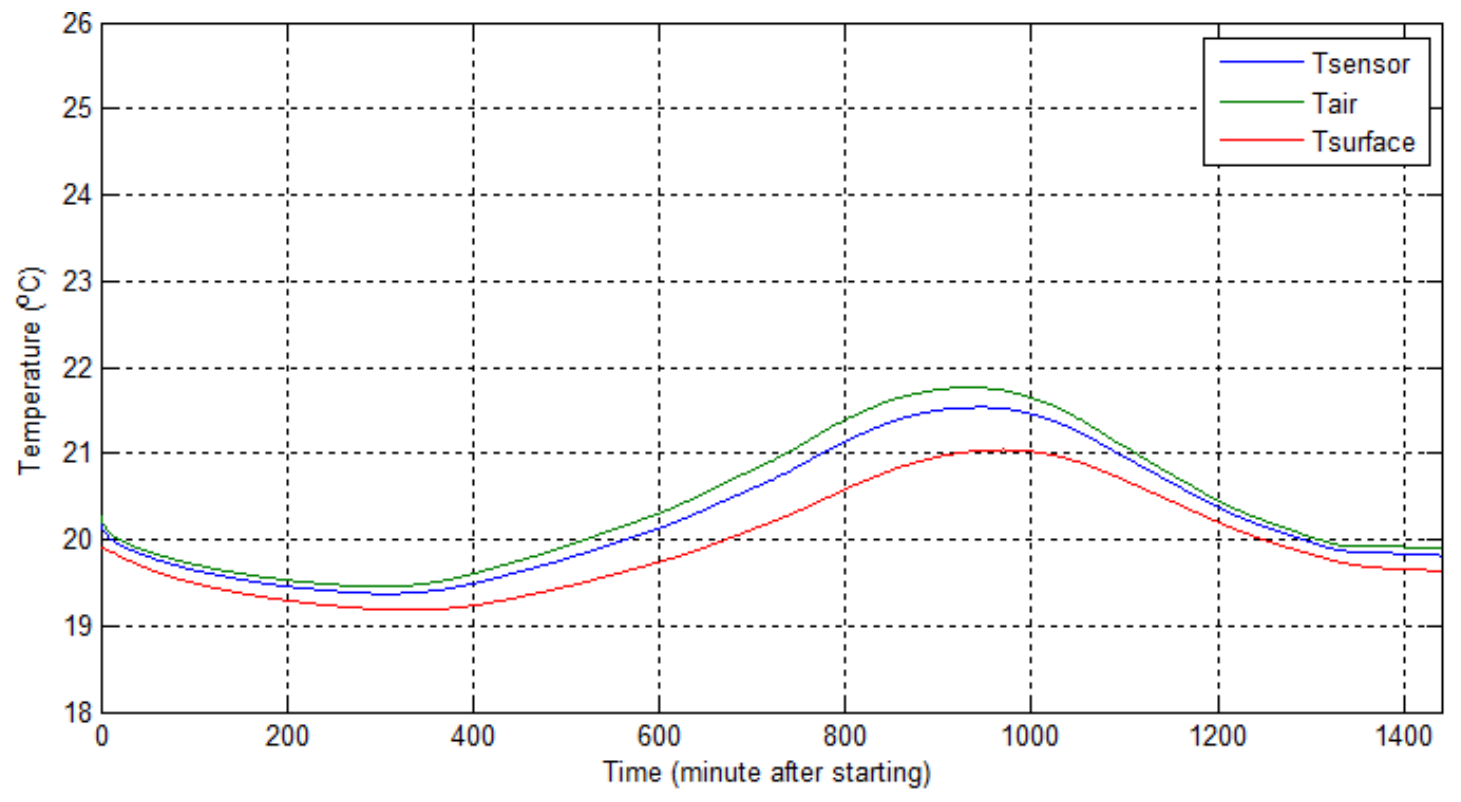

Figure 28- Very Loose, April 1st (Holiday)

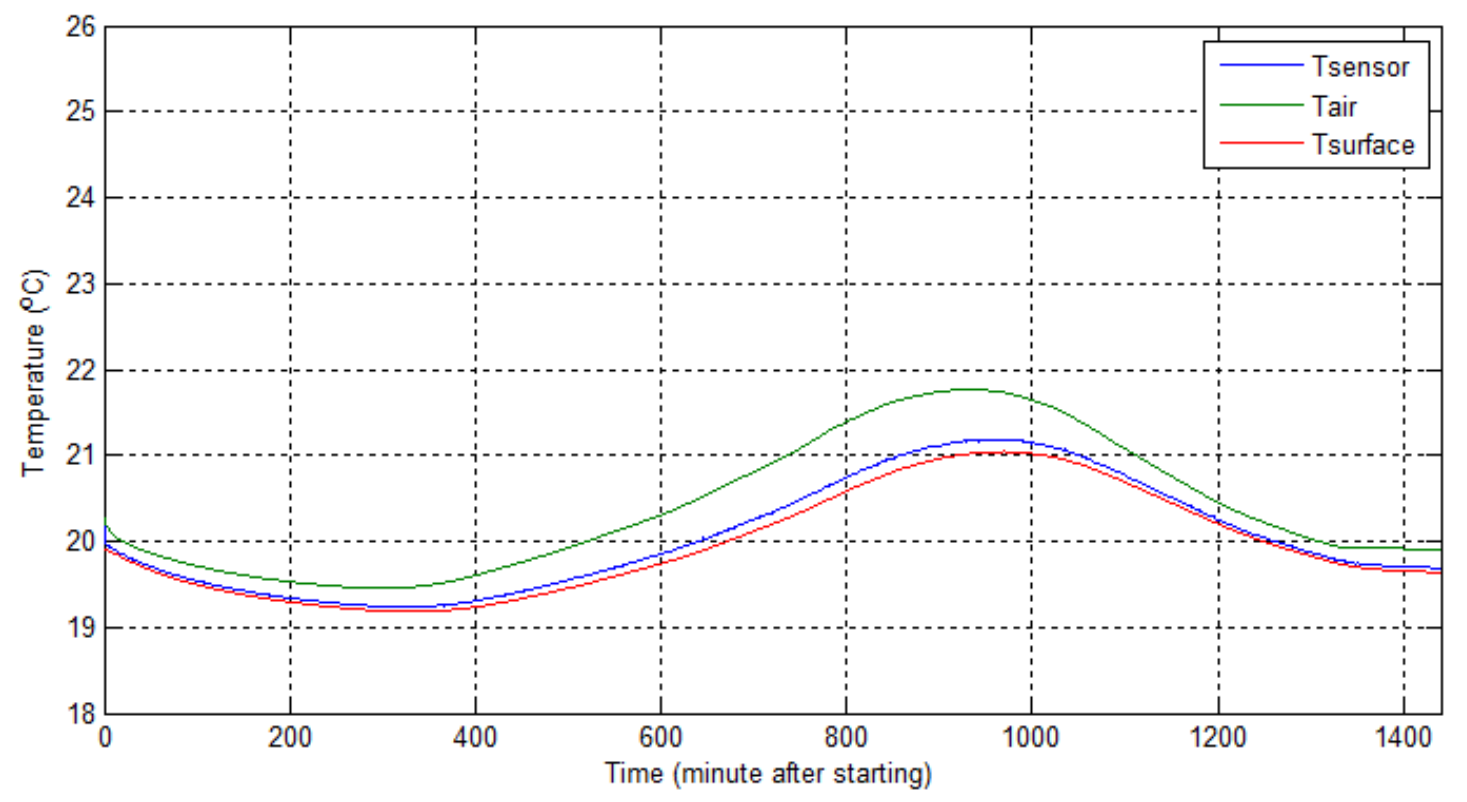

Figure 29- Medium, April 1st (Holiday) 


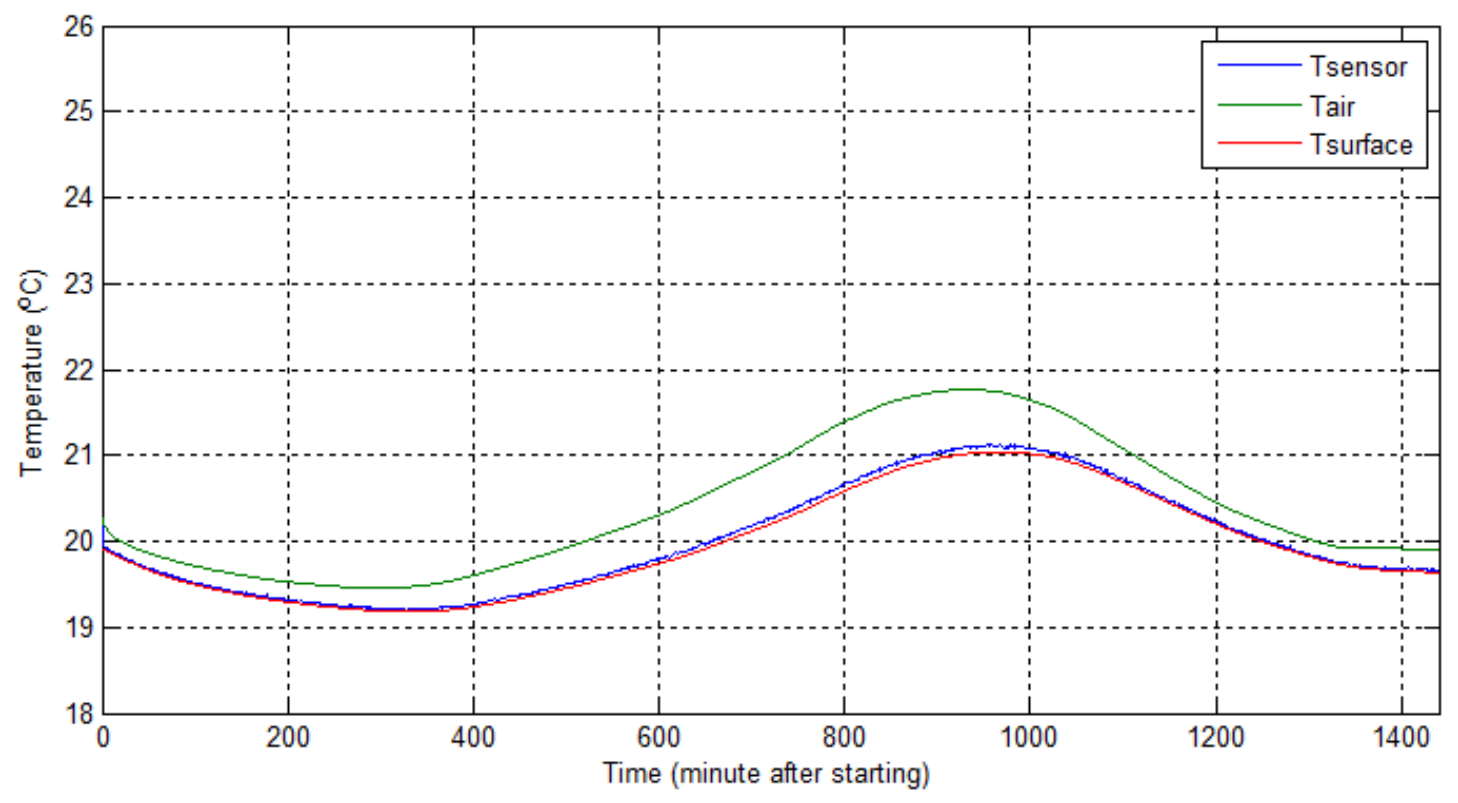

Figure 30- Tight, April 1st (Holiday)

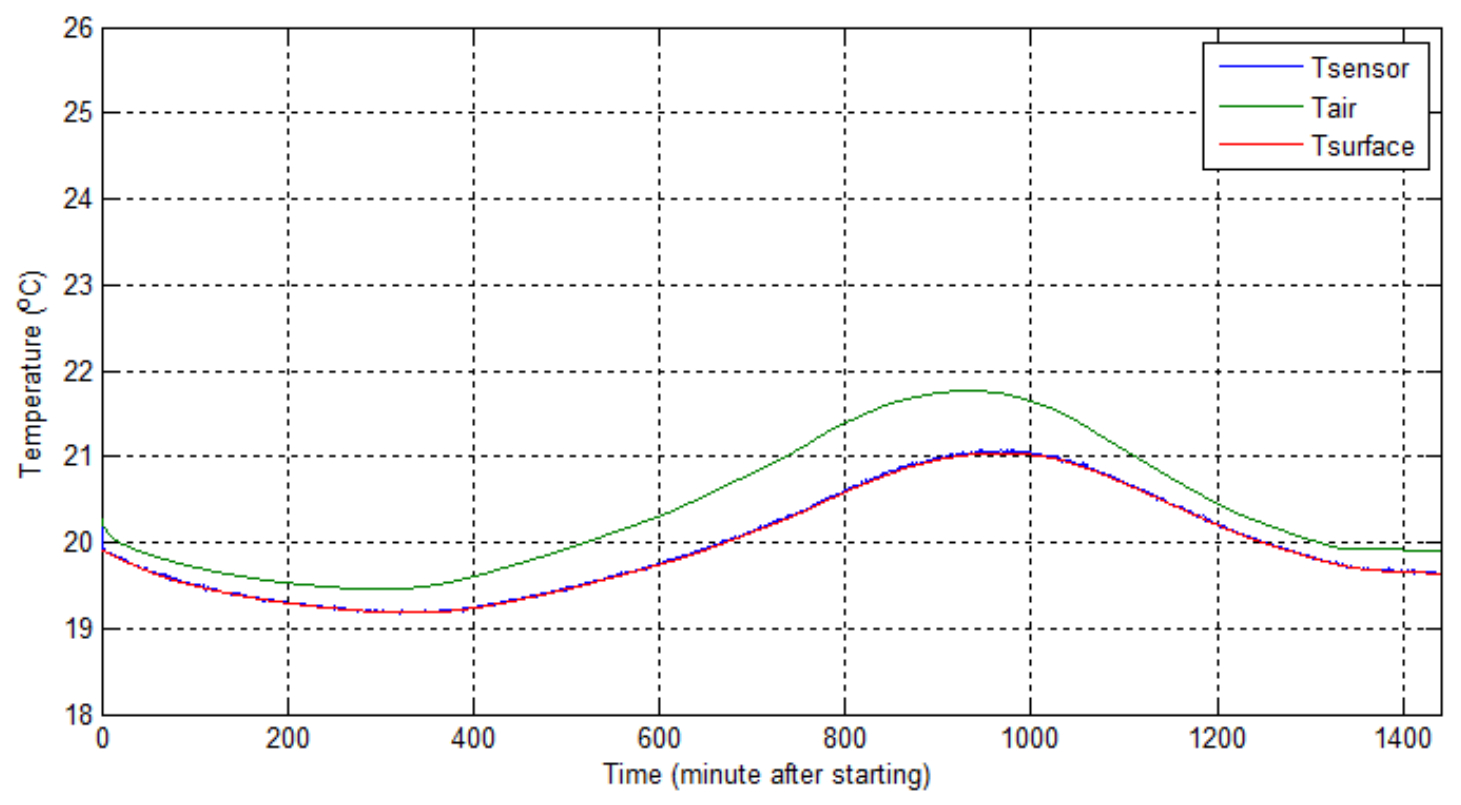

Figure 31-Very tight, April 1st (Holiday) 
The results on table 9 follow the same procedure as pervious simulation. The more tightness between sensor and wall surface, the higher sensor accuracy. However, the amount of sensor accuracy increased by 50 percent compare to two other dates (Figure 32). This 50 percent improvement is a result of smaller temperature difference between wall surface temperature and indoor air temperature. As the heating radiator works at minimum the indoor air temperature and wall surface temperature is getting very similar therefore the data generated by sensor is very close to wall surface temperature.

\begin{tabular}{|c|c|c|c|c|}
\hline \multirow{7}{*}{ 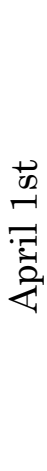 } & $\begin{array}{l}\text { Contact } \\
\text { level }\end{array}$ & $\begin{array}{l}\text { Contact } \\
\text { percentage }\end{array}$ & $\begin{array}{l}\text { Temp. difference between } \\
\text { sensor and air }{ }^{\circ} \mathrm{C}\end{array}$ & $\begin{array}{l}\text { Temp. difference between } \\
\text { sensor and surface }{ }^{\circ} \mathrm{C}\end{array}$ \\
\hline & & & $\sqrt{\frac{1}{N} \sum_{i=1}^{N}\left[T_{\text {Sensor }}-T_{A i r}\right]^{2}}$ & $\sqrt{\frac{1}{N} \sum_{i=1}^{N}\left[T_{\text {Sensor }}-T_{\text {Surface }}\right]^{2}}$ \\
\hline & Very Loose & 90 & 0.14 & 0.32 \\
\hline & Loose & 70 & 0.29 & 0.17 \\
\hline & Medium & 50 & 0.37 & 0.09 \\
\hline & Tight & 30 & 0.42 & 0.04 \\
\hline & Very Tight & 10 & 0.46 & 0.01 \\
\hline
\end{tabular}

Table 9 


\subsection{April 1st-Regular}

The simulation was repeated for April $1^{\text {st }}$ with changing the occupancy schedule to regular day instead of holiday. In this situation as graphs are showing, the results are very similar to January $1^{\text {st }}$ and February $15^{\text {th }}$. Also, table 10 is showing similar results as two other dates.

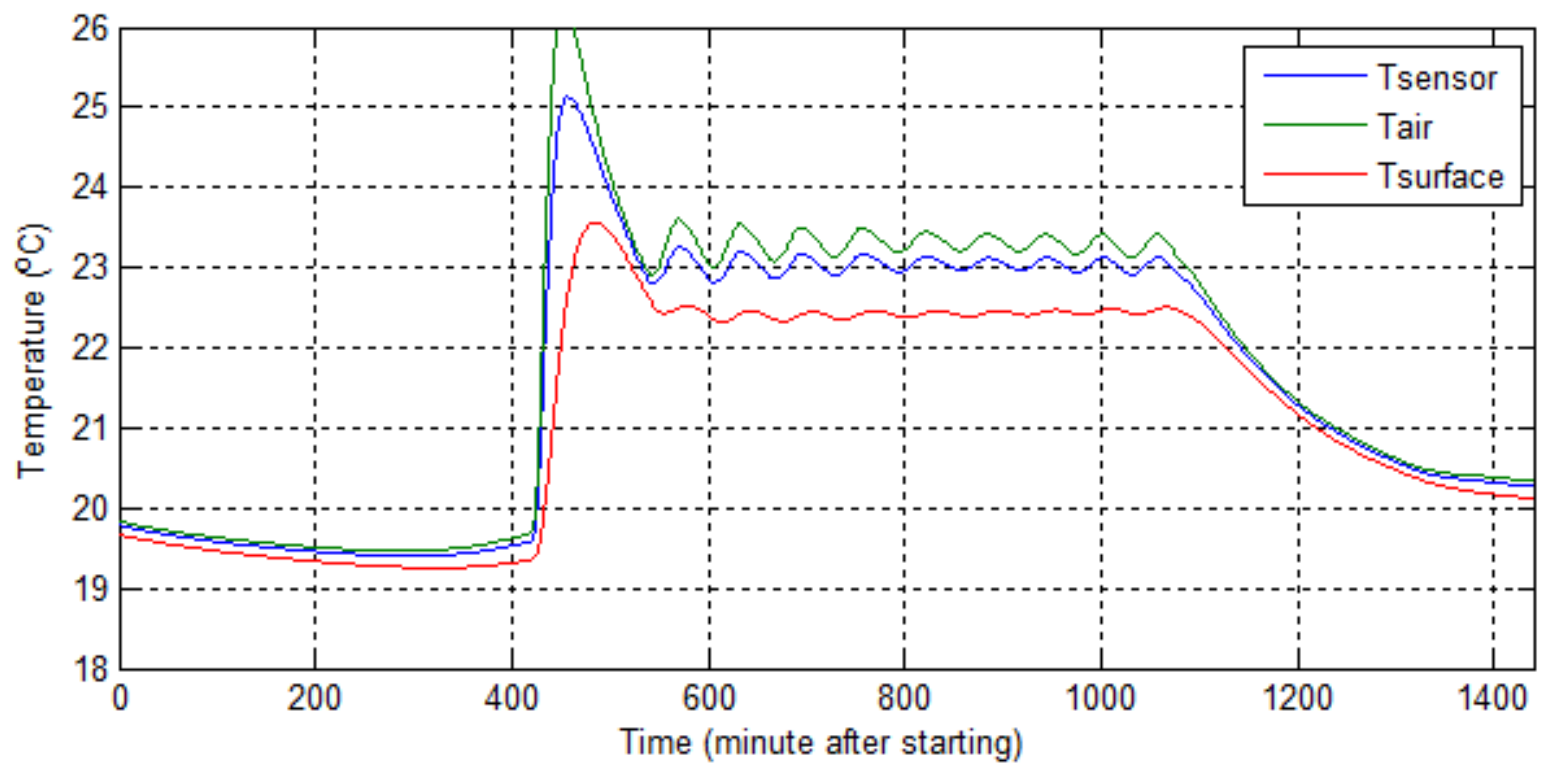

Figure 32-Very loose, April $1^{\text {st }}$ (Regular)

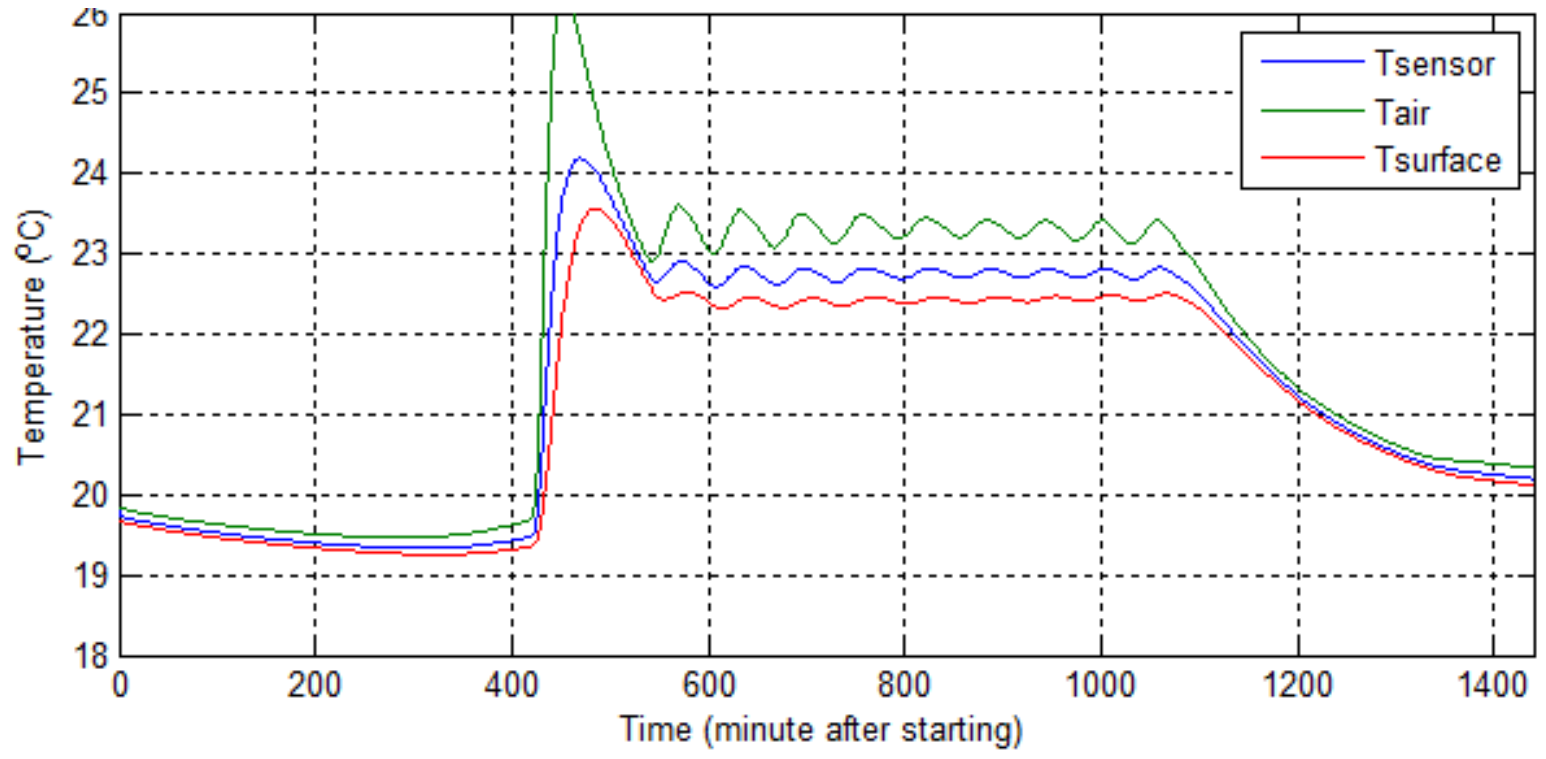

Figure 33- Loose, April $1^{\text {st }}$ (Regular) 


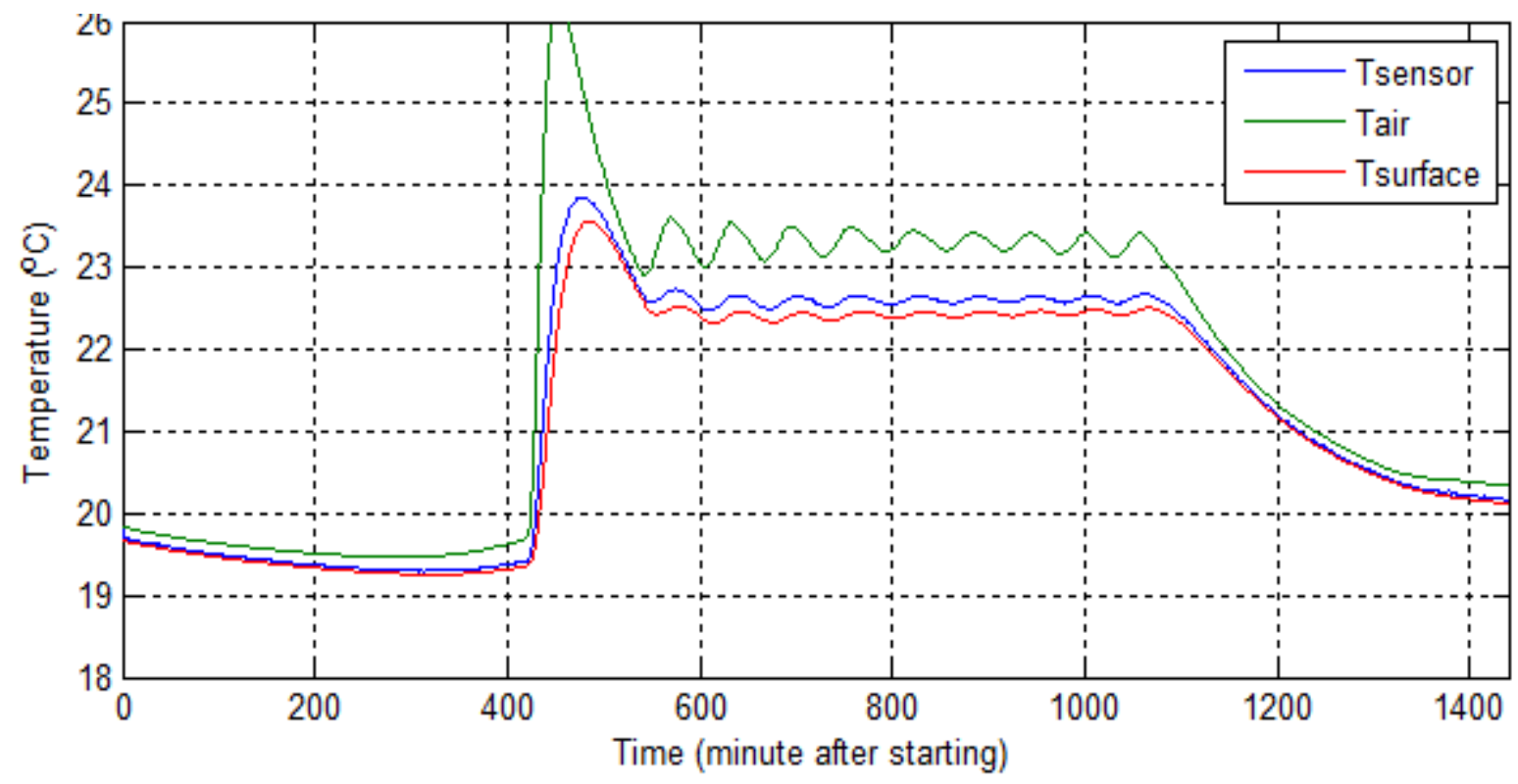

Figure 34- Medium, April $1^{\text {st }}$ (Regular)

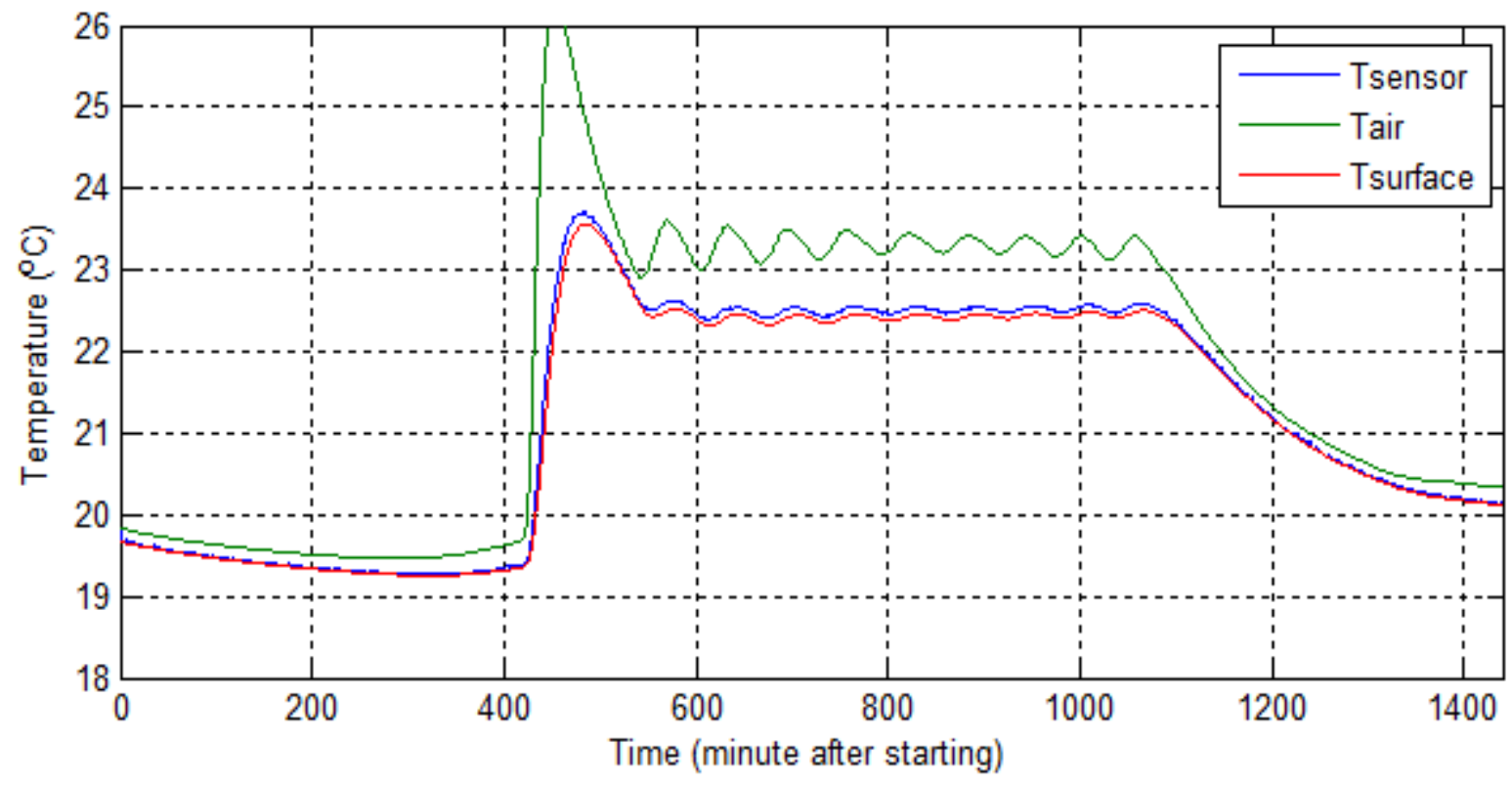

Figure 35-Tight, April $1^{\text {st }}$ (Regular) 


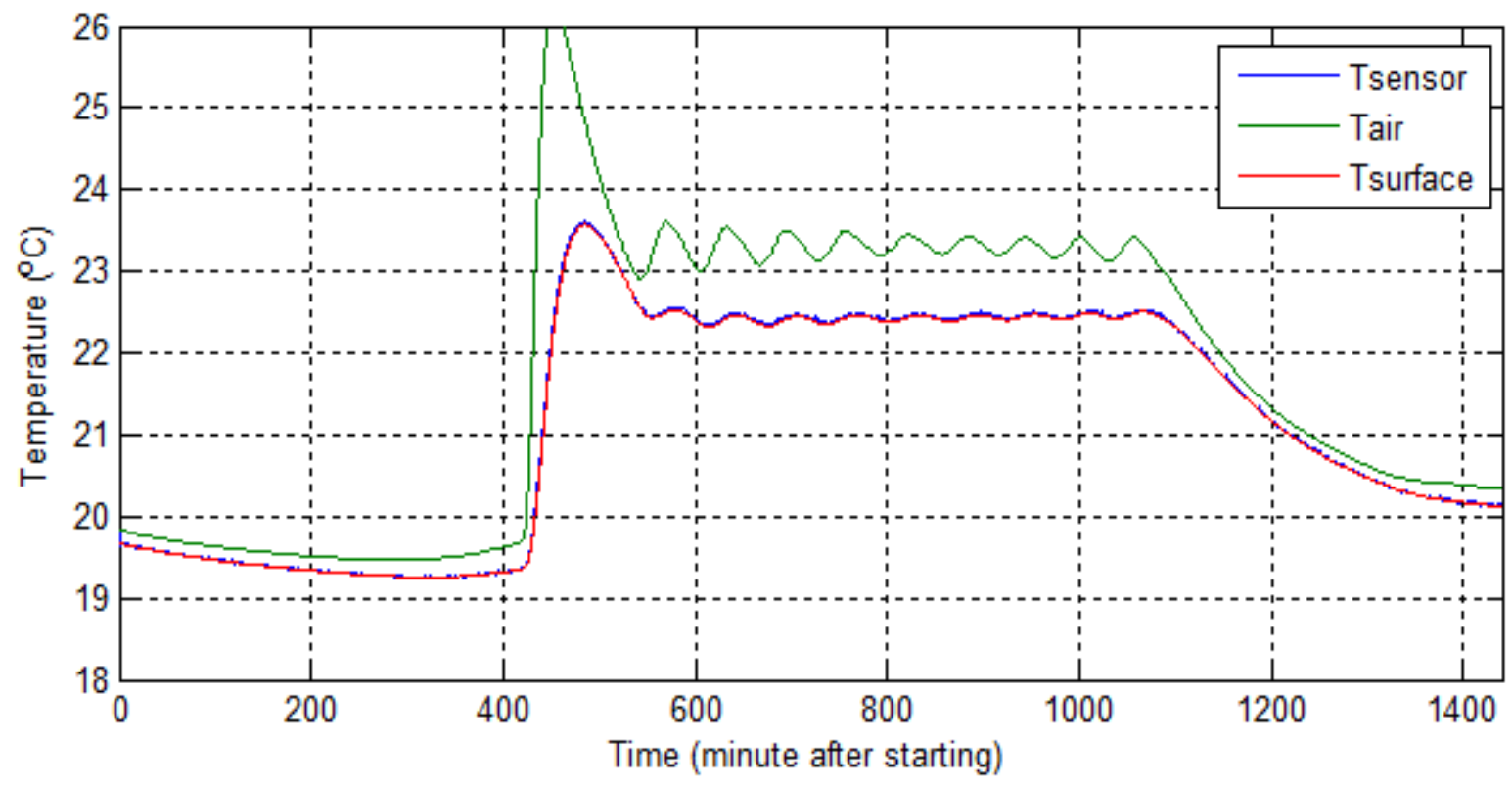

Figure 36- Very Tight, April $1^{\text {st }}$ (Regular)

\begin{tabular}{|c|c|c|c|c|}
\hline \multirow{7}{*}{ 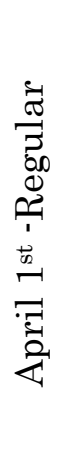 } & $\begin{array}{l}\text { Contact } \\
\text { level }\end{array}$ & $\begin{array}{l}\text { Contact } \\
\text { percentage }\end{array}$ & $\begin{array}{l}\text { Temp. difference between } \\
\text { sensor and air }{ }^{\circ} \mathrm{C}\end{array}$ & $\begin{array}{l}\text { Temp. difference between } \\
\text { sensor and surface }{ }^{\circ} \mathrm{C}\end{array}$ \\
\hline & & & $\sqrt{\frac{1}{N} \sum_{i=1}^{N}\left[T_{\text {Sensor }}-T_{A i r}\right]^{2}}$ & $\sqrt{\frac{1}{N} \sum_{i=1}^{N}\left[T_{\text {Sensor }}-T_{\text {Surface }}\right]^{2}}$ \\
\hline & Very Loose & 10 & 0.27 & 0.59 \\
\hline & Loose & 30 & 0.55 & 0.31 \\
\hline & Medium & 50 & 0.69 & 0.17 \\
\hline & Tight & 70 & 0.78 & 0.08 \\
\hline & Very Tight & 90 & 0.85 & 0.02 \\
\hline
\end{tabular}

Table 10

The figure 37 illustrates all four scenarios at once. As the graph is showing, January $1^{\text {st }}$, February $15^{\text {th }}$ and April $1^{\text {st }}$ (Regular) are located in a same range while there is a big gap between April 1 $1^{\text {st }}$ (Holiday) and rest of the scenarios. This gap indicates the absence of heating radiator. This situation resembles warmer month of the year such as summer time which the heating radiator is off.

When there is no heating radiator or it works at minimum, the temperature difference between indoor room and wall surface will be much smaller therefore the impact of ambient 
air on sensor accuracy will decrease. If a complete simulation done for 12 months of a year, probably all warmer months will remain close to April $1^{\text {st }}$ (holiday) while colder months will be placed close to January $1^{\text {st }}$ and February $15^{\text {th }}$.

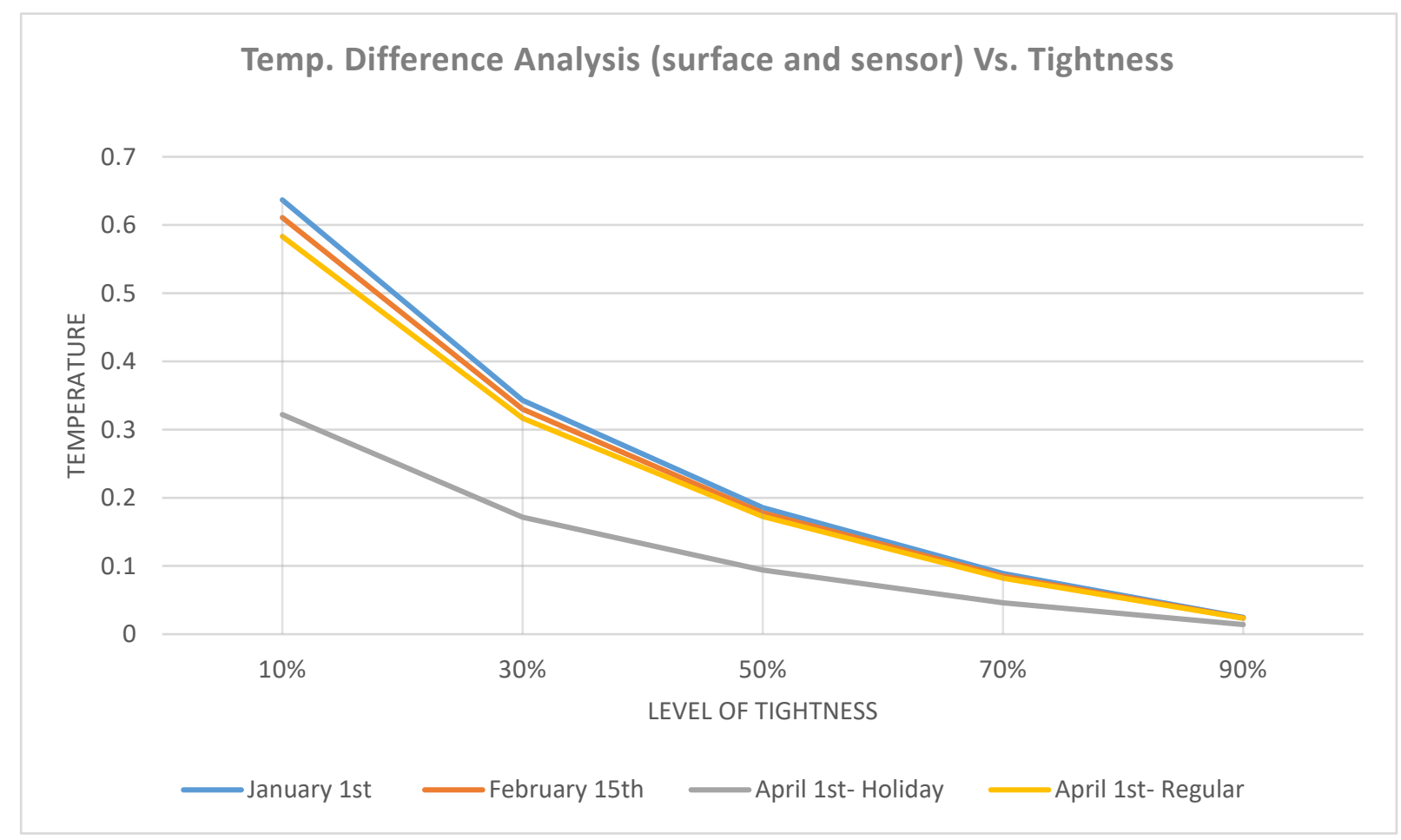

Figure $37-$ Comparing Jan. $1^{\text {st }}$, Feb. $15^{\text {th }}$, April $1^{\text {st }}$ Holiday and April $1^{\text {st }}$ Regular

Also, this study emphasis on the importance of wall assembly performance. The higher the performance, the lower heat conduction through the envelope and finally more representative data. The high-performance wall assembly eliminates thermal bridges and heat lost through the wall, as a result the wall surface temperature will remain close to ambient air temperature.

In addition, analyzing all sets of simulation show that there is always a time lag between the sensor performance and the surface temperature. Based on research done by Fraden (Fraden, 2010) if sensor couple to surface for very long time (infinite) it will reach 
equilibrium. later, he mentioned that although this theory can be correct but it is not realistic. Therefore, always time lag in heat transfer can generate error and inaccuracy in data representativeness.

Another issue is existence of thermal resistance even in the situation that sensor embedded into the object. Therefore, surface temperature and sensor's data never can be 100 percent equal and achieving 100 percent measurement representativeness is never possible.

\begin{tabular}{|l|l|l|l|l|}
\hline \multirow{2}{*}{ Contact Level } & \multicolumn{4}{|c|}{ Temperature difference between the sensor and the surface } \\
\cline { 2 - 5 } & January 1 & February 15 & $\begin{array}{l}\text { April 1 } \\
\text { st }\end{array}$ & $\begin{array}{l}\text { April 1st } \\
\text { (Hogular) }\end{array}$ \\
\hline $10 \%$ & 0.63 & 0.61 & 0.59 & 0.32 \\
\hline $30 \%$ & 0.34 & 0.32 & 0.31 & 0.17 \\
\hline $50 \%$ & 0.18 & 0.17 & 0.17 & 0.09 \\
\hline $70 \%$ & 0.08 & 0.08 & 0.08 & 0.04 \\
\hline $90 \%$ & 0.02 & 0.02 & 0.02 & 0.01 \\
\hline
\end{tabular}




\section{Sensitivity Analyses}

Sensor is a very sensitive device that need a controlled environment to perform the best. The problem is that providing controlled environment is not always possible but there are methods that help to increase sensor accuracy and measurement representativeness. In designing a sensor several factors impact on the accuracy of system, shape/size and fabrication material are among thoes.

\subsection{Size:}

Many scientists studied the impact of sensor dimension on accuracy of the result. In a research conducted by Farden, he studied the design of sensor when it is much smaller than measurement object in this situation the object will act as infinite heat source. In fact, as the object gets larger or sensor becomes smaller the sensor shows more accurate surface temperature (Fraden, 2010). Based on this research, some dimensional modification was applied to the sensor. The original sensor was cylindrical shape $1 \mathrm{~mm}$ in radius and $5 \mathrm{~mm}$ in height. The new size is $1 \mathrm{~mm}$ radius to $1 \mathrm{~mm}$ height. Tables 12 and 13 are illustrating root mean square error (temperature difference between surface and sensor) before and after modification for two dates of January $1^{\text {st }}$ and April $1^{\text {st }}$ (Holiday). Images 39 and 40 are demonstrating these differences on graphs. 


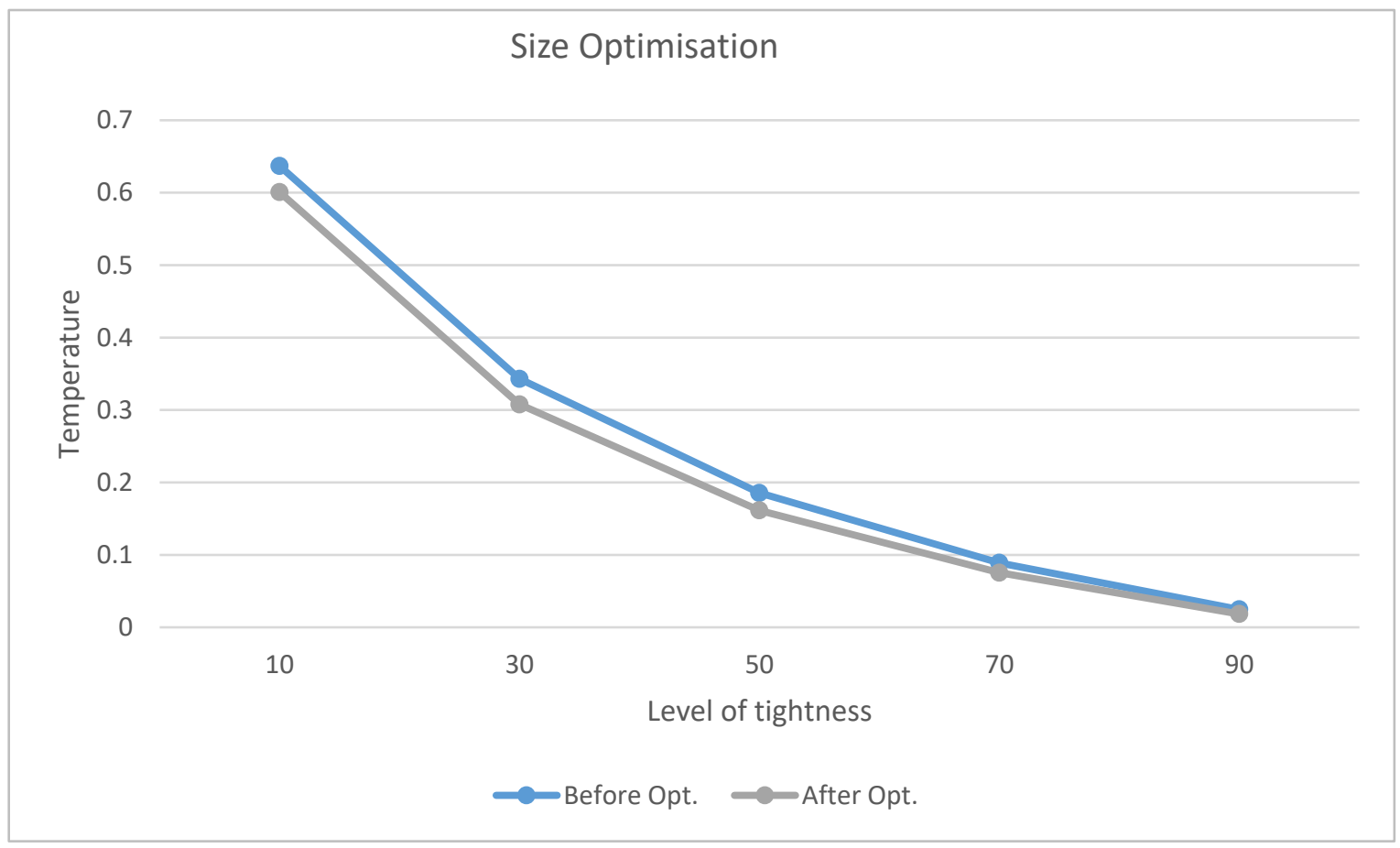

Figure 38- Size optimization, January 1st

\begin{tabular}{|c|c|c|c|c|c|c|}
\hline \multicolumn{7}{|c|}{ Size Modification } \\
\hline \multirow{8}{*}{ 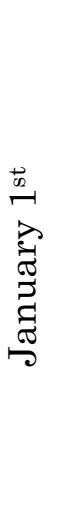 } & \multirow{4}{*}{$\begin{array}{l}\text { Contact } \\
\text { level } \\
\text { Very Loose }\end{array}$} & \multirow[t]{3}{*}{$\begin{array}{l}\text { Contact } \\
\%\end{array}$} & \multirow{2}{*}{\multicolumn{2}{|c|}{$\begin{array}{l}\text { Temp. difference between } \\
\text { sensor and air }{ }^{\circ} \mathrm{C} \\
\sqrt{\frac{1}{N} \sum_{i=1}^{N}\left[T_{\text {Sensor }}-T_{\text {Air }}\right]^{2}}\end{array}$}} & \multirow{2}{*}{\multicolumn{2}{|c|}{$\begin{array}{l}\text { Temp. difference between } \\
\text { sensor and surface }{ }^{\circ} \mathrm{C} \\
\sqrt{\frac{1}{N} \sum_{i=1}^{N}\left[T_{\text {Sensor }}-T_{\text {Surface }}\right]^{2}}\end{array}$}} \\
\hline & & & & & & \\
\hline & & & Before Opt. & After Opt. & Before Opt. & After Opt. \\
\hline & & 90 & 0.29 & 0.28 & 0.63 & 0.60 \\
\hline & Loose & 70 & 0.59 & 0.56 & 0.34 & 0.30 \\
\hline & Medium & 50 & 0.75 & 0.71 & 0.18 & 0.16 \\
\hline & Tight & 30 & 0.84 & 0.80 & 0.08 & 0.07 \\
\hline & Very Tight & 10 & 0.90 & 0.86 & 0.02 & 0.01 \\
\hline
\end{tabular}

Table 12 


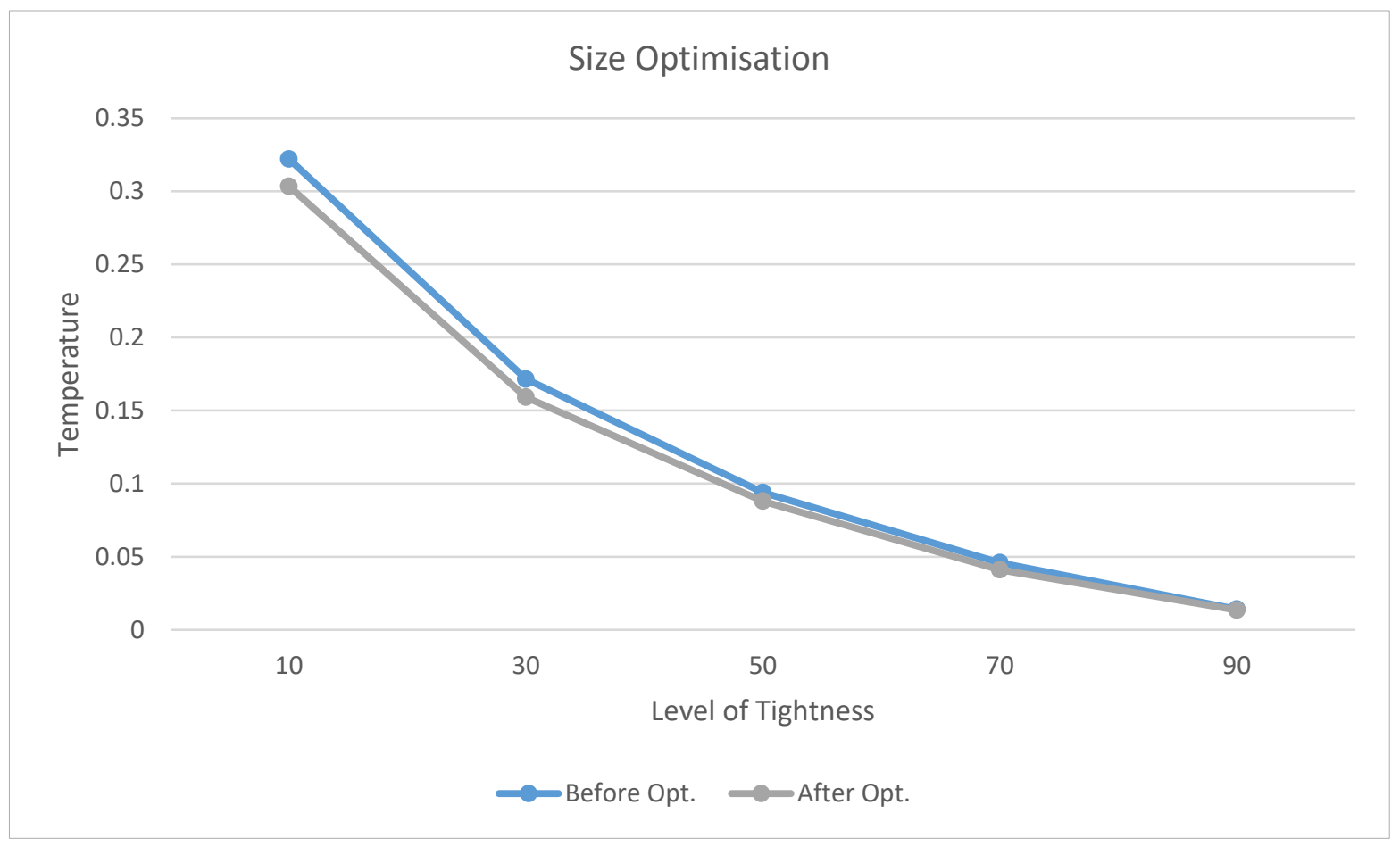

Figure 39- Size optimization, April 1st (Holiday)

\begin{tabular}{|c|c|c|c|c|c|c|}
\hline \multicolumn{7}{|c|}{ Size Modification } \\
\hline \multirow{8}{*}{$\begin{array}{l}\frac{1}{02} \\
\frac{2}{2} \\
\frac{2}{2} \\
\frac{2}{4}\end{array}$} & $\begin{array}{l}\text { Contact } \\
\text { level }\end{array}$ & $\begin{array}{l}\text { Contact } \\
\%\end{array}$ & \multirow{2}{*}{\multicolumn{2}{|c|}{$\begin{array}{l}\text { Temp. difference between } \\
\text { sensor and air }{ }^{\circ} \mathrm{C} \\
\sqrt{\frac{1}{N} \sum_{i=1}^{N}\left[T_{\text {Sensor }}-T_{\text {Air }}\right]^{2}}\end{array}$}} & \multirow{2}{*}{\multicolumn{2}{|c|}{$\begin{array}{l}\text { Temp. difference between } \\
\text { sensor and surface }{ }^{\circ} \mathrm{C} \\
\sqrt{\frac{1}{N} \sum_{i=1}^{N}\left[T_{\text {Sensior }}-T_{\text {Surface }}\right]^{2}}\end{array}$}} \\
\hline & & & & & & \\
\hline & & & Before Opt. & After Opt & Before Opt. & After Opt. \\
\hline & Very Loose & 90 & 0.14 & 0.14 & 0.32 & 0.30 \\
\hline & Loose & 70 & 0.29 & 0.29 & 0.17 & 0.16 \\
\hline & Medium & 50 & 0.37 & 0.37 & 0.09 & 0.08 \\
\hline & Tight & 30 & 0.42 & 0.42 & 0.04 & 0.04 \\
\hline & Very Tight & 10 & 0.46 & 0.46 & 0.01 & 0.01 \\
\hline
\end{tabular}

Table 13

The results of both simulation are showing that as the sensor gets smaller the accuracy increase. Specially at the weaker tightness level (10\%) the reduction is more visible. As the level of tightness increase, the root mean square error gets much smaller. In April $1^{\text {st, }}$ the optimization worked the best as the indoor air temperature and surface temperature were close and the sensor was much smaller. 


\subsection{Material}

Material selection is an important part of sensor fabrication. Appropriate type of metal increase thermal conductivity and sensitivity of sensor and finally impacts on the measurement representativeness. As mentioned earlier Platinum, Copper and Nickle are among the most popular metal in this field. Metals such as silver and gold also can be a good candidate as both have high conductivity. The drawback of these two metals are high price and rareness. Originally, the selected material for the sensor fabrication in this project was Copper while for the purpose of optimization silver was chosen. Silver has the highest thermal conductivity among other metals with specific heat capacity of $0.240 \mathrm{~J} / \mathrm{g} .{ }^{\circ} \mathrm{c}$ and density of $10.49 * 10^{-6} \mathrm{~g} / \mathrm{mm} 3$. The following tables are showing RMSE measurement before and after optimization for two date of January $1^{\text {st }}$ and April $1^{\text {st. }}$.

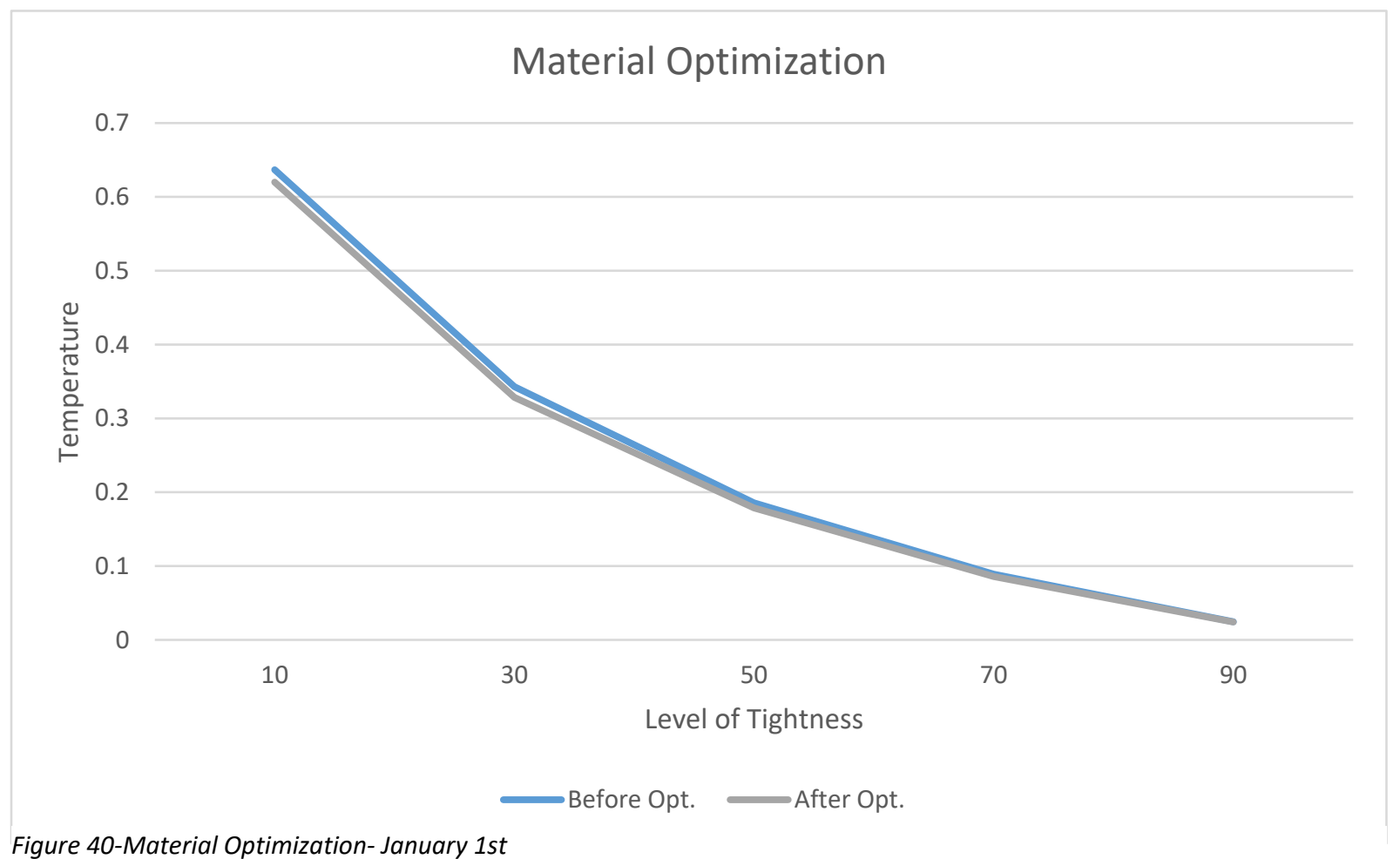




\begin{tabular}{|c|c|c|c|c|c|c|}
\hline \multicolumn{7}{|c|}{ Material Sensitivity } \\
\hline \multirow{8}{*}{ 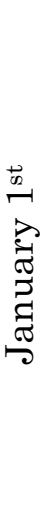 } & $\begin{array}{l}\text { Contact } \\
\text { level }\end{array}$ & $\begin{array}{l}\text { Contact } \\
\%\end{array}$ & \multirow{2}{*}{\multicolumn{2}{|c|}{$\begin{array}{l}\text { Temp. difference between } \\
\text { sensor and air }{ }^{\circ} \mathrm{C} \\
\sqrt{\frac{1}{N} \sum_{i=1}^{N}\left[T_{\text {Sensor }}-T_{\text {Air }}\right]^{2}}\end{array}$}} & \multirow{2}{*}{\multicolumn{2}{|c|}{$\begin{array}{l}\text { Temp. difference between } \\
\text { sensor and surface }{ }^{\circ} \mathrm{C} \\
\sqrt{\frac{1}{N} \sum_{i=1}^{N}\left[T_{\text {Sensor }}-T_{\text {Surface }}\right]^{2}}\end{array}$}} \\
\hline & & & & & & \\
\hline & & & Before Opt. & After Opt. & Before Opt. & After Opt. \\
\hline & Very Loose & 90 & 0.29 & 0.28 & 0.63 & 0.62 \\
\hline & Loose & 70 & 0.59 & 0.56 & 0.34 & 0.32 \\
\hline & Medium & 50 & 0.75 & 0.72 & 0.18 & 0.17 \\
\hline & Tight & 30 & 0.84 & 0.80 & 0.08 & 0.08 \\
\hline & Very Tight & 10 & 0.90 & 0.87 & 0.02 & 0.02 \\
\hline
\end{tabular}

Table 14

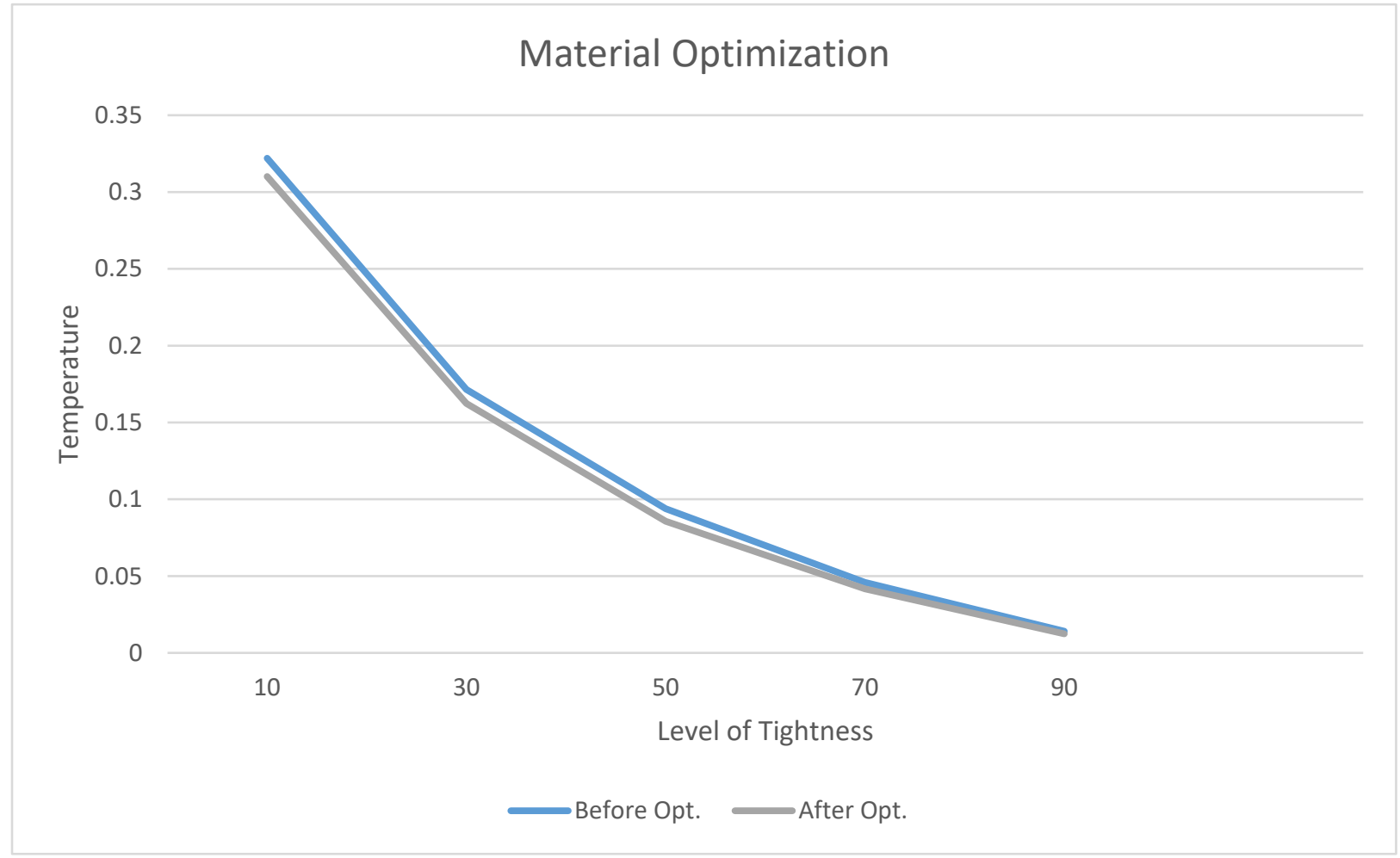

Figure 41- Material Optimization- April 1st (Holiday) 


\begin{tabular}{|c|c|c|c|c|c|c|}
\hline \multicolumn{7}{|c|}{ Material Sensitivity } \\
\hline \multirow{8}{*}{ 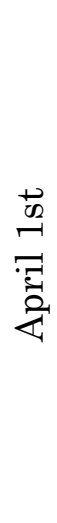 } & \multirow{3}{*}{$\begin{array}{l}\text { Contact } \\
\text { level }\end{array}$} & \multirow{3}{*}{$\begin{array}{l}\text { Contact } \\
\%\end{array}$} & \multirow{2}{*}{\multicolumn{2}{|c|}{$\begin{array}{l}\text { Temp. difference between } \\
\text { sensor and air }{ }^{\circ} \mathrm{C} \\
\sqrt{\frac{1}{N} \sum_{i=1}^{N}\left[T_{\text {Sensor }}-T_{\text {Air }}\right]^{2}}\end{array}$}} & \multirow{2}{*}{\multicolumn{2}{|c|}{$\begin{array}{l}\text { Temp. difference between } \\
\text { sensor and surface }{ }^{\circ} \mathrm{C} \\
\sqrt{\frac{1}{N} \sum_{i=1}^{N}\left[T_{\text {Sensor }}-T_{\text {Surface }}\right]^{2}}\end{array}$}} \\
\hline & & & & & & \\
\hline & & & Before Opt. & After Opt. & Before Opt. & After Opt. \\
\hline & Very Loose & 90 & 0.14 & 0.14 & 0.32 & 0.31 \\
\hline & Loose & 70 & 0.29 & 0.29 & 0.17 & 0.16 \\
\hline & Medium & 50 & 0.37 & 0.37 & 0.09 & 0.09 \\
\hline & Tight & 30 & 0.42 & 0.42 & 0.04 & 0.04 \\
\hline & Very Tight & 10 & 0.46 & 0.46 & 0.01 & 0.01 \\
\hline
\end{tabular}

Table 15

As it is clear on both graphs (Figure $40 \& 41$ ), by replacing copper to silver the accuracy of sensors slightly improved. Although this change is not very impressive but along other changes such as size, location etc., optimization will be more effective. When the coupling between sensor and wall is poor the optimization is more effective but as the contact level increase the temperature difference between wall surface and sensor (RMSE) before and after optimization does not change much. 


\section{Conclusion}

Different internal and external factors impact sensor's accuracy and measurement representativeness. Coupling is one of the most crucial factors. The more the sensor decouple from surrounding environment, the more representative data it will generate. one of the methods for decoupling the sensor from the environment is to embed it into the object. In this project $10 \%, 30 \%, 50 \%, 70 \%$ and $90 \%$ sensor's tightness levels were examined. The simulations were repeated for three different dates with different weather condition (very cold, cold and mild temperature). The length of simulation was set for 24 hours. The result of experiment for January $1^{\text {st }}$, February $15^{\text {th }}$ and April $1^{\text {st }}$ (regular) were very similar, with increasing the insertion depth, the temperature difference between sensor and surface (RMSE) decreased. The smaller RMSE the higher measurement accuracy. However, the result was different for April $1^{\text {st }}$ (holiday). Since the heating radiator was working at minimum during holidays, the temperature difference between the indoor room and wall surface (RMSE) drastically decreased. This reduction help the sensor to increase measurement accuracy (as the impact of ambient air on sensor decrease). Therefore, the result for April $1^{\text {st }}$ (holiday) was 50 percent more accurate than the other dates. This situation resembles warmer months of the year which there is no heating radiator and indoor air temperature and wall surface temperature are very similar (smaller $\Delta T)$.

Also, this study emphasises on importance of wall assembly quality. The high-performance wall assembly reduce thermal bridges and heat lost (conduction) through the wall. In this situation, the wall surface and indoor room temperature can reach to the equilibrium faster and the temperature difference becomes smaller. Therefore, sensor would generate more 
representative data. In addition, the smaller temperature difference between wall surface and ambient air provides satisfactory occupant thermal comfort.

Moreover, in this research project, sensor sensitivity was studied briefly and two optimization factors were introduced, materiality and size. The goal of this part of the project was to analyse the sensor's sensitivity after some design changes. Simulations were done for two days of January $1^{\text {st }}$ and April $1^{\text {st }}$ (Holiday).

Based on the Simulink results, reducing the size of the sensor has a direct relation with increasing measurement representativeness. However, the root mean square error (RMSE) results were not critically different after optimization. Also, the same result was achieved by material changes. In this simulation copper (thermal conductivity of $401 \mathrm{w} / \mathrm{m}^{*} \mathrm{k}$ ) was replaced with silver (thermal conductivity of $429 \mathrm{w} / \mathrm{m}^{*} \mathrm{k}$ ). Although silver is not a typical material in this field but it has higher conductivity than copper. The optimisation results indicate dimensional or material changes are not enough to reach the 100 percent accuracy. Therefore, it is suggested to apply different optimisation methods at the same time to reach higher levels of accuracy. 


\section{Further Research}

The sensorization is growing fast and many opportunities are existing in this field. The research completed up to this point is a respectable beginning in new subjects. The following research can be conducted on this topic:

- Same simulation study for cooling mode

- Doing same experiment based on physical model

- Comparing the simulation model with the physical model

- Exploring Impact of different wall assemblies on sensor accuracy. Like different type of insulation, material and wall thicknesses.

- Investigating impact of different shape, material and location on sensor's accuracy 


\section{Appendices}

Simulation details and screenshots

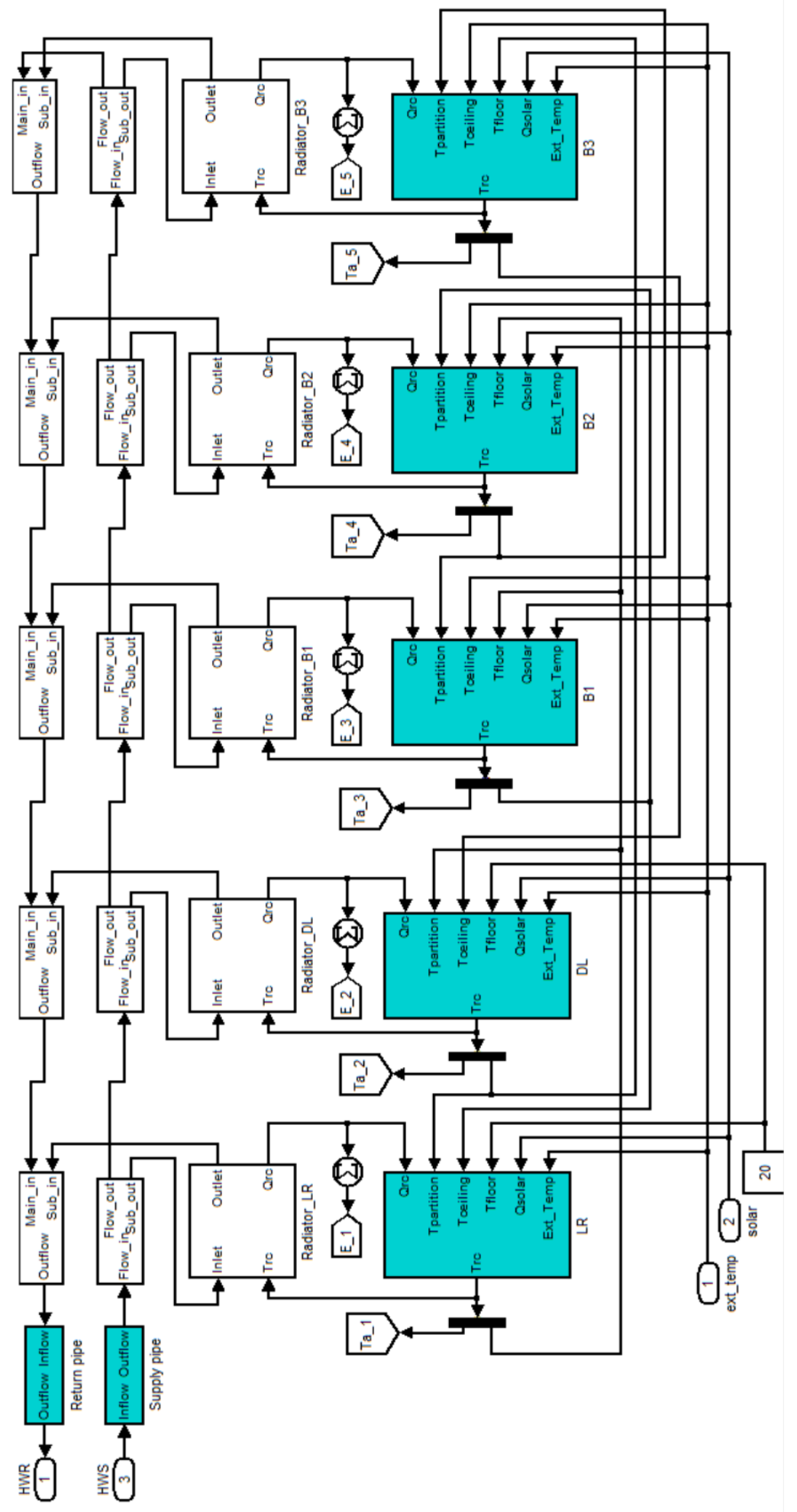

Image 5-Simulink model-3-bedroom house with living room and kitchen 

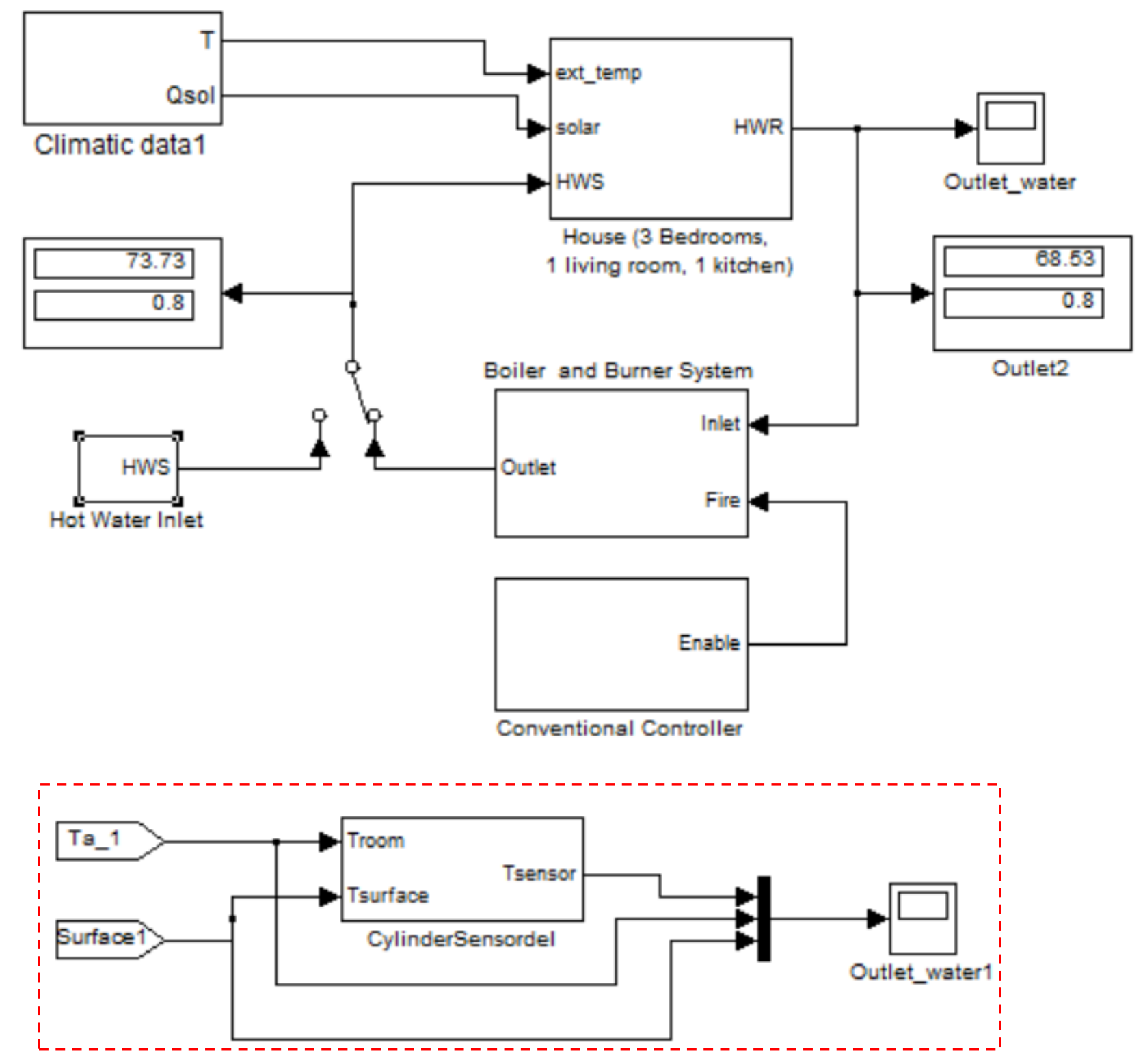

Simulink construction detail

Refer to appendices for higher quality image 


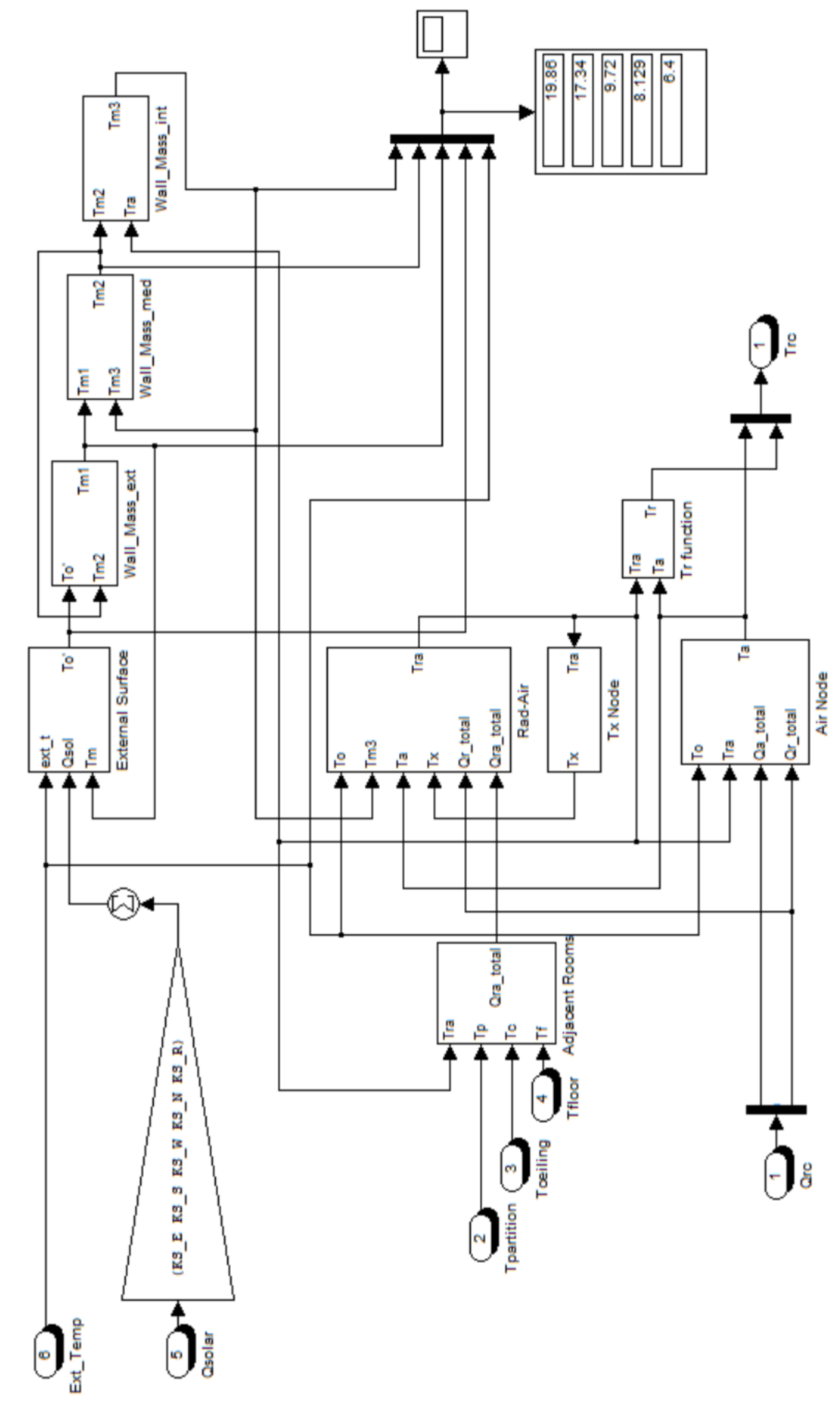

Image 6-Bedroom 1 construction 


\section{Bibliography}

Aggarwal, C. C. (2009). Managing and Mining Uncertain Data. Springer.

Aggarwal, C. C. (2013). Managing and Mining Sensor Data. New York: Springer.

Coy, E. B. (2010). Measurement of Transient Heat Flux and Surface Temperature Using Embedded Temperature Sensors. Journal of Thermophysics and Heat Transfer, 77-84.

Culbertson, D. P. (2001). United State of America Patent No. 6257758.

Emuwa, O. G. (2016). Impact of window walls on thermal comfort and energy efficiency in condominium buildings: an experimental study in Toronto. Toronto: Ryerson University.

Fraden, J. (2010). Handbook of Modern Sensors, Physics, Design and Application . San Diego: Springer Science.

Gershtein, A., \& Gershtein, S. (1996). Density of Silver Conversion . Retrieved from Convert me : http://www.convert-me.com/en/convert/density/denssilv.html

Hiraka, K., Shinagawa, R., Gogami, A., \& Luchi, T. (2008). Rapid-Response Hybrid-Type SurfaceTemperature Sensor. International Journal of Thermophysics, 1166-1173.

Jacob, M. (1969). Heat Transfer, Vol II. NewYork: Wiley.

Liao, Z., \& Huang, L. (2012). Physical-Rule based Adaptive Neuro-Fuzzy Inferential Sensor VS. GA-BP based Prediction Model in Indoor Temperature Predicting. Trans Tech Publications, 2179-2185.

Liao, Z., Jassar, S., \& Zhao, L. (2009). Adaptive neuro-fuzzy based inferential sensor model for estimating the average air temperature in space heating systems. Building and Enviroment, 1609-1616.

Makinwa, K., Pertijs, M., \& Meijer, G. (2014). Smart Sensor System. Delf: John Wiley \& Sons, Ltd.

Meijer, G. (2008). Smart sensor system. Delf, Netherland: A John Wiley and sons.

RAHARDJO, H., ZHANG, X. H., \& LEONG, E. C. (2012). Calibration of a thermal conductivity sensor for field measurement. Ge'otechnique, 81-85.

Ripka, P., \& Tipek, A. (2007). Modern Sensor Handbook. Chippenham, Wiltshire: ISTE.Ltd.

White, R. M. (1987, March). A sensor classification scheme. IEEE Trans Ultrason Ferroelectr Freq Control., pp. 124-126.

Wilson, J. S. (2005). Sensor Technology Handbook. Chandler, Arizona: Elsevier. 